\title{
Young onset dementia : characteristics and impact
}

Citation for published version (APA):

van Vliet, D. (2012). Young onset dementia : characteristics and impact. [Doctoral Thesis, Maastricht University]. Maastricht University. https://doi.org/10.26481/dis.20120920dv

Document status and date:

Published: 01/01/2012

DOI:

$10.26481 /$ dis.20120920dv

Document Version:

Publisher's PDF, also known as Version of record

\section{Please check the document version of this publication:}

- A submitted manuscript is the version of the article upon submission and before peer-review. There can be important differences between the submitted version and the official published version of record.

People interested in the research are advised to contact the author for the final version of the publication, or visit the DOI to the publisher's website.

- The final author version and the galley proof are versions of the publication after peer review.

- The final published version features the final layout of the paper including the volume, issue and page numbers.

Link to publication

\footnotetext{
General rights rights.

- You may freely distribute the URL identifying the publication in the public portal. please follow below link for the End User Agreement:

www.umlib.nl/taverne-license

Take down policy

If you believe that this document breaches copyright please contact us at:

repository@maastrichtuniversity.nl

providing details and we will investigate your claim.
}

Copyright and moral rights for the publications made accessible in the public portal are retained by the authors and/or other copyright owners and it is a condition of accessing publications that users recognise and abide by the legal requirements associated with these

- Users may download and print one copy of any publication from the public portal for the purpose of private study or research.

- You may not further distribute the material or use it for any profit-making activity or commercial gain

If the publication is distributed under the terms of Article $25 \mathrm{fa}$ of the Dutch Copyright Act, indicated by the "Taverne" license above, 


\section{Young onset dementia}

\section{Characteristics and impact}

Deliane van Vliet 


\section{Colophon}

Cover illustration: Helen van Vliet, www.helenvanvliet.nl Layout: typeset in $\mathrm{H}_{\mathrm{E}} \mathrm{X}$ by Pim Pullens

Print and production: Ridderprint, Ridderkerk

(C) Copyright D. van Vliet, Maastricht 2012

ISBN: $978-90-75579-56-7$ 


\section{Young onset dementia Characteristics and impact \\ PROEFSCHRIFT}

Ter verkrijging van de graad van doctor aan de Universiteit Maastricht, op gezag van de Rector Magnificus, Prof. dr. L.L.G. Soete volgens het besluit van het College van Decanen, in het openbaar te verdedigen op donderdag 20 september 2012 om 14:00 uur

door

Deliane van Vliet

geboren op 22 november 1981 te Oss 


\section{Promotores:}

Prof. dr. F.R.J. Verhey

Prof. dr. R.T.C.M. Koopmans (Radboud Universiteit Nijmegen)

\section{Copromotor:}

Dr. M.E. de Vugt

\section{Beoordelingscommissie:}

Prof. dr. R.W.H.M. Ponds (voorzitter)

Prof. dr. F. Pasquier (Université Lille)

Prof. dr. J.M.G.A. Schols

Dr. K. Schruers

Dr. S.U. Zuidema (Rijksuniversiteit Groningen)

The research described in this thesis was performed at the Department of Psychiatry and Neuropsychology, School for Mental Health and Neuroscience, Maastricht University, Maastricht, the Netherlands. The NeedYD-study was initiated by Maastricht University and carried out in collaboration with Radboud University Nijmegen and VU University Amsterdam. Partial funding for this study was provided by Florence Care Group in the Hague and the Dutch Alzheimer's Society.

Financial support for the publication of this thesis was kindly provided by Alzheimer Nederland, Internationale Stichting Alzheimer Onderzoek (ISAO), Lundbeck B.V., Novartis Pharma B.V., Nutricia Advanced Medical Nutrition, Roche Nederland B.V. 


\section{Contents}

1 General Introduction

2 Research protocol of the NeedYD-study (Needs in Young onset Dementia): a prospective cohort study on the needs and course of young-onset dementia

BMC geriatrics, 2010

3 Impact of young-onset dementia on caregivers: a review International Journal of Geriatric Psychiatry, 2010

4 Time to diagnosis in young-onset dementia as compared to lateonset dementia

Psychological Medicine, 2012

5 Caregivers' perspectives on the pre-diagnostic period in youngonset dementia International Psychogeriatrics, 2011

6 Prevalence of neuropsychiatric symptoms in young-onset compared to late-onset Alzheimer's disease

Dementia and Geriatric Cognitive Disorders, 2012.

7 Awareness and its association with affective symptoms in youngonset and late-onset Alzheimer's disease

Alzheimer's Disease \& Associated Disorders, 2012

8 General discussion

Summary

Samenvatting

Dankwoord

Curriculum Vitae

List of publications 

Chapter 1

\section{General Introduction}




\subsection{An introduction to young-onset dementia}

\section{Historical note}

On November $4^{\text {th }} 1906$, Alois Alzheimer gave a lecture that described a 51-year-old patient called Auguste Deter who presented with progressive cognitive impairment, focal symptoms, hallucinations, delusions and psychosocial incompetence. The postmortem examination found plaques, neurofibrillary tangles and arteriosclerotic changes. Emil Kraepelin separated this disease from senile dementia in 1910 and named it after Alzheimer ${ }^{1}$. Thus, the eponym Alzheimer, which now denotes the largest cause of primary degenerative dementias, was originally used to refer only to presenile dementia ${ }^{2}$.

Statement of Kraepelin in his Handbook of Psychiatry $(1910)^{2}$

'The clinical interpretation of this Alzheimer's Disease is still unclear. Although the anatomical findings suggest that we are dealing with a particularly serious form of senile dementia, the fact is that this disease sometimes starts as early as in the late forties.'

In 1969, there was an explosion of interest in Alzheimer's disease (AD) ${ }^{3}$ and clinicopathological studies showed that AD and senile dementia were part of the same disease spectrum ${ }^{4}$. Despite the increasing interest in dementia over the past forty years, investigators have paid relatively little attention to dementia with a younger onset. For this reason, the prevalence and impact of young-onset dementia have long been underestimated ${ }^{5,6}$. There is now increasing awareness that young-onset dementia is an important diagnostic category, not only because the biological mechanisms of dementia can be investigated without the confounding influence of age or vascular changes, but also because of its clinical relevance. Numerous authors have suggested the particular challenges that young-onset dementia patients and their families face ${ }^{7-9}$ and have advocated for specialized services for younger people with dementia. However, little is known regarding the clinical characteristics and impact of young-onset dementia on patients and their families and their specific needs. This information is essential to provide evidence-based guidelines for practice and to develop suitable health care services for this population.

\section{Definition}

Today, presenile dementia (or young- or early-onset dementia) is defined as dementia with an onset of symptoms before the age of 65 . Presenile dementia refers to the dementia types that commonly occur in presenescence, and young- or early-onset dementia refers to typical senile dementias that have an early onset. These terms have been used interchangeably, favoring the term young- or early-onset dementia over presenile dementia in recent years ${ }^{10}$. In this thesis, the term young-onset dementia (YOD) will be used, as suggested by the Young Onset Dementia Taskforce of the International Psychogeriatric Association in 2011. 
The age limit of 65 years is a fairly crude and arbitrary age threshold that is based on a sociological division in terms of retirement age and has no biological underpinnings ${ }^{11}$. While it is arguable whether such a non-scientific partition is justified, it is an important distinction from a practical point of view because of the different social and psychological dimensions of YOD and LOD and the possible implications for care practices ${ }^{12}$.

\section{Epidemiology}

The number of people suffering from dementia worldwide was estimated to be 27.7 million in $2003^{13}$, and the number is expected to rise in the following years up to 114 million by $2050^{14}$. The prevalence of YOD in the UK has been estimated to be 54 per 100.000 in the population between 30 and 65 years of age and 98 per 100.000 in the age group between 45 and $65^{15}$, with similar rates found in $\operatorname{Japan}^{16}$. No epidemiological studies of all YOD cases have been performed in the Netherlands. The prevalence of YOD in the Netherlands would be approximately 4500 in the 30-64 age group when applying the figures mentioned above on the Dutch population aged 30-65 according to Statistics Netherlands in 2011. However, another study in Finland showed a prevalence rate of 260 per 100.000 in the 30-64 age group for severe YOD, resulting in approximately 21.000 cases of YOD in the Netherlands. Therefore, the rates found by the other studies might be an underestimation. The large differences between studies may be partly due to different case-finding techniques. The incidence of primary dementia for the age group 45-64 years is estimated to be 12 cases per 100.000 persons per year ${ }^{17}$, which results in approximately 700 new cases of primary YOD per year in the Netherlands.

The prevalence rate range per 100.000 in the $45-65$ age group ${ }^{15,16,18}$ is between 15 and 35 for $\mathrm{AD}$ and between 8 to 39 for vascular dementia (VaD). AD, dementia with Lewy bodies (DLB) and VaD comprise a smaller proportion of cases in younger patients than in $\mathrm{LOD}^{11}$. In contrast, rare/hereditary causes, metabolic syndromes, treatable causes, frontotemporal dementia (FTD) and secondary dementias (e.g., due to alcohol abuse, HIV, Down syndrome or traumatic brain injury) are more common in YOD than in older people $\mathrm{e}^{5,19-21}$. The younger a person is, the higher the chance of a metabolic or genetic disease ${ }^{11}$. The prevalence of YOD is relatively low in comparison with LOD, but the absolute numbers are still substantial. Therefore, the needs and problems of people with YOD should not be overlooked.

\subsection{Specific aspects of young-onset dementia}

\section{The impact of young-onset dementia}

YOD can have devastating consequences because it afflicts people in their most productive years ${ }^{5}$. Younger people are commonly employed, and when the patient 


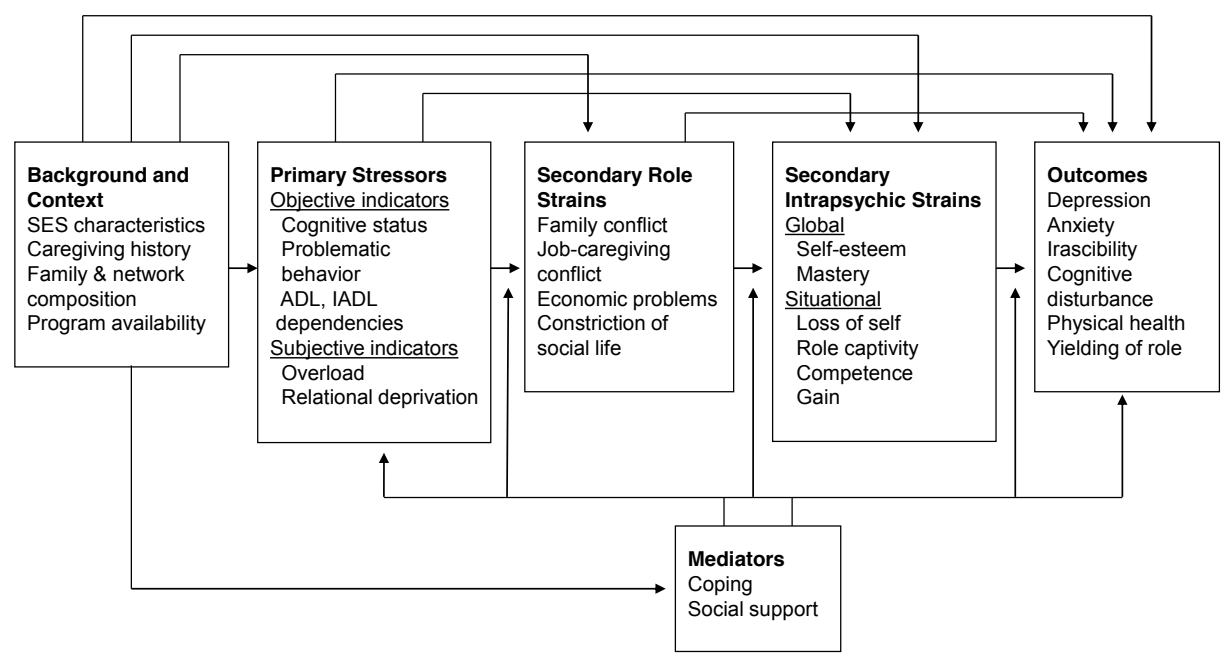

Figure 1.1: A conceptual model of Alzheimer's caregivers' stress. The stress process is made up of four domains: the background and context of stress; the stressors; the mediators of stress; and the outcomes or manifestations of stress. Adapted from Pearlin et. al. (1990).

is the primary source of income, a dementia diagnosis can lead to great financial strain. Other roles such as parenting and driving may also be curtailed, and the diagnosis may have a major impact in terms of the future plans of younger people. The impact may further increase because of the higher risk of genetic transmission $^{22,23}$.

Therefore, not only for YOD patients but also for their caregivers and their children, the disease may have a great emotional and social impact. It is well established that caring for a person with dementia has detrimental effects on caregiver psychological and physical health ${ }^{24,25}$. The possibility of an even more burdensome caregiving situation in YOD can be illustrated with a comprehensive model of the caregiver stress process proposed by Pearlin et al (1990) (figure 1.1). Four domains are identified: the background and context of stress, the stressors, the mediators and the outcomes of stress. Stressors can be either primary stressors, secondary role strains or secondary intrapsychic strains ${ }^{26}$. It is unknown how this model could apply to YOD caregivers, but the prevailing idea is that high secondary role strains and a lack of program availability are specific stressors in $\mathrm{YOD}^{8,27}$, leading to a negative caregiver outcome. In addition, primary stressors may differ because of the differences in etiologies between YOD and LOD. It is important to investigate the specific impact of YOD on patients' informal caregivers and whether this differs from the impact of LOD. This knowledge is a prerequisite to developing adequate support programs for YOD caregivers. 


\section{Diagnostic issues}

As yet, there is no curative or disease-modifying treatment for dementia. However, with the possibility of these treatments becoming available in the future, establishing a diagnosis in an early stage of the disease is of high importance. There is also increasing recognition that a timely dementia diagnosis is key to optimizing care and support benefits for patients and their caregivers.

However, distinguishing between normal ageing and the first symptoms of dementia can be a challenge ${ }^{28}$. The differential diagnosis of many dementia types may also present with difficulties because their clinical presentations overlap, and unique differentiating biomarkers are not yet available ${ }^{29}, 30$. Furthermore, patients and caregivers may be reluctant to seek help, and therapeutic nihilism may still lead physicians to not disclose a diagnosis ${ }^{31,32}$. Therefore, dementia is commonly under-diagnosed ${ }^{32}$, and the diagnosis is delayed until symptoms are well established $^{28}$. Numerous studies have aimed at improving the recognition and diagnosis of dementia by developing practice guidelines for primary care, improving screening tools for dementia or identifying neuropathological substrates of dementia $28,31,33-36$.

YOD may even be more complicated to detect and diagnose than LOD due to its lower a priori chance and the larger variety of etiologies ${ }^{5,11,19-21}$. In addition, many of the YOD types are characterized by changes in behavior in the initial stage of the disease, which may mask cognitive impairment ${ }^{5,37,38}$. An important reason for establishing an early diagnosis in YOD, besides those previously mentioned, is the relatively high occurrence of treatable causes of dementia in YOD. Comparative studies that compare the duration from symptom onset to diagnosis and the contribution of possible delaying factors between YOD and LOD have not been conducted. Furthermore, the subjective experiences of YOD caregivers regarding the period preceding diagnosis may provide clues to barriers and facilitators of receiving a timely diagnosis, which may have important implications for clinical practice.

\section{Neuropsychiatric symptoms}

Cognitive symptoms have long been seen as the hallmark symptoms of dementia, but the importance of non-cognitive symptoms is now widely recognized. Pearlin et al. (1990) already included problematic behavior as a primary objective stressor in their caregiver stress model, but it was only when the International Psychogeriatric Association in 1996 proposed the umbrella term psychological and behavioral symptoms in dementia (BPSD) that it became a widely acknowledged clinical problem. These symptoms, also called neuropsychiatric symptoms, are extremely common in dementia and have a negative impact on patients and caregivers.

The causes of neuropsychiatric symptoms are multifactorial and include biological, psychological and social factors. Therefore, differences in behavioral profiles between YOD and LOD are likely. In addition to the differences in etiologies, 
specific psychosocial factors related to younger age have been linked to a higher prevalence of behavioral problems in YOD, notably affective symptoms ${ }^{39}, 40$. The ecological model proposed by Lawton stresses the importance of balance between environmental demands and the capacity of an individual. According to this model, negative behavioral and affective outcomes will result when an individual's competence is not appropriate to deal with a given amount of pressure ${ }^{41}$. In individuals with YOD, the imbalance between their capacities and environmental demands may be higher than in the elderly, resulting in more behavioral symptoms. However, the few studies comparing neuropsychiatric symptoms between YOD and LOD show inconsistent results ${ }^{42-46}$, which could be due to heterogeneity in the methods used.

It is also possible that factors such as disease severity or level of awareness are involved. Awareness is an interesting phenomenon in this respect because previous research on LOD has suggested a positive association between awareness and affective symptoms ${ }^{47,48}$ and a negative association between awareness and age $^{48-50}$. In addition, many authors reported on the higher awareness of YOD patients $^{22,23,51}$, but to our knowledge, this has been based primarily on nonempirical studies.

To assess whether individuals with YOD are indeed a separate group in need of a specific approach in dementia care, the clinical characteristics should be investigated and compared with LOD. The presence of behavioral problems and level of awareness have specific management implications and are therefore important starting points. Behavioral problems are the most difficult to manage for caregivers $^{52}$ and are the most important predictor for patient admission into residential care ${ }^{52-54}$, while the level of awareness in turn has important associations with behavioral problems. To date, no large-scale longitudinal studies have been conducted in YOD, but these studies are necessary to obtain information regarding the risk factors, the course and the prognosis of behavioral symptoms. This knowledge is imperative for directing treatment, care and support for YOD patients and their caregivers, as this information provides clinicians the possibility to determine which patients are at risk and gives further insight into the underlying mechanisms of neuropsychiatric symptoms.

\subsection{Aims and outline of this thesis}

The central aim of this thesis is to assess the characteristics and impact of YOD for patients and their caregivers compared with LOD in light of their different psychosocial context. Three areas will be investigated: (1) the impact of dementia on caregivers, (2) the diagnostic process in dementia and (3) the development of neuropsychiatric symptoms during the course of dementia. Previous investigations concerning these topics have adopted qualitative and/or cross-sectional research designs and comparative studies comparing YOD with LOD are scarce. Therefore, we have conducted a 2-year prospective cohort study dubbed the Needs in Young- 
onset Dementia (NeedYD) study that obtained measurements every six months and included 215 YOD patients and their caregivers. Chapter 2 describes the participants, assessment methods and procedure of the NeedYD study in further detail. For most of the research questions, the results obtained in this study were compared with data from an historical cohort of LOD patients, the MAAstricht Study of BEhaviour in Dementia (MAASBED). In this thesis, we address the following research questions:

What is the psychosocial impact of YOD on informal caregivers including children and is this impact different from dementia at an older age?

Chapter 3 provides a review of the literature on the impact of YOD on informal caregivers including the patients' children. The purpose was to perform a systematic search and assessment of the available literature to characterize the impact of YOD on caregivers and gain insight into the differences from LOD.

How does the time to diagnosis differ between YOD and LOD, and what are the predictors of time to diagnosis?

Several studies have highlighted diagnostic difficulties as an important problem area in YOD, but there is a lack of studies comparing the time to diagnosis and delineating the influence of early age at onset and type of diagnosis. In chapter 4, we focused on the pre-diagnostic phase by comparing the time to diagnosis between YOD and LOD and examined the following predictors of time to diagnosis: age at onset (YOD vs. LOD), type of dementia, gender, education, living situation and family history of dementia.

What are the barriers to obtaining a diagnosis and what are the typical diagnosis pathways in $Y O D$ as experienced by caregivers?

Toward obtaining further insight into the pre-diagnostic phase, a qualitative study was conducted in chapter $\mathbf{5}$. The goal was to assess the possible barriers to diagnosis and to develop a typology of the pathway to diagnosis in YOD. None of the previously performed qualitative studies specifically focused on the prediagnostic period. A deeper exploration of the experiences of YOD caregivers during this period would provide issues to focus on in healthcare practice and future research.

What is the prevalence of neuropsychiatric symptoms in YO-AD compared to $L O-A D$ ? In chapter $\mathbf{6}$, the point- and cumulative prevalence, -incidence and -persistence of behavioral disturbances were compared between YO-AD and LOAD during the two-year period of this study. The proposition that affective symptoms are more common in YO-AD was investigated.

Is there a difference in the level of awareness and its association with affective symptoms between $Y O-A D$ and $L O-A D$ ? In the study presented in chapter 7, the level of awareness was compared between YO-AD and LO-AD as well as the 
relationship between awareness and affective symptoms. We hypothesized that awareness was higher in $\mathrm{YO}-\mathrm{AD}$ and that the association between awareness and affective symptoms was stronger in YO-AD than in LO-AD.

Finally, in chapter $\mathbf{8}$, the results of this thesis are summarized, the theoretical and methodological considerations discussed and implications for future research and directions for clinical practice are addressed. 


\section{References}

1. Goedert M. Oskar Fischer and the study of dementia. Brain 2009;132:1102-1111.

2. Maurer K, Volk S, Gerbaldo H. Auguste D and Alzheimer's disease. Lancet 1997;349:1546-1549.

3. Hodges JR. Alzheimer's centennial legacy: origins, landmarks and the current status of knowledge concerning cognitive aspects. Brain 2006;129:2811-2822.

4. Cipriani G, Dolciotti C, Picchi L, Bonuccelli U. Alzheimer and his disease: A brief history. Neurological Sciences 2011;32:275-279.

5. Mendez MF. The accurate diagnosis of early-onset dementia. International Journal of Psychiatry in Medicine 2006;36:401-412.

6. McMurtray A, McMurtray A, Clark DG, Christine D, Mendez MF. Early-Onset Dementia: Frequency and Causes Compared to Late-Onset Dementia. Dementia and Geriatric Cognitive Disorders 2006;21:59-64.

7. Armstrong M. The needs of people with young-onset dementia and their carers. Journal of Professional Nursing 2003;18:681-684.

8. Harris PB. The Perspective of Younger People with Dementia: Still an Overlooked Population. Social Work in Mental Health 2004;2:17-36.

9. Ferran J, Ferran J, Wilson K, Doran M, Ghadiali E. The early onset dementias: A study of clinical characteristics and service use. International Journal of Geriatric Psychiatry 1996;11:863-869.

10. Miyoshi K. What is early onset dementia? Psychogeriatrics 2009;9:67-72.

11. Rossor MN, Fox NC, Mummery CJ, Schott JM, Warren JD. The diagnosis of young-onset dementia. Lancet Neurology 2010;9:793-806.

12. Beattie A, Daker-White G, Gilliard J, Means R. 'How can they tell?' A qualitative study of the views of younger people about their dementia and dementia care services. Health \& Social Care in the Community 2004;12:359-368.

13. Wimo A, Jonsson L, Winblad B. An estimate of the worldwide prevalence and direct costs of dementia in 2003. Dementia and Geriatric Cognitive Disorders 2006;21:175-181.

14. Wimo A, Winblad B, Aguero-Torres H, von Strauss E. The magnitude of dementia occurrence in the world. Alzheimer Disease and Associated Disorders 2003;17:6367.

15. Harvey RJ, Skelton-Robinson M, Rossor MN. The prevalence and causes of dementia in people under the age of 65 years. Journal of Neurology, Neurosurgery $\&$ Psychiatry 2003;74:1206-1209.

16. Ikejima C, Yasuno F, Mizukami K, Sasaki M, Tanimukai S, Asada T. Prevalence and causes of early-onset dementia in Japan: a population-based study. Stroke 2009;40:2709-2714.

17. Mercy L, Hodges JR, Dawson K, Barker RA, Brayne C. Incidence of early-onset dementias in Cambridgeshire, United Kingdom. Neurology 2008;71:1496-1499.

18. Ratnavalli E, Brayne C, Dawson K, Hodges JR. The prevalence of frontotemporal dementia. Neurology 2002;58:1615-1621. 
19. Ridha B, Josephs KA. Young-onset dementia: a practical approach to diagnosis. The neurologist 2006;12:2-13.

20. Sampson EL, Warren JD, Rossor MN. Young onset dementia. Postgraduate Medical Journal 2004;80:125-139.

21. Bryan G, Martin CM. Unique problems of dementia in the younger patient. The Consultant Pharmacist 2005;20:468-479.

22. Reed J, Cantley C, Clarke CL, Stanley D. Services for younger people with dementia: Problems with differentiating needs on the basis of age. Dementia: The International Journal of Social Research and Practice 2002;1:95-112.

23. Tindall L, Manthorpe J. Early onset dementia: a case of ill-timing? Journal of Mental Health 1997;6:237-249.

24. Baumgarten M, Battista RN, Infante-Rivard C, Hanley JA, Becker R, Gauthier $\mathrm{S}$. The psychological and physical health of family members caring for an elderly person with dementia. Journal of Clinical Epidemiology 1992;45:61-70.

25. Schulz R, O'Brien AT, Bookwala J, Fleissner K. Psychiatric and physical morbidity effects of dementia caregiving: prevalence, correlates, and causes. The Gerontologist 1995;35:771-791.

26. Gallagher-Thompson D, Powers DV. Primary stressors and depressive symptoms in caregivers of dementia patients. Aging \& Mental Health 1997;1:248-255.

27. Beattie A, Daker-White G, Gilliard J, Means R. 'They don't quite fit the way we organize our services'--Results from a UK field study of marginalized groups and dementia care. Disability \& Society 2005;20:67-80.

28. Salloway S, Correia S. Alzheimer disease: time to improve its diagnosis and treatment. Cleveland Clinic Journal of Medicine 2009;76:49-58.

29. Piguet O, Halliday GM, Creasey H, Broe GA, Kril JJ. Frontotemporal dementia and dementia with Lewy bodies in a case-control study of Alzheimer's disease. International Psychogeriatrics 2009;21:688-695.

30. Dupiereux I, Zorzi W, Quadrio I, et al. Creutzfeldt-jakob, Parkinson, lewy body dementia and Alzheimer diseases: from diagnosis to therapy. Central Nervous System Agents in Medicinal Chemistry 2009;9:2-11.

31. Vernooij-Dassen MJFJ, Moniz-Cook ED, Woods RT, et al. Factors affecting timely recognition and diagnosis of dementia across Europe: From awareness to stigma. International Journal of Geriatric Psychiatry 2005;20:377-386.

32. Iliffe S, Robinson L, Brayne C, et al. Primary care and dementia: 1. diagnosis, screening and disclosure. International Journal of Geriatric Psychiatry 2009;24:895-901.

33. Wolfs CAG, Dirksen CD, Severens JL, Verhey FRJ. The added value of a multidisciplinary approach in diagnosing dementia: A review. International Journal of Geriatric Psychiatry 2006;21:223-232.

34. De Lepeleire J, Wind AW, Iliffe S, et al. The primary care diagnosis of dementia in Europe: An analysis using multidisciplinary, multinational expert groups. Aging Es Mental Health 2008;12:568-576. 
35. Sonnen JA, Montine KS, Quinn JF, Breitner JCS, Montine TJ. Cerebrospinal fluid biomarkers in mild cognitive impairment and dementia. Journal of Alzheimer's Disease 2010;19:301-309.

36. Milne A, Culverwell A, Guss R, Tuppen J, Whelton R. Screening for dementia in primary care: A review of the use, efficacy and quality of measures. International Psychogeriatrics 2008;20:911-926.

37. Jainer AK, Onalaja D, Noushad F. Anxiety can be a harbinger of early onset dementia. International Journal of Psychiatry in Clinical Practice 2005;9:299-300.

38. Kelley BJ, Boeve BF, Josephs KA. Cognitive and noncognitive neurological features of young-onset dementia. Dementia and Geriatric Cognitive Disorders 2009;27:564-571.

39. Rosness TA, Barca ML, Engedal K. Occurrence of depression and its correlates in early onset dementia patients. International Journal of Geriatric Psychiatry 2010;25:704-711.

40. Savva GM, Zaccai J, Matthews FE, Davidson JE, McKeith I, Brayne C. Prevalence, correlates and course of behavioural and psychological symptoms of dementia in the population. British Journal of Psychiatry 2009;194:212-219.

41. Izal M, Montorio I, Marquez M, Losada A. Caregivers' expectations and care receivers' competence Lawton's ecological model of adaptation and aging revisited. Archives of Gerontology and Geriatrics 2005;41:129-140.

42. Toyota Y, Ikeda M, Shinagawa S, et al. Comparison of behavioral and psychological symptoms in early-onset and late-onset Alzheimer's disease. International Journal of Geriatric Psychiatry 2007;22:896-901.

43. Papageorgiou SG, Kontaxis T, Bonakis A, Kalfakis N, Vassilopoulos D. Frequency and causes of early-onset dementia in a tertiary referral center in Athens.

Alzheimer Disease and Associated Disorders 2009;23:347-351.

44. Borroni B, Agosti C, Bellelli G, Padovani A. Is early-onset clinically different from late-onset frontotemporal dementia? European Journal of Neurology 2008;15:14121415 .

45. Shinagawa S, Toyota Y, Ishikawa $\mathrm{T}$, et al. Cognitive function and psychiatric symptoms in early- and late-onset frontotemporal dementia. Dementia and Geriatric Cognitive Disorders 2008;25:439-444.

46. Hori K, Oda T, Asaoka T, et al. First episodes of behavioral symptoms in Alzheimer's disease patients at age 90 and over, and early-onset Alzheimer's disease: comparison with senile dementia of Alzheimer's type. Psychiatry and Clinical Neurosciences 2005;59:730-735.

47. Aalten P, Van Valen E, Clare L, Kenny G, Verhey F. Awareness in dementia: A review of clinical correlates. Aging \& Mental Health 2005;9:414-422.

48. Aalten P, van Valen E, de Vugt ME, Lousberg R, Jolles J, Verhey FRJ. Awareness and behavioral problems in dementia patients: A prospective study. International Psychogeriatrics 2006;18:3-17.

49. Verhey FR, Rozendaal N, Ponds RW, Jolles J. Dementia, awareness and depression. International Journal of Geriatric Psychiatry 1993;8:851-856. 
50. Derouesne C, Thibault S, Lagha-Pierucci S, Baudouin-Madec V, Ancri D, Lacomblez L. Decreased awareness of cognitive deficits in patients with mild dementia of the Alzheimer type. International Journal of Geriatric Psychiatry 1999;14:10191030 .

51. Beattie AM, Daker-White G, Gilliard J, Means R. Younger people in dementia care: a review of service needs, service provision and models of good practice. Aging $\mathcal{G}$ Mental Health 2002;6:205-212.

52. Ballard C, Lowery K, Powell I, O'Brien J, James I. Impact of behavioral and psychological symptoms of dementia on caregivers. International Psychogeriatrics 2000;12:93-105.

53. Donaldson C, Tarrier N, Burns A. The impact of the symptoms of dementia on caregivers. British Journal of Psychiatry 1997;170:62-68.

54. Mausbach BT, Patterson TL, Rabinowitz YG, Grant I, Schulz R. Depression and distress predict time to cardiovascular disease in dementia caregivers. Health Psychology 2007;26:539-544. 


\title{
Chapter 2
}

\section{Research protocol of the NeedYD-study}

\author{
a prospective cohort study on the needs \\ and course of young-onset dementia
}

Based on: van Vliet D, Bakker C, Koopmans RTCM, Vernooij-Dassen MJFJ, Verhey FRJ, de Vugt ME. (2010). Research protocol of the NeedYD-study (Needs in Young onset Dementia): a prospective cohort study on the needs and course of young-onset dementia. BMC geriatrics, 10, 13. 


\begin{abstract}
Background Young-onset dementia has serious consequences for patients and their family members. Although there has been growing attention for this patient group, health care services are still mainly targeted at the elderly. Specific knowledge of the needs of young-onset dementia patients and their families is limited but necessary for the development of adequate health care services and specific guidelines. This research project is mainly targeted at delineating the course of young-onset dementia, the functional characteristics and needs of young- onset dementia patients and their caregivers, the risk factors for institutionalization and the interaction with the caring environment.

Methods The NeedYD-study (Needs in Young-onset Dementia) is a longitudinal observational study investigating young-onset dementia patients and their caregivers $(\mathrm{n}=215)$. Assessments were performed every six months over two years and consisted of interviews and questionnaires with patients and caregivers. The main outcomes were (1) the needs of patients and caregivers, as measured by the Camberwell Assessment of Needs for the Elderly (CANE) and (2) neuropsychiatric symptoms, as measured by the NeuroPsychiatric Inventory (NPI). Qualitative analyses were performed in order to obtain more in-depth information on the experiences of young-onset dementia patients and their family members. The results of this study were compared with comparable data on late-onset dementia from a historical cohort.
\end{abstract}

Discussion The study protocol of the NeedYD-study is presented here. To our knowledge, this study is the first prospective cohort study in this research area. Although some limitations exist, these do not outweigh the strengths of this study design. 


\subsection{Background}

Dementia is often regarded as a disease of old age. However, there is also a group in which the symptoms of the disease develop before the age of 65 . Prevalence rates of young-onset dementia (YOD) have been reported to range between 54 and 260 cases per 100,000 in the $30-64$ age group ${ }^{1-3}$.

YOD is recognized as an important psychosocial and medical health problem with serious consequences for patients and their families ${ }^{4,5}$. YOD is more difficult to recognize than late- onset dementia (LOD) in the early stages of the disease because of the lower prevalence rate, the wider range of etiologies ${ }^{6,7}$ and the use of other mental health services (e.g., community mental health teams). These factors cause an important delay before an accurate diagnosis can be established, commonly resulting in feelings of insecurity and frustration for both patients and their families ${ }^{8}$. A proper diagnosis is an important prerequisite for receiving adequate (in)formal support and health care services.

YOD also may have a different clinical manifestation than LOD due to the relatively high prevalence rate of frontotemporal dementia (FTD), in which problem behavior is more prevalent as the presenting sign of probable dementia ${ }^{6,7}$. Recent research on the impact of problem behavior on caregivers and vice versa shows that these specific aspects of the dementia, more so than cognitive and functional changes, have severe consequences for patients and their family members 9. Problem behavior is the most important risk factor for caregiver burden and is a strong predictor of institutionalization ${ }^{10-12}$ but is also an important starting point for interventions ${ }^{13}$. Recent studies have shown that psychological factors, such as disease awareness ${ }^{14}$, and environmental factors, such as caregiver management strategies ${ }^{15}$, influence the development and persistence of problem behavior in LOD. Similar studies on YOD have not yet been conducted.

Furthermore, YOD patients are in a life phase in which they often have an active role in society and often have young children. The loss of roles and responsibilities is, therefore, greater than in older people. They also have to deal with specific issues such as marital problems, family conflict, (un)employment and financial issues ${ }^{5}$. Furthermore, many YOD patients of the post-war baby boom generation grew up in a society that is very different from that of the older generation. The needs of YOD patients may, therefore, be different and demand a different approach than in LOD.

Despite these differences, the availability of specialized healthcare services is still limited in most countries, forcing YOD patients and their family members to use services designed for the elderly. In the Netherlands, specialized services are available, but their geographic distribution is limited, as is the range of services offered. Furthermore, specific knowledge on the characteristics and needs of YOD patients and their families is lacking but prerequisite for the development of suitable health care services. Adequate diagnostics, (in)formal support and services like support groups, day care facilities or respite care may help patients and their families cope with the situation and may even postpone institutionalization. This 
multidisciplinary research project focuses on the course of YOD, the functional characteristics of YOD patients, the needs of YOD patients and their caregivers, the risk factors for institutionalization and the interaction with the caring environment. We expect that the study will yield important data that can be used to design specific guidelines and improve the development of health care services for YOD patients and their families.

\section{Aim and research questions}

NeedYD is a prospective cohort study with the following primary objectives: (1) to investigate the (un)met needs of YOD patients and their family members during different phases in the course of the disease (e.g., the diagnostic phase and the phase in which (specialized) day care is provided) and (2) to investigate the course of neuropsychiatric symptoms and possible risk factors (e.g., comorbidity, age, communication problems, biological factors, disease awareness, interaction with environment). The secondary objectives are:

- To gain insight into the course of other functional domains in YOD (cognition, activities of daily living);

- To explore the experiences and feelings of patients and their caregivers during the diagnostic period;

- To investigate the impact of the diagnosis of dementia on YOD patients and their family members;

- To study the course of functioning of the caregivers of YOD patients and the problems they (and possibly other family members) experience;

- To identify factors that influence the use of respite care and determine institutionalization;

- To explore to what extent stigma and taboo concerning dementia interfere with adequate communication within the family;

- To compare these findings with findings of studies on LOD.

\subsection{Methods}

\section{Design}

The NeedYD-study is a prospective cohort study with a follow-up of two years in which a group of YOD patients and their families were assessed at six month intervals. The study design is similar to that of the MAAstricht Study of BEhavior in Dementia (MAASBED) and the WAAL BEhavior in Dementia (WAALBED) study ${ }^{16-19}$ conducted in the Netherlands. 


\section{Subjects}

The study population consisted of dyads of patients with YOD and their caregivers. Patients with onset of disease symptoms before the age of 65 were included in the study (age at inclusion could be later than 65). Diagnoses of dementia subtype were made according to regular criteria ${ }^{20-25}$. Patients were recruited through the memory clinics of the three Alzheimer's centers in the Netherlands located in Amsterdam, Nijmegen and Maastricht, the memory clinics of general hospitals and through other mental health services in the south of the Netherlands as well as through specialized day care facilities that are affiliated with the Dutch National YOD Taskforce. Thus, a group of patients without day care or receiving nonspecialized day care, as well as a group of patients receiving specialized day care, were included in the study. For some of the research questions, these groups will be compared. The exclusion criteria were: (1) dementia caused by HIV, traumatic brain injury, Down's syndrome, Huntington's chorea or alcohol-related dementia, (2) lack of a reliable informant or (3) lack of informed consent of the participant. Furthermore, children of YOD patients who were living at home and were older than 14 years of age at the time of the baseline assessments were recruited through their parents.

\section{Measures}

Primary outcome measures (Un)met needs were assessed with the Dutch version of the Camberwell Assessment of Needs in the Elderly (CANE) ${ }^{26}$. This assessment is a semi-structured interview consisting of 24 domains that cover social, physical, psychological and environmental needs. The interview started with an open question concerning a specific domain, followed by questions regarding help and (in)formal support the patient receives in that particular domain, as well as the amount of help and support that was needed. These items were scored on a three point scale ranging from little (1) to a lot of help (3). Satisfaction with the amount and quality of the help and support received was also assessed. The answers were used to determine whether or not the participant experienced a need and whether or not this need was met. The experienced needs of patients were based on patient and proxy (primary caregiver) interviews. The need for information and the psychological needs of the caregiver were also assessed by means of the CANE. Reliability and validity were found to be adequate ${ }^{26}$.

Neuropsychiatric symptoms in the patient and related caregiver burden were assessed with the Dutch version of the Neuropsychiatric Inventory (NPI) ${ }^{27}$. The NPI is a structured interview with the primary caregiver and, when available, a health care professional. After institutionalization, the nursing home version of the NPI (NPI-NH) was used ${ }^{28}$. 


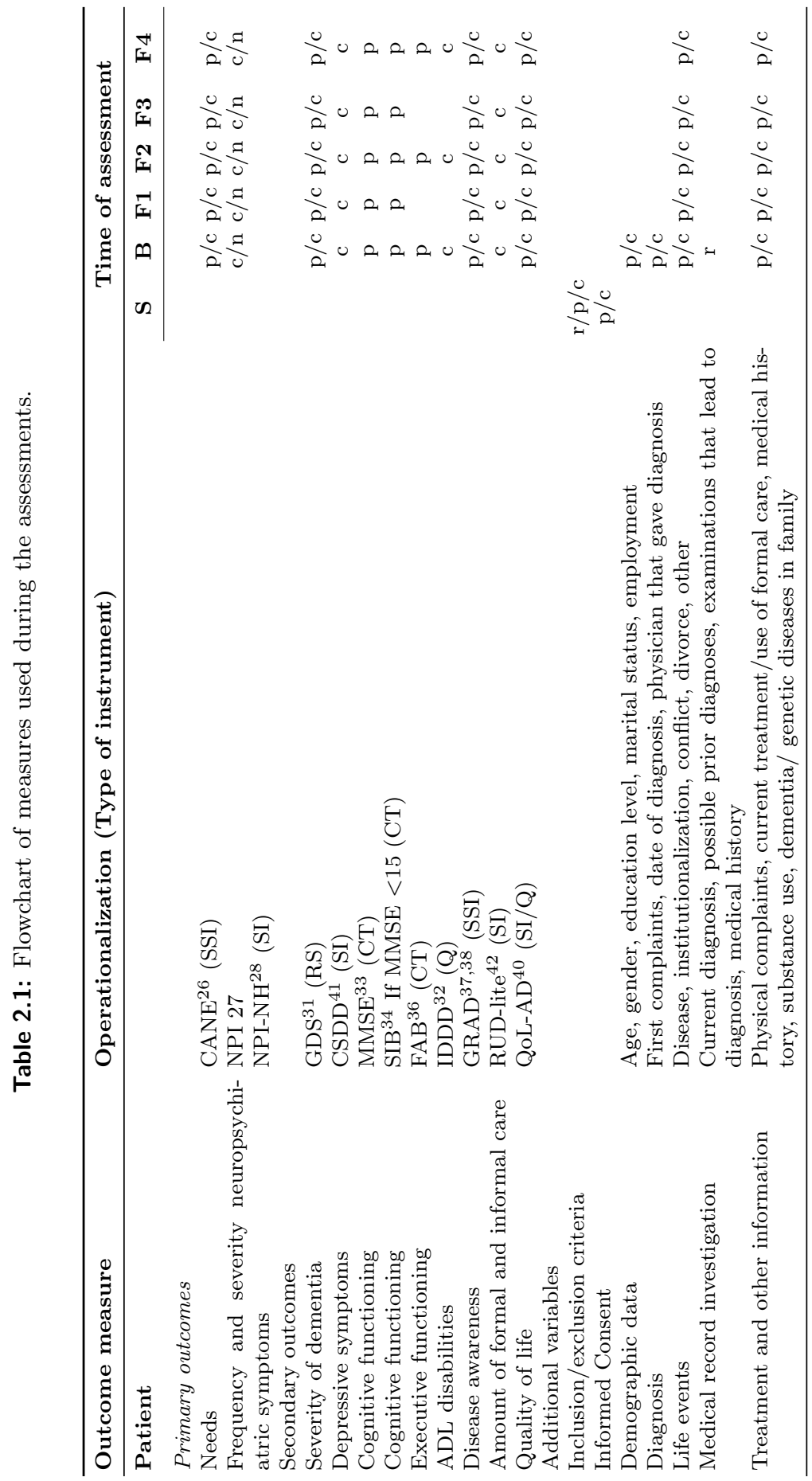




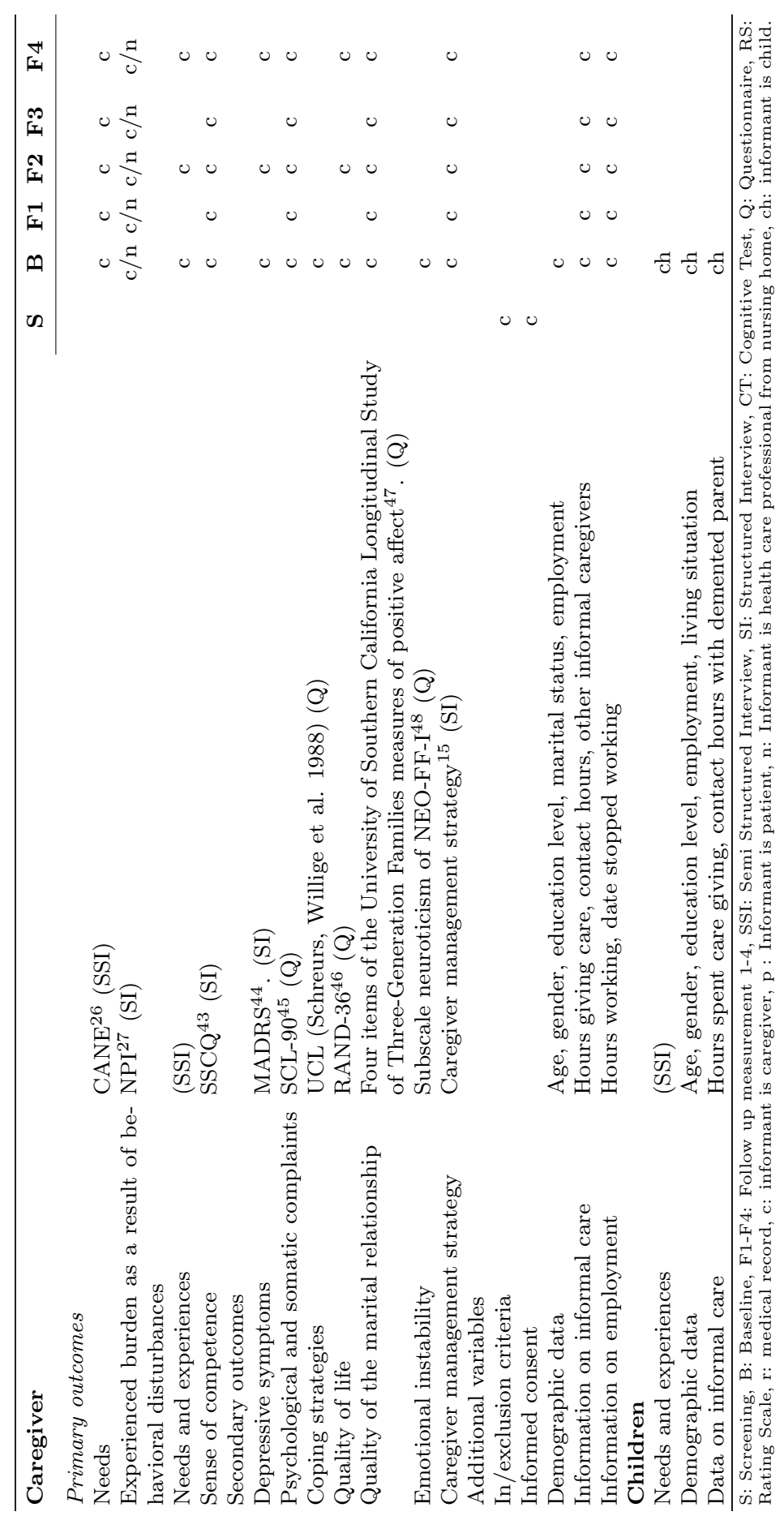


Ten neuropsychiatric and two neuro-vegetative symptoms were assessed: delusions, hallucinations, agitation/aggression, dysphoria, anxiety, euphoria, apathy, disinhibition, irritability/lability, aberrant motor behavior, night-time behavior disturbances and appetite/eating abnormalities. Screening questions were asked to determine whether behavioral changes were present. In the case of a positive answer, further questions were asked and the severity and frequency of the behavioral disturbances were determined. The Dutch version of the NPI was found to have high inter-rater agreement and to be a valid rating scale for measuring a wide range of behavioral and psychological symptoms of dementia ${ }^{29}$. Furthermore, the experience of caregiver distress due to these neuropsychiatric symptoms was determined according to the six point NPI caregiver distress scale (NPI-D) ranging from no distress $(0)$ to extreme distress $(5)^{30}$. The NPI-D provides a reliable and valid measure of subjective caregiver distress in relation to the neuropsychiatric symptoms measured by the NPI.

For an overview of all measurements see Table 2.1.

Secondary outcome measures for the patient The Global Deterioration Scale (GDS) was administered to assess the severity of the dementia. The GDS is a widely used instrument which has been validated against behavioral, neuroanatomic and neurophysiologic measures, for which significant correlations have been found ${ }^{31}$. The Interview for Deterioration in Daily Living in Dementia (IDDD) was used to assess the activities of daily living. The internal consistency of this scale is high (Cronbach's alpha 0.94) ${ }^{32}$. Cognitive functioning was measured using the Mini Mental State Examination (MMSE), which is a reliable and valid test of cognitive function ${ }^{33}$. When the MMSE score was below 15, the Short Severe Impairment Battery (s-SIB) was administered, which has been found to be a reliable and valid test of cognitive function in moderate to severe dementia patients $^{34,35}$. Furthermore, executive functioning was assessed using the Frontal Assessment Battery (FAB). The FAB has good inter-rater reliability, internal consistency and discriminant validity ${ }^{36}$. The Guidelines for the Rating of Awareness of Deficits (GRAD) ${ }^{37,} 38$ were administered in order to investigate disease awareness. This instrument has substantial inter-rater reliability ${ }^{39}$. The Quality of Life-Alzheimer's Disease scale (QoL-AD), which has good content, criterion and construct validity and excellent inter-rater reliability and internal consistency ${ }^{40}$, was used to assess the quality of life of the patient, as perceived by the patient and his caregiver. The Cornell Scale for Depression in Dementia (CSDD) ${ }^{41}$ was administered to identify depressive symptoms in the patient. This scale has adequate inter-rater reliability, internal consistency and sensitivity. The amount of formal care the patient receives and the time the caregiver spends caring for the patient were obtained using the Resource Utilization Scale (RUD-Lite), which covers $95 \%$ of the resource use, the complete RUD covers ${ }^{42}$. Therefore, it is a good alternative for the complete RUD when the assessment battery is large. 
Secondary outcome measures for the caregiver The Short Sense of Competence Questionnaire (SSCQ) ${ }^{43}$ was administered to assess caregiver's feelings of being capable to care for a demented individual. The SSCQ was reported to have satisfactory reliability and validity ${ }^{43}$. Depressive symptoms were measured by the Montgomery Asberg Depression Rating Scale (MADRS), which has adequate inter-rater reliability and exhibits construct and concurrent validity ${ }^{44}$. Psychological and physical complaints were measured with the Symptom Checklist 90 (SCL90). Reliability and construct validity of the SCL-90 are satisfactory ${ }^{45}$. Emotional instability was assessed with the neuroticism subscale of the Dutch version of the NEO- Five Factor Inventory (NEO-FF-I). Internal consistency and test-retest reliability are high for this scale, as is the construct validity ${ }^{49}$. Coping strategies were assessed by means of the Utrechtse Copinglijst (UCL). The reliability of this scale is reasonable and the validity has been found to be sufficient despite some inconsistencies in the literature ${ }^{50}$. General health was measured with the Dutch translation of the RAND- $36^{46}$. The Dutch version of the RAND-36 appears to be a reliable, valid and sensitive measure for general health ${ }^{51}$. In addition, the quality of the marital relationship and the changes that have occurred since the onset of the disease were measured by four items of the University of Southern California Longitudinal Study of Three-Generation Families measures of positive affect. Cronbach's alpha for this scale is $0.85^{47}$. The caregiver management strategy was assessed by means of questions reflecting three caregiver strategies: a caring, supporting or non-adapting strategy ${ }^{15}$. This scale has not yet been validated. Furthermore, a semi-structured interview was administered to the caregiver and, when applicable, to children living at home. The interview included topics concerning the period prior to diagnosis, the impact of the diagnosis, changes in the interpersonal relationships within the family, the communication within the family about the disease, the problems experienced by the patient and family members, experiences and beliefs concerning (in)formal support and health care services, transitions in care (e.g., day care, institutionalization) and future perspectives.

Additional data By means of a structured interview and examining the patients file information, medical and demographical information of the patient were obtained. For a full description of these data, see 2.1.

\section{Procedures}

YOD patients and their caregivers received five assessments at six-month intervals (B, F1, F2, F3, F4; 2.1). Before inclusion in the study, (S) information on in/exclusion criteria was collected and informed consent was obtained. Patients who were not able to sign informed consent were asked to give oral consent and their legal representative had to give written consent that the patient was able to participate. Children living at home who were older than age 14 were asked at baseline to participate in a semi-structured interview. Children aged between 15 and 18 years, as well as their legal representatives, both had to sign informed con- 
sent. When individuals did not agree to participate, the reason plus age, gender and diagnosis of the patients were registered.

When participants, after inclusion in the study, did not wish to participate in one of the assessments, caregivers were asked to participate in an interview by telephone, so the CANE, NPI and Sense of Competence questionnaire could still be administered and to fill out all of the questionnaires required for that assessment. If this was not possible, the researcher asked them to answer several questions about their own and the patients' functioning and about the use of formal care. If caregivers refused this as well, the reason for refusal was asked. When a patient had died, data on the use of (in)formal care and the needs of caregivers before and after the patient died was collected from the caregiver as well as the date and cause of death.

\section{Ethical considerations}

The study protocol was approved by the Medical Ethics Committee of the University Medical Center Maastricht. The local ethics committees of the participating institutions have also given consent. The research project was performed according to the principles of the Declaration of Helsinki (version January 2004; http://www.wma.net) and in agreement with the law regarding medical-scientific research in humans (WMO). An independent physician was assigned to the study. Participants were informed about the possibility of contacting him for further questions about the study.

\section{Sample size}

Based on a power calculation (two groups: diagnostic phase and the phase of specialized day care; ANOVA) with an alpha of 0.05 , a power of 0.85 and an expected effect size of $0.25,128$ YOD patients were required to participate in the study. With an expected loss to follow-up of $37 \%$ in a two year follow-up period based on data of the MAAstricht Study of BEhavior in Dementia (MAASBED) study ${ }^{15}, 200$ patients needed to be included.

\section{Data analysis}

Data entry was performed twice to safeguard data integrity. Statistical analyses were performed using the Statistical Package for Social Sciences. Descriptive statistics were used to describe characteristics of patients and caregivers, i.e., age, sex, distribution of diagnoses, etc. Both quantitative and qualitative data were used in the analyses.

Diagnosis matched patients with LOD from a historical cohort (MAASBED study) were used to make a comparison with YOD for some of the research questions. Baseline differences between groups were analyzed to investigate the comparability of the groups. Depending on the research question and which variables 
were analyzed, parametric or non-parametric analyses were performed. Comparisons between groups were made with independent samples t-tests or AN(C)OVAs for continuous and normally distributed variables and Pearson's Chi square test, Fisher's exact or Mann-Whitney U tests for categorical and non-normally distributed variables. Comparisons between the memory clinic and day care groups, the YOD group and the LOD group and within groups across measurements will be performed using linear mixed models analyses. A survival analysis will be performed to study predictors of institutionalization. If participants withdrew from the study, they were not excluded. The data collected could still be analyzed, because of the use of linear mixed models. However, the characteristics of the dropouts and losses to follow-up were described and taken into account.

Qualitative data were analyzed using the method of constant comparative analysis $^{52}$. These qualitative analyses were performed in order to obtain a more in-depth understanding of the experiences of YOD patients and their family members. The interviews that were held with the caregivers were transcribed and first read by one researcher. They were then read a second time to develop codes that were grouped into categories. Categories were grouped into themes. Another researcher independently applied the same procedure. The analyses were performed using Atlas.ti.

\subsection{Discussion}

The current paper presents the study protocol of a prospective cohort study: the NeedYD-study. This project mainly focuses on the course of YOD, the functional characteristics of YOD patients, the needs of YOD patients and their caregivers, the risk factors for institutionalization and the interaction with the caring environment. To our knowledge, this is the first study that addresses these issues longitudinally in a large cohort. It will contribute widely to our knowledge about the course of YOD, the caring process and the needs of the patient and caregiver as they develop during the course of the disease. A longitudinal design is necessary to examine the predictive value of study variables in observational data. Despite the many positive aspects of this design, there are some limitations.

Sample bias could be a factor in our study. Although patients are recruited through a large range of different institutions, which is likely to be representative of the Dutch population, the group that gives consent may be different from the group that refuses to participate. Furthermore, selective attrition due to early death is inherently associated with the current study. In addition, the data from the present study and the historical cohort of the LOD sample (MaasBED study) are different in several ways, as the participants are not matched. The LOD patients were mostly seen right after receiving the diagnosis, whereas in the YOD group patients in different stages are included. The YOD group is, therefore, probably more heterogeneous in terms of disease severity, cognitive functioning and ADL disability. Furthermore, the dementia of the YOD patients is possibly 
more severe because establishing a diagnosis in YOD often takes longer than in LOD. However, these factors as well as other possible confounders are collected in order to take these into account during the statistical analyses. Furthermore, one may argue that the proxy ratings we use for several patient characteristics are not as reliable as patient ratings. However, in this patient group such ratings are inevitable since dementia patients gradually become cognitively impaired and may suffer from a lack of awareness. Therefore, proxy ratings are preferred to keep the informant during follow-up reliable and constant.

In conclusion, the strengths of this study outweigh its few limitations as long as they are dealt with properly. 


\section{References}

1. Harvey RJ, Rossor MN, Skelton-Robinson M, Garralda E. Young onset dementia: epidemiology, clinical symptoms, family burden, support and outcome. Imperial college of science, technology and medicine Dementia research group 1998.

2. Harvey RJ, Skelton-Robinson M, Rossor MN. The prevalence and causes of dementia in people under the age of 65 years. Journal of Neurology, Neurosurgery $\&$ Psychiatry 2003;74:1206-1209.

3. Sulkava R. Prevalence of severe dementia in Finland. Neurology 1985;35:1025-1029.

4. Werner P, Stein-Shvachman I, Korczyn AD. Early onset dementia: clinical and social aspects. International Psychogeriatrics 2009:1-6.

5. van Vliet D, de Vugt ME, Bakker C, Koopmans R, Verhey FRJ. Impact of early onset dementia on caregivers: a review. International Journal of Geriatric Psychiatry.

6. Sampson EL, Warren JD, Rossor MN. Young onset dementia. Postgraduate Medical Journal 2004;80:125-139.

7. Mendez MF. The accurate diagnosis of early-onset dementia. International Journal of Psychiatry in Medicine 2006;36:401-412.

8. van Vliet D, Bakker C, de Vugt ME, Koopmans RTCM, Verhey FRJ. How do caregivers of patients with early onset dementia perceive the period prior to diagnosis. In: Abstract book of the 19th Alzheimer Europe Conference: 28-30 May, 2009, Brussels, Errata Corrige. 2009: 2.

9. de Vugt ME, Stevens F, Aalten P, et al. Behavioural disturbances in dementia patients and quality of the marital relationship. International Journal of Geriatric Psychiatry 2003;18:149-154.

10. Knopman DS, Kitto J, Deinard S, Heiring J. Longitudinal study of death and institutionalization in patients with primary degenerative dementia. Journal of the American Geriatrics Society 1988;36:108-112.

11. Steele C, Rovner B, Chase GA, Folstein M. Psychiatric symptoms and nursing home placement of patients with Alzheimer's disease. American Journal of Psychiatry 1990;147:1049-1051.

12. Gaugler JE, Yu F, Krichbaum K, Wyman JF. Predictors of nursing home admission for persons with dementia. Medical Care 2009;47:191-198.

13. de Vugt ME, Stevens F, Aalten P, Lousberg R, Jaspers N, Verhey FRJ. A prospective study of the effects of behavioral symptoms on the institutionalization of patients with dementia. International Psychogeriatrics 2005;17:577-589.

14. Aalten P, van Valen E, de Vugt ME, Lousberg R, Jolles J, Verhey FRJ. Awareness and behavioral problems in dementia patients: A prospective study. International Psychogeriatrics 2006;18:3-17.

15. de Vugt ME, Stevens F, Aalten P, et al. Do caregiver management strategies influence patient behaviour in dementia? International Journal of Geriatric Psychiatry 2004;19:85-92. 
16. Zuidema SU, de Jonghe JFM, Verhey FRJ, Koopmans RTCM. Neuropsychiatric symptoms in nursing home patients: Factor structure invariance of the Dutch nursing home version of the Neuropsychiatric Inventory in different stages of dementia. Dementia and Geriatric Cognitive Disorders 2007;24:169-176.

17. Zuidema SU, Derksen E, Verhey FRJ, Koopmans RTCM. Prevalence of neuropsychiatric symptoms in a large sample of Dutch nursing home patients with dementia. International Journal of Geriatric Psychiatry 2007;22:632-638.

18. Aalten P, de Vugt ME, Jaspers N, Jolles J, Verhey FRJ. The course of neuropsychiatric symptoms in dementia. Part I: Findings from the two-year longitudinal Maasbed study. International Journal of Geriatric Psychiatry 2005;20:523-530.

19. de Vugt ME, Riedijk SR, Aalten P, Tibben A, van Swieten JC, Verhey FR. Impact of behavioural problems on spousal caregivers: a comparison between Alzheimer's disease and frontotemporal dementia. Dementia and Geriatric Cognitive Disorders 2006;22:35-41.

20. American Psychiatric Association. Diagnostic and Statistical manual of mental Disorders- Text revision: DSM-IV-TR. American Psychiatric Association 2000.

21. McKhann G, Drachman D, Folstein M, Katzman R, Price D, Stadlan EM. Clinical diagnosis of Alzheimer's disease: report of the NINCDS-ADRDA Work Group under the auspices of Department of Health and Human Services Task Force on Alzheimer's Disease. Neurology 1984;34:939-944.

22. McKeith IG. Consensus guidelines for the clinical and pathologic diagnosis of dementia with Lewy bodies (DLB): report of the Consortium on DLB International Workshop. Journal of Alzheimer's Disease 2006;9:417-423.

23. Neary D, Snowden JS, Gustafson L, et al. Frontotemporal lobar degeneration: a consensus on clinical diagnostic criteria. Neurology 1998;51:1546-1554.

24. Mesulam MM, Grossman M, Hillis A, Kertesz A, Weintraub S. The core and halo of primary progressive aphasia and semantic dementia. Annals of Neurology 2003;54 Suppl 5:S11-14.

25. Erkinjuntti T. Clinical criteria for vascular dementia: The NINDS-AIREN criteria. Dementia 1994;5:189-192.

26. Reynolds T, Thronicroft G, Abas M, et al. Camberwell Assessment of Need for the Elderly (CANE): Development, validity and reliability. British Journal of Psychiatry 2000;176:444-452.

27. Cummings JL, Mega M, Gray K, Rosenberg-Thompson S. The Neuropsychiatric Inventory: Comprehensive assessment of psychopathology in dementia. Neurology 1994;44:2308-2314.

28. Wood S, Cummings JL, Hsu M-A, et al. The use of the Neuropsychiatric Inventory in nursing home residents: Characterization and measurement. American Journal of Geriatric Psychiatry 2000;8:75-83.

29. Kat MG, de Jonghe JFM, Aalten P, Kalisvaart CJ, Dres RM, Verhey FRJ. Neuropsychiatrische symptomen bij dementie: Psychometrische aspecten van de Nerlandse Neuropsychiatric Inventory (NPI). Tijdschrift voor Gerontologie en Geriatrie 2002;33:150-155. 
30. Kaufer DI, Cummings JL, Christine D, et al. Assessing the impact of neuropsychiatric symptoms in Alzheimer's disease: The Neuropsychiatric Inventory Caregiver Distress Scale. Journal of the American Geriatrics Society 1998;46:210-215.

31. Reisberg B, Ferris SH, de Leon MJ, Crook T. The Global Deterioration Scale for assessment of primary degenerative dementia. American Journal of Psychiatry 1982;139:1136-1139.

32. Teunisse S, Derix MM. Meten van het dagelijks functioneren van thuiswonende dementiepatinten: ontwikkeling van een vragenlijst. Tijdschrift voor Gerontologie en Geriatrie 1991;22:53-59.

33. Folstein MF, Folstein SE, McHugh PR. Mini-mental state: A practical method for grading the cognitive state of patients for the clinician. Journal of Psychiatric Research 1975;12:189-198.

34. Saxton J, Kastango KB, Hugonot-Diener L, et al. Development of a Short Form of the Severe Impairment Battery. American Journal of Geriatric Psychiatry 2005;13:999-1005.

35. de Jonghe JF, Wetzels RB, Mulders A, Zuidema SU, Koopmans RT. Validity of the Severe Impairment Battery Short Version. Journal of Neurology, Neurosurgery, and Psychiatry 2009;80:954-959.

36. Dubois B, Slachevsky A, Litvan I, Pillon B. The FAB: A frontal assessment battery at bedside. Neurology 2000;55:1621-1626.

37. Verhey FR, Rozendaal N, Ponds RW, Jolles J. Dementia, awareness and depression. International Journal of Geriatric Psychiatry 1993;8:851-856.

38. Zanetti O, Vallotti B, Frisoni GB, et al. Insight in dementia: When does it occur? Evidence for a nonlinear relationship between insight and cognitive status. Journals of Gerontology: Series B: Psychological Sciences and Social Sciences 1999:P100-p106.

39. Verhey FRJ, Ponds RWHM, Rozendaal N, Jolles J. Depression, insight, and personality changes in Alzheimer's disease and vascular dementia. Journal of Geriatric Psychiatry and Neurology 1995;8:23-27.

40. Thorgrimsen L, Selwood A, Spector A, et al. Whose Quality of Life Is It Anyway? The Validity and Reliability of the Quality of Life-Alzheimer's Disease (QoL-AD) Scale. Alzheimer Disease \& Associated Disorders 2003;17:201-208.

41. Alexopoulos GS, Abrams RC, Young RC, Shamoian CA. Cornell Scale for Depression in Dementia. Biological Psychiatry 1988;23:271-284.

42. Wimo A, Winblad B. Resource Utilization in Dementia: "RUD Lite". Brain Aging 2003;3:48-59.

43. Vernooij-Dassen MJ, Felling AJ, Brummelkamp E, Dauzenberg MG, van den Bos GA, Grol R. Assessment of caregiver's competence in dealing with the burden of caregiving for a dementia patient: a Short Sense of Competence Questionnaire (SSCQ) suitable for clinical practice. Journal of the American Geriatrics Society 1999;47:256-257.

44. Davidson J, Turnbull CD, Strickland R, Miller R. The Montgomery-Asberg Depression Scale: Reliability and validity. Acta Psychiatrica Scandinavica 1986;73:544548. 
45. Arindell WA, Ettema JHM. SCL-90 Herziene handleiding bij een multidimensionele psychopathologie indicator. Lisse: Swets Publishers, 2003.

46. Hays RD, Morales LS. The RAND-36 measure of health-related quality of life. Annals of Medicine 2001;33:350-357.

47. Lawrence ReH, Tennstedt SL, Assmann SF. Quality of the caregiver-care recipient relationship: Does it offset negative consequences of caregiving for family caregivers? Psychology and Aging 1998;13:150-158.

48. Costa PT, McCrae RR. Normal personality assessment in clinical practice: The NEO Personality Inventory. Psychological Assessment 1992;4:5-13.

49. Costa PTJ, McCrae RR. Revised NEO Personality Inventory (NEO-PI-R) and NEO Five-Factor Inventory (NEO-FFI) Professional Manual. Odessa FL: Psychological Assessment Resources, 1992b.

50. Schreurs PJG, Willige G, Brosschot JF, Telegen B, Graus GMH. Handleiding Utrechtse Coping Lijst UCL (herziene versie). Lisse: Swets \& Zeitlinger, 1993.

51. Van der Zee KI, Sandemann R. Het meten van de algemene gezondheidstoestand met de RAND-36: een handleiding (Measurement of general health with the RAND-36: a manual).

52. Corbin J, Strauss A. Grounded Theory Research: Procedures, Canons, and Evaluative Criteria: Springer Science \& Business Media B.V., 1990. 


\section{Chapter 3}

\section{Impact of young-onset dementia on caregivers: a review}

Based on: van Vliet D, de Vugt ME, Bakker C, Koopmans RTCM. and Verhey FRJ (2010). Impact of early onset dementia on caregivers: a review. International Journal of Geriatric Psychiatry, 25, 1091-100. 


\begin{abstract}
Objective When it comes to dementia, caregiving can have adverse effects on the psychological and physical health of the informal caregiver. As yet, little is known about the impact of caring for a young dementia patient. This review provides an overview of the literature concerning the impact of young-onset dementia (YOD) on informal caregivers and on children of YOD patients. The available literature comparing the impact on YOD and late-onset dementia (LOD) caregivers will also be provided.

Methods PubMed, Psychinfo and Cinahl were searched for articles that considered the psychological or psychosocial impact of YOD on informal caregivers and children. The methodological quality of the studies was assessed in order to make better judgments about the value of each article.

Results Seventeen articles were included, of which the overall methodological quality was limited. The results showed that YOD caregivers experienced high levels of burden, stress and depression. When compared with LOD caregivers, results were inconclusive. Furthermore, the caregivers of YOD patients experienced a variety of psychosocial problems, including relational problems, family conflict, problems with employment, financial difficulties and problems concerning diagnosis.

Conclusions Whether there is a difference in impact between YOD and LOD on caregivers is still unclear. The studies conducted are methodologically too limited to answer this question. Nevertheless, it is clear that YOD caregivers do seem to experience high levels of psychological suffering and specific problems related to their phase in life.
\end{abstract}




\subsection{Introduction}

Relatively little is known about the group of young-onset dementia (YOD) caregivers, who care for relatives who have developed dementia before the age of 65 . Since in most European countries like the Netherlands, health care services are designed to support caregiving for dementia patients at home as long as possible ${ }^{1}$, it is important to know the specific characteristics of YOD and how these affect caregivers, in order to plan care.

Numerous studies have demonstrated that caring for a person with dementia at home can have adverse effects on the psychological and physical health of the informal caregiver ${ }^{1-6}$. Informal caregivers run a higher risk of depression ${ }^{7}$, have higher levels of stress hormones ${ }^{6}$ and report lower global levels of health compared with non-caregivers ${ }^{8}$. Risk factors for these adverse effects are: a lack of social support $^{9}$, illness severity ${ }^{10}$, ADL disability ${ }^{11,12}$, and - most important of all- the patient's problem behavior ${ }^{3,6}, 9,13,14$.

YOD is not as prevalent as late onset dementia (LOD) (5\% of all dementia cases), but it can have a higher impact on patients and their families. YOD patients are faced with losing their active roles in society and seeing their plans for the future disrupted. They may still be employed or caring for their (young) children. Furthermore, YOD has a different clinical manifestation. It has been identified as being characterized more by neuropsychiatric symptoms, such as alterations in socio-emotional behavior and insight ${ }^{15}$, due to the higher prevalence of frontotemporal dementia. This may put YOD caregivers at higher risk of being adversely affected. In addition, YOD is more difficult to recognize, and on average diagnosis takes substantially longer than in the case of $\mathrm{LOD}^{16}$. The lower prevalence and broader differential diagnosis result in extra delay before an accurate diagnosis of YOD is made.

In order to adequately support caregivers, it is important to know the impact of YOD on patients' informal caregivers. This review systematically investigates the literature on the psychosocial impact of YOD on patients' informal caregivers, including their children. Additionally, we investigated whether there is a difference in impact between YOD and LOD on caregivers.

\subsection{Methods}

\section{Literature search}

A systematic literature search was performed in PubMed, Psychinfo and Cinahl in order to identify literature on the psychosocial impact of YOD on informal caregivers. For the search MESH and Thesaurus terms and free text words were used (Table 3.1), covering all publications up to October 2008. In addition, a search of the listed references in the reviewed papers was performed. Articles had to discuss informal caregivers and/or the children of people diagnosed with YOD and address the psychological/ psychosocial impact of the disease on these 
Table 3.1: Search strategy

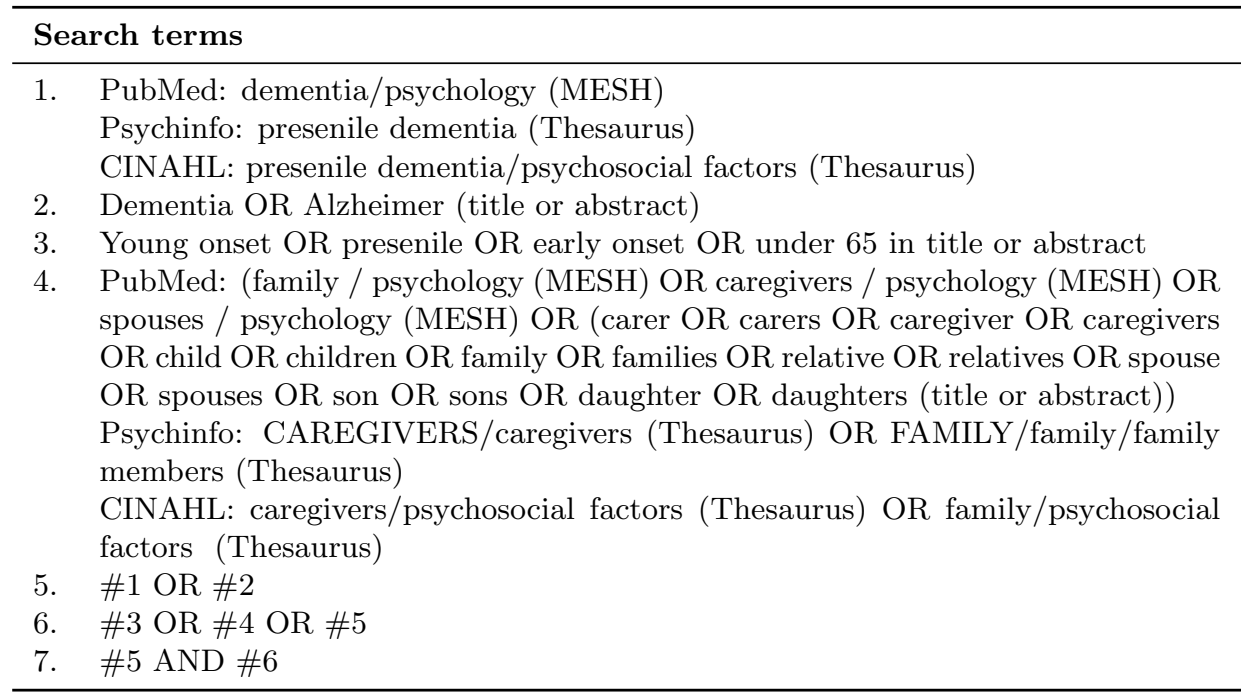

caregivers, and they had to be peer reviewed. Articles that focused on mixed groups of LOD and YOD patients, and articles that focused solely on caring for people with Huntington's disease, or on dementia after acquired brain damage, HIV, alcohol abuse or Down's syndrome, were excluded. The reason for this is that these diagnoses raise specific additional problems that will not be addressed in the present review. The search resulted in a total of 477 hits after the deletion of duplicates.

\section{Selection of studies}

The titles of the 477 remaining studies were assessed by one reviewer (DvV) and in case of doubt the abstract was read. Four hundred forty nine papers were rejected, because they did not meet the inclusion criteria. Figure 3.1 shows the selection process of the articles. The abstracts of the remaining 28 articles were assessed by two reviewers $(\mathrm{DvV}, \mathrm{MdV})$, who reached full agreement about the exclusion of a further eight articles. If the reviewers disagreed, a paper was read in full. The remaining 20 papers were read in full by one reviewer (DvV), while another reviewer read a sample of 11 papers (CB). Both reviewers reached full agreement about the inclusion or exclusion of the samples. Finally, seven articles were excluded. Cross-referencing the reference lists of the remaining articles (DvV, CB) resulted in four additional articles. Data were extracted by means of a data extraction form. 


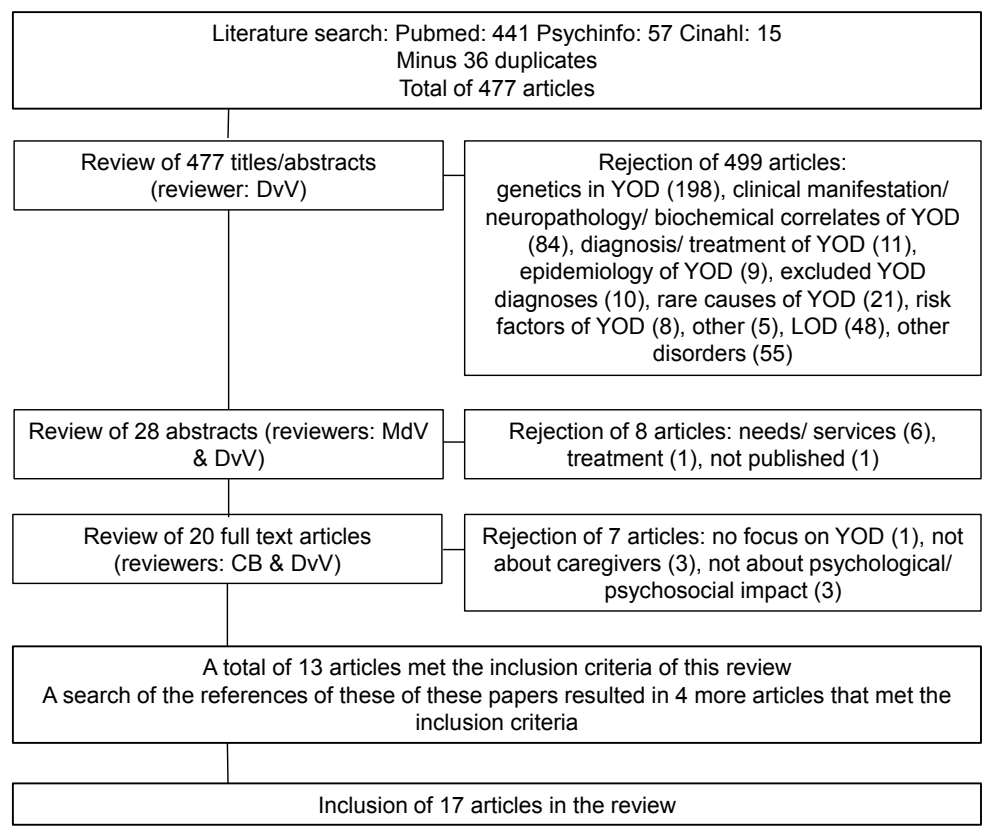

Figure 3.1: Flowchart showing the process of the selection of studies.

\section{Quality assessment}

The cross-sectional studies were qualitatively appraised by means of the quality criteria for observational studies ${ }^{17}$, and the quality checklist for qualitative studies $^{18}$ (table 3.3 and 3.4). The criteria that were not applicable for any of the articles were not included in table 3.3. One rater (DvV) assessed the quality of all articles and additionally a second rater $(\mathrm{CB})$ assessed a random sample of five articles. Inter-rater reliability was substantial, with a Cohen's kappa of 0.75. After a consensus meeting both raters reached full agreement on the quality ratings.

\subsection{Results}

\section{Methodological aspects}

The selected articles were heterogeneous in terms of design, sample sizes and methods (Table 3.2). Therefore pooling of data was not possible. One RCT (of which baseline data were of interest) was found, investigating the effects of time-limited group counseling on YOD caregivers. In addition, ten cross-sectional studies, two qualitative reviews and four case reports/expert opinions were found. 


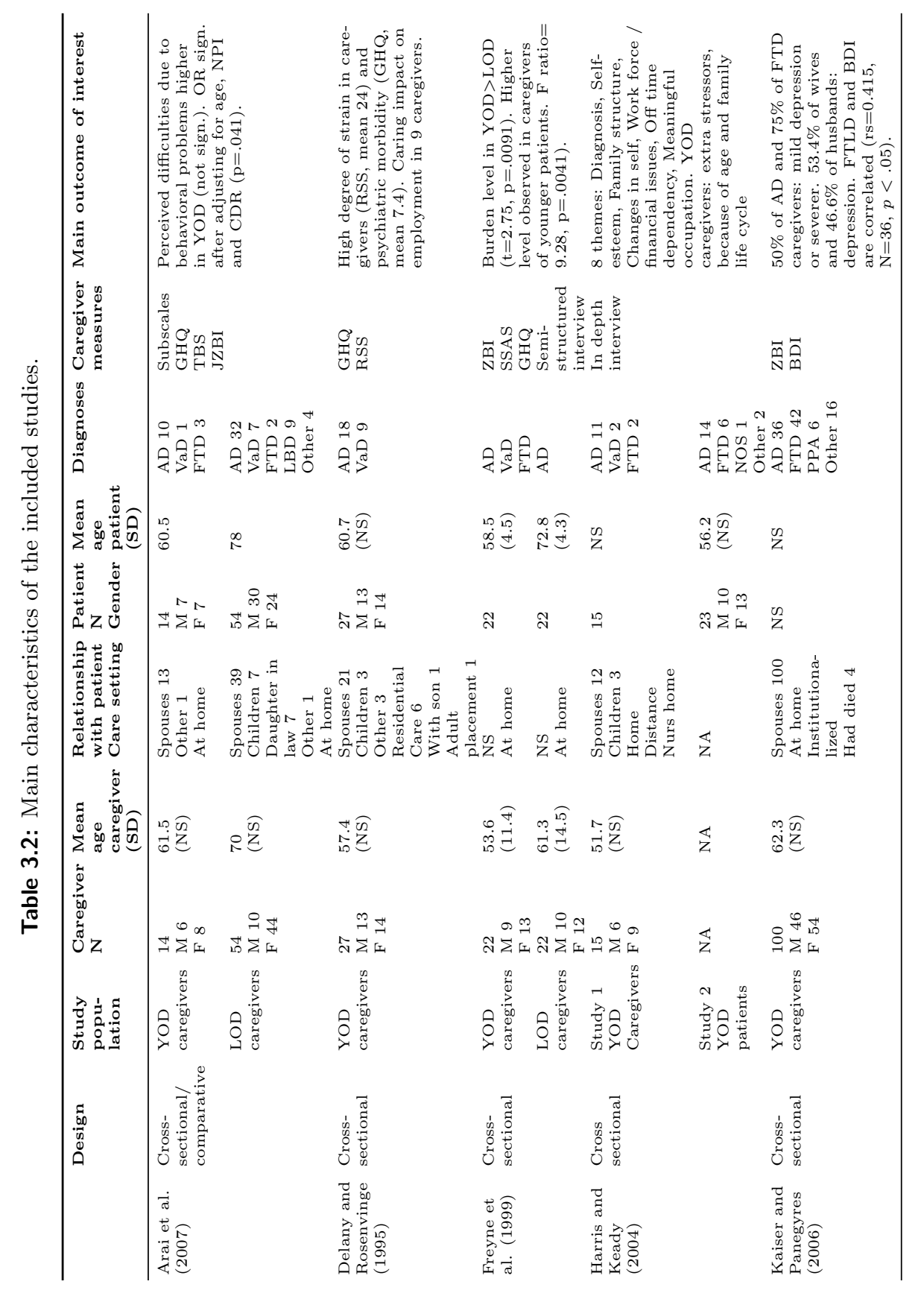




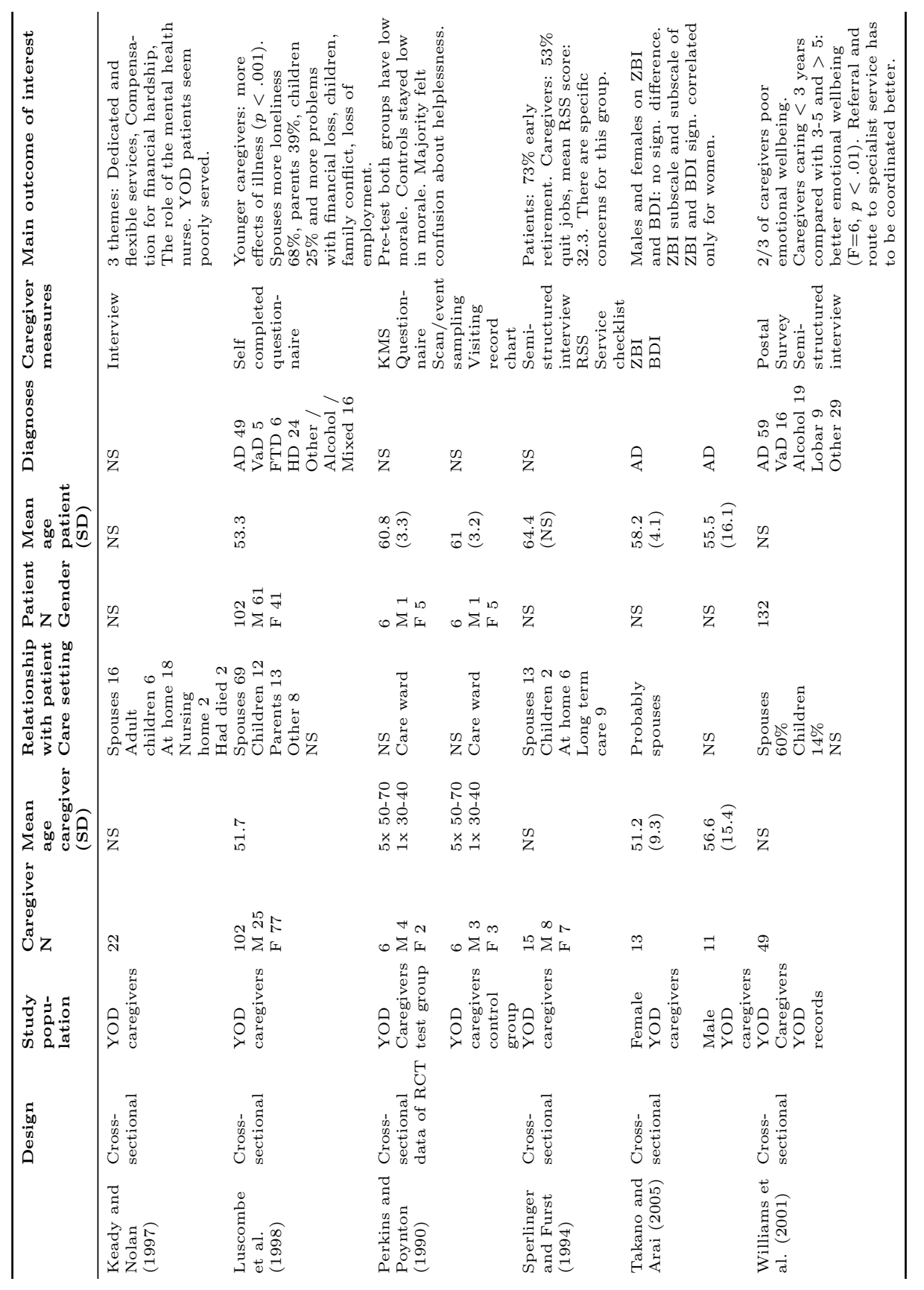


The methodological quality of the included studies varied and overall they were rated as limited. Nearly all studies had small sample sizes and only two studies compared YOD and LOD caregivers, of which one ${ }^{19}$ used groups that differed in care duration. Across studies, the sample characteristics such as age, diagnosis or relationship with patient were heterogeneous and often not fully described. The criteria that were used to define YOD differed among studies, viz. with regard to whether the patient's age was below 65 at the first hospital visit ${ }^{20}$, at the time of inclusion ${ }^{19,21-23}$, or at the time of diagnosis ${ }^{24,25}$. In four studies it was not clearly described how YOD was defined ${ }^{26-29}$.

Based on design and methodology a hierarchy of quality was composed. With regard to exploring the impact of YOD on informal caregivers, the studies of Arai et al. (2007), Luscombe et al. (1998) and Delany and Rosenvinge (1995) had the highest methodological quality, with the largest proportion of quality criteria met (Table 3.3 and 3.4). With regard to making inferences about YOD compared to LOD, the studies of Arai et al.(2007) and Freyne et al. (1999) had the highest quality. The other cross-sectional studies were of lower quality due to the limited description of their methods and results.

Some studies were limited in terms of their informative value for the present review. The relevant information in one review ${ }^{30}$ was based on an article that has been included in the present review ${ }^{23}$. In the case of the other review hardly any peer-reviewed scientific papers on the impact of YOD on caregivers were included $^{31}$. Two case studies addressed the negative impact of YOD on family members indirectly ${ }^{32}, 33$, by describing the impact of a chronic illness on a spouse or on children. The case reports and reviews were only included for background knowledge, but not presented in table 3.2, nor included in the discussion.

\section{Quantitative outcomes}

Overall, YOD caregivers were found to suffer from high levels of burden and depression. When compared with LOD caregivers, YOD caregivers experienced higher levels of burden ${ }^{19}, 20$, but this was statistically significant in only one study. This study did not find any relationship between cognitive function or behavior disturbance and caregiver burden. The other study ${ }^{20}$ found a non-significant difference in burden between YOD and LOD, but found greater perceived difficulties due to behavioral problems in YOD caregivers. Furthermore, behavioral disturbances were positively correlated with caregiver burden in both groups, whereas this was the case for illness severity only in the LOD group. Psychiatric and somatic symptoms in YOD caregivers as well as their perceived level of social support were also measured in several studies. Psychiatric symptoms were high in these studies ${ }^{19}, 20,22$, but when compared they were not significantly higher than in the case of LOD caregivers ${ }^{19}, 20$. Somatic symptoms were lower in YOD caregivers and they also reported lower levels of social support, but again these differences were not statistically significant ${ }^{19,20}$. 
Table 3.3: Quality assessment of observational cross-sectional studies.

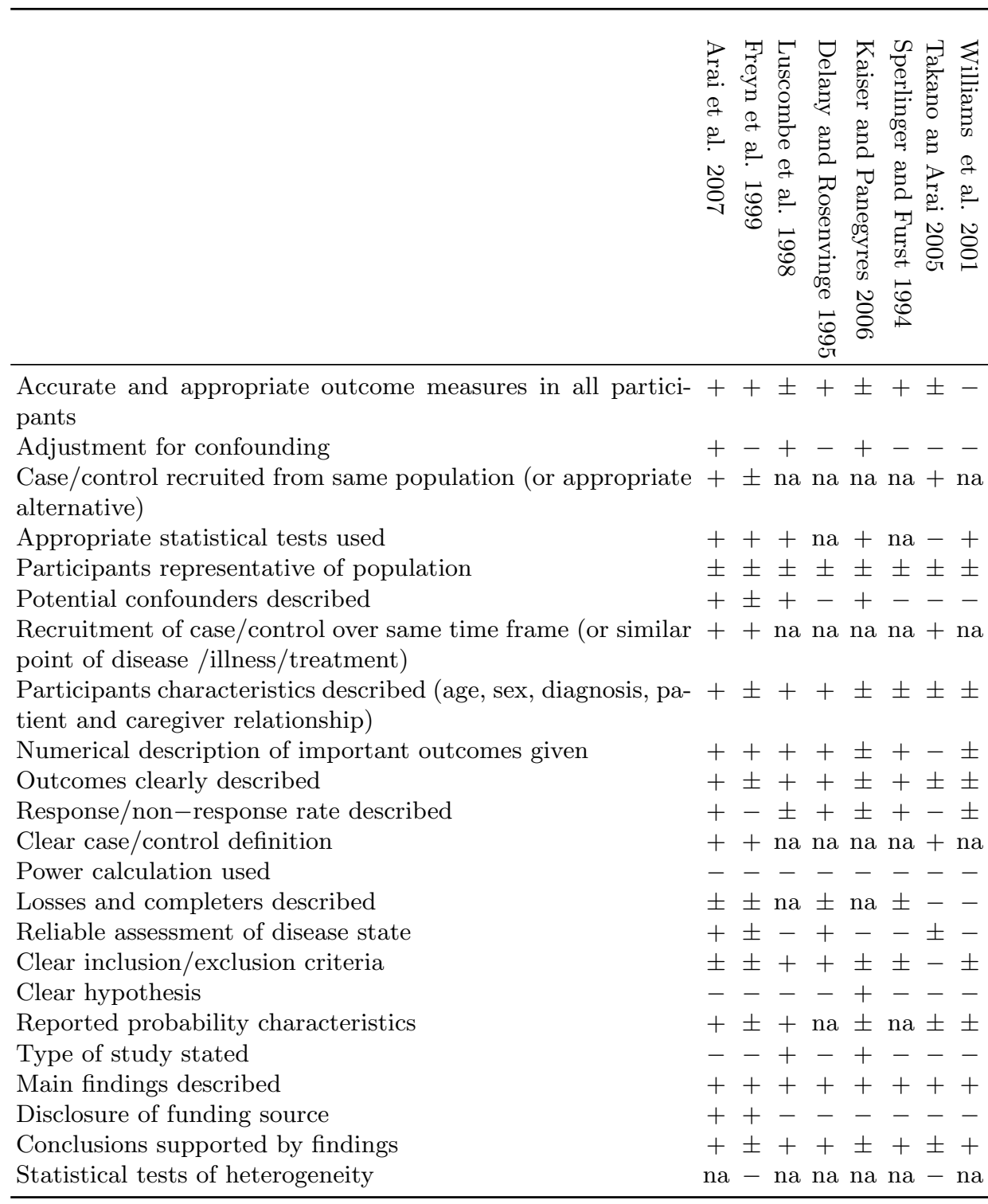

+ , criterion met; \pm , criterion partly met; -, criterion unmet; na, not applicable. 
Table 3.4: Quality assessment of qualitative cross-sectional studies.

\begin{tabular}{|c|c|c|}
\hline & $\begin{array}{l}\text { Harris and } \\
\text { Keady } 2004\end{array}$ & $\begin{array}{l}\text { Keady and } \\
\text { Nolan } 1997\end{array}$ \\
\hline $\begin{array}{l}\text { Clear statement of, and rationale for, research ques- } \\
\text { tion/aims/purposes }\end{array}$ & + & + \\
\hline $\begin{array}{l}\text { Study thoroughly contextualized by existing litera- } \\
\text { ture }\end{array}$ & + & \pm \\
\hline $\begin{array}{l}\text { Method/design apparent, and consistent with re- } \\
\text { search intent }\end{array}$ & \pm & \pm \\
\hline Data collection strategy apparent and appropriate & + & + \\
\hline Sample and sampling method appropriate & \pm & - \\
\hline Analytic approach appropriate & \pm & \pm \\
\hline $\begin{array}{l}\text { Context described and taken account of in interpre- } \\
\text { tation }\end{array}$ & - & - \\
\hline Clear audit trail given & \pm & - \\
\hline Data used to support interpretation & + & \pm \\
\hline Researcher reflexivity demonstrated & - & - \\
\hline Demonstration of sensitivity to ethical concerns & - & - \\
\hline Relevance and transferability evident & \pm & \pm \\
\hline
\end{tabular}

+ , criterion met; \pm , criterion partly met; - , criterion unmet.

In non-comparative studies high degrees of stress were found in YOD caregivers ${ }^{22,24}$, where scores were above the cut-off for psychiatric morbidity ${ }^{34}$. The majority of YOD caregivers experienced depression, ranging from mild to severe. Fifty per cent of YOD Alzheimer (AD) caregivers and $75 \%$ of YOD Frontotemporal (FTD) caregivers experienced at least mild depression ${ }^{26}$. Mild to moderate depression scores were also found in YOD caregivers in the study of Takano and Arai (2005). Kaiser and Panegyres (2006) found a slightly higher percentage of female caregivers $(53.4 \%)$ suffering from depressive symptoms than male caregivers (46.6 \%). Takano and Arai (2005) did not find significant differences between males and females on burden and depression scales, but they did identify correlations between burden subscales and mean depression score in the case of women. Patient's behavioral disturbances and cognitive functioning did not correlate with any of these subscales. The authors concluded that female YOD caregivers suffered from higher levels of burden than males. The $\mathrm{RCT}^{27}$ showed that YOD caregivers of institutionalized patients had a low morale at baseline and that time-limited group counseling had a positive effect on morale. Morale correlates positively with well-being. The authors stated that the counseling was not specific for the YOD group and could be extended to LOD caregivers. 


\section{Qualitative outcomes}

Several studies reported on the negative psychological and emotional effects of YOD on caregivers, such as feelings of frustration, grief or guilt, loneliness or social isolation, and poor emotional well-being $21,23,25$. Female YOD caregivers and younger caregivers appeared to be at higher risk for adverse psychological effects. Spousal caregivers were more at risk of experiencing loneliness compared with other YOD caregivers, such as parents or children ${ }^{23}$.

Emotional problems were commonly mentioned as a result of a changing marital relationship. For example, caregivers experienced trouble with responding to their spouse in the right way, they experienced feelings of sexual frustration, or difficulties with forming new relationships, with accompanying feelings of guilt, or with issues of selfhood and self-esteem ${ }^{25,29}$. Changes within the family structure were a common theme as well ${ }^{23,25}$. Family conflict as a result of dementia, for example, was acknowledged by $41 \%$ of YOD caregivers in the study conducted by Luscombe et al. (1998). Fifty one per cent of the caregivers reported that their children had been in conflict with their ill parent. This occurred more often with an affected father than with a mother, and was more common in the case of a relatively young parent. A patient's young age was also associated with children experiencing difficulties at school.

Furthermore, most studies reported that YOD had an impact on workforce participation and finances both for patients as well as caregivers ${ }^{22-25,29}$. In the majority of caregivers, the illness had an effect on their employment, resulting in retirement or reduced working hours ${ }^{22-24}$. Problems at work were recurrently found to lead to financial difficulties and could also lead to a lack of meaningful occupation in caregivers ${ }^{25}$.

As expected, the diagnostic process was an important theme as well ${ }^{21,23-25}$, with reports on the difficulties in obtaining a diagnosis, problems with the referral period and distress experienced by YOD caregivers due to the delay. The lack of designated services for YOD patients was also distressing for caregivers ${ }^{25}, 29$. The caregivers felt angry and guilty to be forced to accept a service intended for older people. In another study most caregivers found the services they received adequate $^{24}$, although they also gave recommendations about what services they would have liked, such as specialized daycare service for younger people with dementia.

Two case studies ${ }^{35,36}$ and reviews ${ }^{30,31}$ were in line with the notion that YOD patients and their caregivers are a specific group, with specific age-related needs. The same themes that have already been mentioned emerged from these articles, but also issues such as genetic implications, changing future expectations, a disruption of the life course, changes in and the loss of roles and a lack of social support were mentioned. Recommendations were made for support and specialist services for YOD patients and their caregivers. 


\subsection{Discussion}

Results indicate that YOD caregivers experience high levels of burden and suffer from depressive symptoms. In addition, they appear to experience a considerable number of psychosocial problems, including relational difficulties, family conflict, employment and financial issues, and negative experiences regarding the diagnostic process. However, when YOD caregivers were directly compared with LOD caregivers, the results were less conclusive. While comparative studies found higher levels of burden in YOD caregivers, only Freyne et al. (1999) found a significant difference. This significant difference is hard to interpret, because of the longer care duration of the YOD group, as longer care duration is associated with higher burden levels among caregivers of elderly people ${ }^{37}$. The fact that Arai et al. (2007) did not find a significant difference in burden contradicts the result of their study that YOD caregivers experienced greater difficulties due to behavioral problems, which is known to result in higher levels of caregiver distress ${ }^{13}$. A possible explanation could be the small sample size or the fact that patient's age at first hospital visit was used to distinguish between YOD and LOD.

The results of the present review are difficult to compare with literature on LOD caregivers, but depression levels as found by Kaiser and Panegyres (2006) viz. 50 and $75 \%$ in $\mathrm{AD}$ and FTD caregivers respectively, seem to be higher than prevalence rates in LOD caregivers viz. $22 \%^{7}$. However, high stress levels in YOD caregivers, reaching mean RSS scores of $32.3^{24}$ and $24^{22}$, have also been reported in the case of LOD caregivers, with a mean score of 27.2 reported in the study by Greene et al. $(1982)^{38}$.

Some types of age-related difficulties experienced by YOD caregivers seem specific for this group ${ }^{23}$. Work-related problems, financial problems, and problems with children occur less in the case of LOD caregivers. Furthermore, distress due to the delay in referral and diagnostic uncertainty also seems to be more specific for this group ${ }^{15,39}$. These reports underline the need for a faster diagnosis and more adequate help in this period, which may decrease caregiver distress. A lack of designated services is also a specific theme for YOD, although this was not always reported as distressing for caregivers.

The results of this review should be interpreted with caution, because of the limitations of the studies included. These studies were only based on crosssectional data, so no inferences can be drawn on the different stages of dementia. Comparative studies were few and still had several limitations. The non comparative studies did not take specific sources of caregiver distress into account e.g. illness severity, ADL disabilities or problem behavior. The studies that did examine (some of) these factors found contradicting results, which could be due to the different operationalization. Moreover, they did not make differentiations into behavioral subtypes. Furthermore, almost all studies had small sample sizes and the heterogeneity in design, methods, and sample characteristics did not allow for fair comparisons between results. In addition, there is no universally accepted tool to assess observational studies, but we applied the most commonly used quality 
criteria. It also should be noted that publication bias could have influenced the results of this review, as negative studies (finding no differences between YOD and LOD) are less likely to be published.

In conclusion, high-quality research on the difference in impact between YOD and LOD caregivers is scarce, so no firm conclusions could be drawn. Studies investigating the impact of YOD on children have not yet been conducted. Recommendations for future research include cohort studies that compare functioning and experiences of YOD caregivers with LOD caregivers during the course of the illness, taking into account dementia subtype, illness severity, sub clusters of neuropsychiatric symptoms, care setting and using the same outcome measures. 


\section{References}

1. Schneider J, Murray J, Banerjee S, Mann A. EUROCARE: a cross-national study of co-resident spouse carers for people with Alzheimer's disease: I--Factors associated with carer burden. International Journal of Geriatric Psychiatry 1999;14:651661.

2. Schulz R, O'Brien AT, Bookwala J, Fleissner K. Psychiatric and physical morbidity effects of dementia caregiving: prevalence, correlates, and causes. The Gerontologist 1995;35:771-791.

3. Baumgarten M, Battista RN, Infante-Rivard C, Hanley JA, Becker R, Gauthier S. The psychological and physical health of family members caring for an elderly person with dementia. Journal of Clinical Epidemiology 1992;45:61-70.

4. Kiecolt-Glaser JK, Dura JR, Speicher CE, Trask OJ, Glaser R. Spousal caregivers of dementia victims: longitudinal changes in immunity and health. Psychosomatic Medicine 1991;53:345-362.

5. Pinquart M, Sorensen S. Differences between caregivers and noncaregivers in psychological health and physical health: a meta-analysis. Psychology and Aging 2003;18:250-267.

6. de Vugt ME, Nicolson NA, Aalten P, Lousberg R, Jolle J, Verhey FRJ. Behavioral Problems in Dementia Patients and Salivary Cortisol Patterns in Caregivers. Journal of Neuropsychiatry \&5 Clinical Neurosciences 2005;17:201-207.

7. Cuijpers P. Depressive disorders in caregivers of dementia patients: a systematic review. Aging \& Mental Health 2005;9:325-330.

8. Vitaliano PP, Zhang J, Scanlan JM. Is caregiving hazardous to one's physical health? A meta-analysis. Psychological Bulletin 2003;129:946-972.

9. Chang BL, Brecht ML, Carter PA. Predictors of social support and caregiver outcomes. Women \& Health 2001;33:39-61.

10. Lieberman MA, Fisher L. The impact of chronic illness on the health and well-being of family members. The Gerontologist 1995;35:94-102.

11. Razani J, Kakos B, Orieta-Barbalace C, et al. Predicting caregiver burden from daily functional abilities of patients with mild dementia. Journal of the American Geriatrics Society 2007;55:1415-1420.

12. Nagatomo I, Akasaki Y, Uchida M, Tominaga M, Hashiguchi W, Takigawa M. Gender of demented patients and specific family relationship of caregiver to patients influence mental fatigue and burdens on relatives as caregivers. International Journal of Geriatric Psychiatry 1999;14:618-625.

13. Ballard C, Lowery K, Powell I, O'Brien J, James I. Impact of behavioral and psychological symptoms of dementia on caregivers. International Psychogeriatrics 2000;12:93-105.

14. de Vugt ME, Riedijk SR, Aalten P, Tibben A, van Swieten JC, Verhey FR. Impact of behavioural problems on spousal caregivers: a comparison between Alzheimer's disease and frontotemporal dementia. Dementia and Geriatric Cognitive Disorders 2006;22:35-41. 
15. Mendez MF. The accurate diagnosis of early-onset dementia. International Journal of Psychiatry in Medicine 2006;36:401-412.

16. Van Vliet D, De Vugt ME, Koopmans RTCM, Bakker C, Verhey FRJ. How do caregivers of patients with early onset dementia perceive the period prior to diagnosis. (Submitted).

17. Mallen C, Peat G, Croft P. Quality assessment of observational studies is not commonplace in systematic reviews. Journal of Clinical Epidemiology 2006;59:765769 .

18. Walsh D, Downe S. Appraising the quality of qualitative research. Midwifery 2006;22:108-119.

19. Freyne A, Kidd N, Coen R, Lawlor BA. Burden in carers of dementia patients: higher levels in carers of younger sufferers. International Journal of Geriatric Psychiatry 1999;14:784-788.

20. Arai A, Matsumoto T, Ikeda M, Arai Y. Do family caregivers perceive more difficulty when they look after patients with early onset dementia compared to those with late onset dementia? International Journal of Geriatric Psychiatry 2007;22:1255-1261.

21. Williams T, Cameron I, Deardon T. From pillar to post-a study of younger people with dementia. Psychiatric Bulletin 2001;25:384-387.

22. Delany N, Rosenvinge H. Presenile dementia: Sufferers, carers and services. International Journal of Geriatric Psychiatry 1995;10:597-601.

23. Luscombe G, Brodaty H, Freeth S. Younger people with dementia: Diagnostic issues, effects on carers and use of services. International Journal of Geriatric Psychiatry 1998;13:323-330.

24. Sperlinger D, Furst M. The service experiences of people with presenile dementia: A study of carers in one London borough. International Journal of Geriatric Psychiatry 1994;9:47-50.

25. Harris PB, Keady J. Living with early onset dementia: exploring the experience and developing evidence-based guidelines for practice. Alzheimer's Care Quarterly 2004;5:111-122.

26. Kaiser S, Panegyres PK. The psychosocial impact of young onset dementia on spouses. American Journal of Alzheimer's Disease and Other Dementias 2006;21: 398-402.

27. Perkins RE, Poynton CF. Group counselling for relatives of hospitalized presenile dementia patients: a controlled study. The British Journal of Clinical Psychology 1990;29 ( Pt 3):287-295.

28. Takano M, Arai H. Gender difference and caregivers' burden in early-onset Alzheimer's disease. Psychogeriatrics 2005;5:73-77.

29. Keady J, Nolan M. Raising the profile of younger people with dementia. Mental Health Nursing 1997;17:7-10.

30. Diehl J, Forstl H, Jansen S, Kurz A. Frontotemporal dementia: specific problems for caregivers. Zeitschrift fur Gerontologie und Geriatrie 2004;37:301-306. 
31. Tindall L, Manthorpe J. Early onset dementia: a case of ill-timing? Journal of Mental Health 1997;6:237-249.

32. Farkas SW. Impact of chronic illness on the patient's spouse. Health $\mathcal{E}$ Social Work 1980;5:39-46.

33. Liebson E, Rauch P, Graff S, Folstein M. Early-onset dementia: diagnostic considerations and implications for families. Harvard Review of Psychiatry 2005;13:102111.

34. Ulstein I, Bruun Wyller T, Engedal K. The relative stress scale, a useful instrument to identify various aspects of carer burden in dementia? International Journal of Geriatric Psychiatry 2007;22:61-67.

35. Armstrong M. The needs of people with young-onset dementia and their carers. Journal of Professional Nursing 2003;18:681-684.

36. Williams O, Keady J, Nolan M. Younger-onset Alzheimer's disease: learning from the experience of one spouse carer. Journal of Clinical Nursing 1995;4:31-36.

37. Sugihara Y, Sugisawa H, Nakatani Y, Shibata H. Impact of duration of caregiving on stress among primary caregivers of elderly. Nippon Koshu Eisei Zasshi 1998;45:320-335.

38. Greene JG, Smith R, Gardiner M, Timbury GC. Measuring behavioural disturbance of elderly demented patients in the community and its effects on relatives: a factor analytic study. Age Ageing 1982;11:121-126.

39. Sampson EL, Warren JD, Rossor MN. Young onset dementia. Postgraduate Medical Journal 2004;80:125-139. 


\section{Chapter 4}

\section{Time to diagnosis in young-onset dementia as compared to late-onset dementia}

Based on: van Vliet D, de Vugt ME, Bakker C, Pijnenburg YAL, VernooijDassen MJFJ, Koopmans RTCM, Verhey FRJ. (2012) Time to diagnosis in youngonset dementia as compared to late-onset dementia. Psychological Medicine,1-10, doi:10.1017/S0033291712001122. 


\section{Abstract}

Background The extent to which specific factors influence diagnostic delays in dementia is unclear. Therefore, the aim of the present study is to compare duration from symptom onset to diagnosis for young-onset dementia (YOD) and late-onset dementia (LOD) and to assess the effect of age at onset, type of dementia, gender, living situation, education and family history of dementia on this duration.

Methods Data on 235 YOD-and 167 LOD-patients collected from caregivers from two prospective cohort studies were used. Multiple linear regression analysis was performed.

Results The duration between symptom onset and the diagnosis of YOD exceeded that of LOD by an average of 1.6 years (2.8 versus 4.4 years). Young age and being diagnosed with FTD were related to increases in the time to diagnosis. Subjects with vascular dementia experienced shorter time to diagnosis.

Conclusions There is a need to raise special awareness of YOD to facilitate a timely diagnosis. 


\subsection{Introduction}

The timely diagnosis of dementia is increasingly regarded as a prerequisite for beginning adequate treatment, planning for the future and coping with the prognosis ${ }^{1,2}$. However, diagnosis is often delayed until disabling symptoms are well established $^{1}$. The time from the presentation of an individual's first symptoms to a dementia diagnosis has been found to be one year on average $^{3}$, but delays of up to five years have also been reported ${ }^{4,5}$. Some of the barriers to early diagnosis in primary care are failure to recognize the slowly evolving symptoms, lack of confidence to make and disclose a diagnosis, negative attitudes towards diagnosis, for example because of a lack of support resources for the person with dementia or potential risks of an early diagnosis ${ }^{6,7}$. Long delays may be particularly true for young-onset dementia (YOD) due to its lower prevalence and larger variety of etiologies $^{8-10}$. YOD may also present differently from late onset dementia (LOD), with more marked neuropsychiatric symptoms than cognitive symptoms in frontotemporal dementia (FTD) and several other rare etiologies typically occurring at a young age $\mathrm{e}^{8,11,12}$. Non-recognition can have serious consequences for younger people and their families, such as tension with family members living in the same house (often children) and a higher chance of divorce or job loss ${ }^{13}$. Timely recognition makes it possible to adjust to the consequences of the disease; therefore, it is important to gain insight into the factors associated with time to diagnosis and to what extent they influence the duration of that period. Young age ${ }^{10,14}$ and a diagnosis of $\mathrm{FTD}^{12}$ have both been associated with diagnostic difficulties in separate studies, so their independent roles are unknown. We hypothesize that younger age is related to longer time to diagnosis independent of the influence of a diagnosis of FTD. Furthermore, it is assumed that a high education level and female gender are associated with a shorter time to diagnosis based on studies of help-seeking behaviour ${ }^{15-17}$. Likewise, it is hypothesized that a family history of dementia is related to the length of time to diagnosis ${ }^{18}$ because such a history would promote the earlier recognition of symptoms. In addition, recognition of dementia by general practitioners in patients living alone has been found to be reduced, thus we expect this to be related to increased time to diagnosis ${ }^{19}$. The aim of the present study is to compare the duration of the period from symptom onset to diagnosis for YOD and LOD and to investigate the influence of age of onset, dementia type, gender, education, living situation and family history of dementia on the time to diagnosis.

\subsection{Method}

\section{Subjects}

This study is part of a Dutch prospective cohort study, the NeedYD-study (Needs in Young onset Dementia), which has been described in more detail elsewhere ${ }^{20}$. Two hundred and fifteen participants with YOD were recruited who exhibited 
an age at onset of less than 65 years. They were included in the study together with their primary caregivers. Patients were consecutive referrals from university medical centres (56), regional hospitals (10), and regional community mental health services (20); they applied to participate (14) or were recruited through specialised day care facilities (115). The diagnoses were made on the basis of clinical, neuropsychological and neuroimaging data according to the criteria from the $4^{\text {th }}$ edition of the Diagnostic and Statistical Manual of Mental Disorders, text revision (2000) for dementia ${ }^{21}$ and the Dutch consensus guidelines ${ }^{22}$ that include internationally accepted criteria for the subtype diagnoses ${ }^{23-27}$. Clinical diagnoses were checked against clinical files for every patient and against medical hospital records when available. The exclusion criteria were (1) unreliability on the part of the informant (if the informant and person with dementia did not have contact with each other at least once a week), (2) lack of informed consent of the participant, (3) living in a nursing home or (4) dementia caused by HIV, traumatic brain injury, Down's syndrome, Huntington's chorea or alcohol dementia. Since these diagnoses are associated with a range of specific problems, which would have obscured the impact of a younger age at dementia onset, these diagnoses were excluded.

One-hundred-and-seventy-three subjects with LOD were used as a comparison group. They were selected from a 2-year follow-up cohort study, the MAASBED study (the Maastricht Study of Behaviour in Dementia) ${ }^{28}$, overall using the same design, assessment measures and diagnostic criteria. The NeedYD-study protocol was based on the methods of the MAASBED-study to be able to compare outcomes. Both the MAASBED -and NeedYD-study are prospective studies, with assessments every six months during two years. Inclusion criteria and recruitment differed somewhat between studies. In the MAASBED study, patients with dementia were included irrespective of their diagnostic subtype and they were consecutively enrolled from the Memory Clinic of the MUMC+ or from the Regional Institute for Community Mental Health Care (RIAGG) in Maastricht. The MAASBED cohort included 26 YOD patients added to the YOD group in the present study. For the current study, the data collected from the primary caregivers of the patients in the NeedYD - and MAASBED-study were used.

\section{Procedure}

The study protocol was approved by the Medical Ethics Committee of the University Medical Centre Maastricht. The written informed consent of the subjects or their legal representatives was obtained prior to the investigation. During a semi-structured interview with the patient as well as the primary caregiver the following questions were asked: 'Which diagnosis was given to you? Do you know what type of dementia you have?', followed by the question: 'In which year did the very first symptoms or complaints occur'. The interviewer was instructed to ask whether there were any earlier signs or symptoms. In addition, a semi-structured open-ended interview with the primary caregiver was tape-recorded including the 
following questions: 'When did you first notice something was wrong with your family member? What did you notice?' 'Can you describe the period prior to diagnosis?' 'What did you think was wrong?' 'Which problems did you experience during this period?' Again the interviewer was instructed to encourage caregivers to elaborate on their answers and to think of any other signs or symptoms that occurred. The year of the earliest sign or symptom, either in the cognitive, behavioural or functional domain was recorded as the year of symptom onset. The moment that caregivers consciously noticed changes mostly occurred later than the moment that was indicated with hindsight. In these cases, the year that was indicated with hindsight was noted as the year of symptom onset. Subjects' medical records were used to determine the year in which each diagnosis was established. The duration (in years) between symptom onset and diagnosis was calculated by subtracting the year of symptom onset from the year of diagnosis. Demographic data were collected that indicated age, gender, education level divided into eight categories of the Dutch educational system ranging from (1) primary school to (8) the completion of university-level education and family history of dementia, which was determined to exist if any family member had had dementia. The Global Deterioration Scale (GDS) was used to assess the severity of the dementia ${ }^{29}$.

\section{Analysis}

First a sample-size calculation was performed for the group comparisons and the regression-analysis, with a medium effect size, an alpha level of .05 and power of .80 , which showed that the sample size should be at least 64 in each group for the group comparisons and 103 for the regression analysis. Analyses were performed using the Statistical Package for Social Sciences (SPSS) software, version 17.0. To assess descriptive group characteristics Chi square tests and Mann-Whitney U tests were used as appropriate. For comparisons of duration to diagnosis between groups (YOD and LOD) Mann-Whitney U tests were performed. These nonparametric tests were used, because the distribution of the dependent variable was skewed or because the variance of the dependent variable was not homogeneous across groups. A multiple linear regression analysis was performed to assess the relationship between group (YOD vs. LOD), type of dementia, living arrangements (alone vs. not alone), education, gender and family history of dementia (the independent variables) and the estimated duration from symptom onset to diagnosis in years (the dependent variable). The data were log-transformed because of the heteroscedastic variance of the residuals of the variable: duration from symptom onset to diagnosis. The types of dementia were divided into 4 categories and dummy-coded with Alzheimer's disease (AD) as the reference group: (1) AD, (2) frontotemporal dementia (FTD), (3) vascular dementia (VaD) and mixed dementia (AD and $\mathrm{VaD}$ ) and (4) Other. Education was recoded as low (1-4) or high (5-8). The collinearity between the predictors was explored using phi-correlations. The potential influence of outliers was determined. In order to detect outliers, which are data points of the dependent variable (i.e. time to diagnosis) that differ 
largely from the values predicted by the model, the standardized residual (residual/estimated standard deviation of the residual) were assessed. As a general rule $99.9 \%$ of standardized residuals (or z-scores) should lie between -3.29 to 3.29 and 3 is generally used as an approximation. The alpha level was set at .05 for the analyses. In addition, outliers were detected by visual inspection of the data. For these cases it was checked whether the year of symptom onset that was noted by the researcher corresponded with the tape-recorded interviews. In one case, this resulted in correction of the year of symptom onset.

\subsection{Results}

\section{Sample characteristics}

The overall study group consisted of 414 patients (241 with YOD and 173 with LOD). Information on the duration between symptom onset and diagnosis was not available for 6 YOD and 6 LOD patients (year of diagnosis was missing in 1 case, year of symptom onset in 11 cases). These patients were similar to the group with complete data with regard of age at study entry $(\mathrm{U}=2291.5, \mathrm{p}=.78)$, gender ( $\mathrm{p}=.25$, Fisher's exact test $)$, education ( $\mathrm{p}=.47$, Fisher's exact test $)$, distribution of diagnoses $\left(\mathrm{p}=.96\right.$, Fisher's exact test) and group (YOD vs. LOD) $\left(\chi^{2}=.34, p=\right.$ $.56)$. Thus, 402 patients with complete data were included in this study. The mean estimated age at onset was for the YOD group 54.8 years $(\mathrm{SD}=5.9)$ and for the LOD group 75.8 years $(\mathrm{SD}=5.9)$. Table 1 shows the age at diagnosis and the sample characteristics of both groups at study entry. The patients of the YOD group were more frequently male, they had a higher education and they more often had a family member with dementia. In addition there were significant differences in the distribution of diagnoses between groups, with more FTD and 'Other' dementias in the YOD group. The dementia was more severe in the YOD group based on GDS-scores. MMSE-scores were missing in a large sample and therefore less reliable. Spouses were most often the primary caregiver in the YOD group, while in LOD children were primary caregiver in the largest proportion of cases. In the LOD group there were also more patients living alone than in the YOD group.

\section{Duration from symptom onset to diagnosis}

The group comparison between YOD and LOD of estimated duration from symptom onset to diagnosis showed a significant difference with an average time to diagnosis of 4.4 years $(S D=3.1$; range $0.5-18.0)$ in the YOD group and an average time to diagnosis of 2.8 years $(S D=2.1$; range $0.2-10.0)$ in the LOD group $(U=13343.5, p<.001)$. For each dementia subtype, the period was longer in the YOD group than in the LOD group, with significant differences for those with AD $(U=6212.5, p<.001)$ and $\operatorname{VaD}(U=353.0, p=.006)$. Differences between young 
Table 4.1: Demographic variables at study-entry of the YOD and LOD group.

\begin{tabular}{|c|c|c|c|c|}
\hline & $\begin{array}{l}\text { YOD } \\
(\mathrm{N}=\mathbf{2 3 5})\end{array}$ & $\begin{array}{l}\text { LOD } \\
(\mathrm{N}=167)\end{array}$ & Test value & $p$ value \\
\hline Age at diagnosis, mean $\pm \mathrm{SD}$ & $59.2 \pm 5.3$ & $78.6 \pm 5.7$ & & \\
\hline Age at study-entry, mean $\pm \mathrm{SD}$ & $61.6 \pm 5.3$ & $78.6 \pm 5.7$ & & \\
\hline Sex, $\%$ Female & 44.7 & 61.7 & $\chi^{2}(1)=11.3$ & .001 \\
\hline \multicolumn{5}{|l|}{ Education, $\%$ a } \\
\hline Low (level 1,2) & 44.3 & 67.1 & $\chi^{2}(2)=23.9$ & $<.001$ \\
\hline Medium (level 3-5) & 34 & 25.1 & & \\
\hline High (level 6-8) & 21.7 & 7.8 & & \\
\hline \multicolumn{5}{|l|}{ Diagnosis, $\mathrm{N}^{\mathrm{b}}$} \\
\hline $\mathrm{AD}$ & 139 & 122 & $\chi^{2}(3)=24.4$ & $<.001$ \\
\hline FTD & 29 & 3 & & \\
\hline \multicolumn{5}{|l|}{$\mathrm{VaD}$} \\
\hline $\mathrm{VaD}$ & 27 & 27 & & \\
\hline Mixed & 8 & 6 & & \\
\hline \multicolumn{5}{|l|}{ Other } \\
\hline PA & 14 & 3 & & \\
\hline DLB & 7 & 2 & & \\
\hline PDD & 1 & 4 & & \\
\hline Alcohol & 1 & - & & \\
\hline MELAS & 1 & - & & \\
\hline TE & 1 & - & & \\
\hline MSA & 1 & - & & \\
\hline Uncertain & 6 & - & & \\
\hline Family history of dementia, $\%$ & $\begin{array}{l}49.6 \\
(\mathrm{n}=232)\end{array}$ & $\begin{array}{l}39.1 \\
(\mathrm{n}=156)\end{array}$ & $\chi^{2}(1)=4.1$ & .042 \\
\hline Living alone, $\%$ & 8.9 & 40.1 & $\chi^{2}(1)=55.5$ & $<.001$ \\
\hline \multicolumn{5}{|c|}{ Caregiver relationship with patient, $\%$} \\
\hline Spouse & 88.5 & 41.3 & $\chi^{2}(2)=103.4$ & $<.001$ \\
\hline Child & 6.8 & 44.3 & & \\
\hline Other & 4.7 & 14.4 & & \\
\hline \multicolumn{5}{|l|}{ Dementia severity (GDS-score), \% } \\
\hline Mild (score 3,4) & 58.7 & 71.3 & $\chi^{2}(2)=19.7$ & $<.001$ \\
\hline Moderate (score 5) & 26.7 & 26.9 & & \\
\hline Severe (score 6,7 ) & $\begin{array}{l}14.7 \\
(\mathrm{n}=225)\end{array}$ & 1.8 & & \\
\hline MMSE-score, mean $\pm \mathrm{SD}^{\mathrm{c}}$ & $\begin{array}{l}21.1 \pm 6.6 \\
(n=172)\end{array}$ & $\begin{array}{l}18.1 \pm 4.6 \\
(\mathrm{n}=161)\end{array}$ & $\mathrm{U}=9038.5$ & $<.001$ \\
\hline
\end{tabular}

Note: PA: progressive aphasia, DLB: dementia with Lewy bodies, PDD: Parkinson's Disease Dementia, Alcohol: alcohol related dementia, TE: toxic encephalopathy, MSA: multiple system atrophy, Uncertain: dementia of uncertain origin. a Education level ranged from the categories (1) primary school to (8) the completion of university-level education. b Based on the categories: AD=Alzheimer's disease; FTD = Frontotemporal dementia, $\mathrm{VaD}=$ Vascular dementia and mixed dementia, Other=Other dementias. ${ }^{\mathrm{c}}$ Reasons for missing MMSE-scores: refusal, cognitive problems e.g. language problems, emotional reactions or lack of motivation during testing. 
Table 4.2: Estimated duration (years) from symptom onset to diagnosis for each dementia subtype and test-statistics for the differences between young and late onset AD and $\mathrm{VaD}$.

\begin{tabular}{lrrrrrr}
\hline Diagnosis & \multicolumn{2}{c}{ YOD } & \multicolumn{2}{c}{ LOD } & \multicolumn{2}{c}{ Total } \\
& Mean (sd) & N & Mean (sd) & N & Mean (sd) & N \\
\hline All & $4.4(3.1)$ & 235 & $2.8(2.1)$ & $167^{\text {a }}$ & $3.8(2.8)$ & 402 \\
AD & $4.2(3.0)$ & 139 & $3.0(2.2)$ & $122^{\mathrm{b}}$ & $3.6(2.7)$ & 261 \\
FTD & $6.4(3.6)$ & 29 & $3.3(2.1)$ & 3 & $6.1(3.5)$ & 32 \\
VaD & $3.9(2.7)$ & 35 & $2.2(1.8)$ & $33^{\mathrm{c}}$ & $3.1(2.4)$ & 68 \\
Other & $4.1(3.3)$ & 32 & $3.4(1.3)$ & 9 & $4.0(3.0)$ & 41 \\
\hline
\end{tabular}

AD, Alzheimer's disease; FTD, Frontotemporal dementia; VaD, Vascular and mixed dementia. ${ }^{\mathrm{a}} U=13343.5, p<.001{ }^{\mathrm{b}} U=6212.5, p<.001{ }^{\mathrm{c}} U=353.0, p=.006$.

Table 4.3: Results from the multiple linear regression analysis.

\begin{tabular}{lrrrr}
\hline & B & SE B & $\beta$ & $\mathbf{t}$ \\
\hline Constant & 1.60 & 0.34 & & 4.71 \\
Group, YOD vs LOD & -0.35 & 0.09 & $-.20^{* *}$ & -3.81 \\
AD vs. FTD & 0.38 & 0.15 & $.12^{*}$ & 2.49 \\
AD vs. VaD & -0.32 & 0.12 & $-.13^{*}$ & -2.70 \\
AD vs. Other & -0.03 & 0.15 & -.01 & -0.18 \\
Sex, male vs female & -0.14 & 0.08 & -.09 & -1.71 \\
Education, low vs high & -0.00 & 0.10 & -.00 & -0.04 \\
Living arrangements, & 0.09 & 0.11 & .04 & 0.80 \\
alone vs not alone & & & & \\
\hline
\end{tabular}

AD, Alzheimer's disease; FTD, Frontotemporal dementia; VaD, Vascular and mixed dementia. Note: Adjusted $R^{2}=.10,(p<$ $.001) ; * * p<.001 ; * p<.05$.

and late onset FTD or other dementias could not be assessed because of the small sample sizes, especially in the LOD group (table 2).

\section{Factors related to time to diagnosis}

The data for the variable 'family history of dementia' were missing in 19 cases. In most of these cases, the person providing the information was not a spouse or partner and may not have been aware of the family history. This was significantly more frequent in the group with missing data than in the group with complete data (spouse vs other: $p<.001$, Fisher's exact test). To determine the effect of family history of dementia on time from symptom onset to diagnosis, a regression analysis was performed with the predictors group, type of dementia, living arrangements, gender and education in the first block and 'family history of dementia' in a second block. Adding this variable did not significantly improve the model $\Delta R^{2} \approx 0(p=$ 
.40); therefore, family history of dementia was left out of the analyses so that the cases with missing data for this variable could still be included.

The regression analysis (table 3 ) with estimated time from symptom onset to diagnosis as dependent variable and group, type of dementia, living arrangements, gender and education as predictors showed that having LOD, $\beta=-.20, t(395)=$ $-3.81, p<.001$, and being diagnosed with $\mathrm{VaD}$ as opposed to $\mathrm{AD}, \beta=-.13$, $t(395)=-2.70, p=.007$, were significantly associated with a shorter time to diagnosis and a diagnosis of FTD with a longer time to diagnosis $\beta=.12, t(395)=$ $2.49, p=.013$, with group the most important predictor. The model explained $10 \%$ of the variance.

A sensitivity analysis was performed excluding the YOD patients that were recruited in the MAASBED study which did not change the results. In addition, the analyses were repeated, excluding the FTD and 'other' diagnostic categories and results again showed that having $\operatorname{LOD} \beta=-.34, t(324)=-5.93, p<.001$ and being diagnosed with $\mathrm{VaD}$ as opposed to $\mathrm{AD} \beta=-.11, t(324)=-2.19, p=.029$ were significantly associated with a shorter time to diagnosis. There were no outliers with a standardised residual outside the range of \pm 3 . A post-hoc regression analysis of family history of YOD within the YOD group showed that this was not a significant predictor of the duration from symptom onset to diagnosis, $\beta=-.03, t(190)=-0.35, p=.73$.

\section{Dementia severity around time of diagnosis}

Post-hoc we selected patients that were included shortly after diagnosis and we excluded the FTD-subcategory to compare more homogeneous groups. Time from symptom onset to diagnosis, GDS-score and MMSE-score were compared between these subsamples of YOD $(n=93)$ and LOD patients $(n=164)$. Results confirmed our previous finding that a significantly longer time from symptom onset to diagnosis was found in the YOD group (4.6 years; $\mathrm{SD}=3.1$ ) compared with the LOD-group (2.8 years; $\mathrm{SD}=2.1)(U=4789.0, p<.001)$. Groups did not differ in GDS-scores $\left(\chi^{2}(2)=5.5, p=.065\right)$, which were categorised into mild $(74.7 \%$ YOD, $71.3 \%$ LOD), moderate (18.7\% YOD, 26.8 LOD) and severe stage dementia (6.6\% YOD, $1.8 \%$ LOD). There was a significant difference in MMSE-scores between groups, wih a mean score of $20.3(\mathrm{SD}=5.6, \mathrm{n}=85)$ in the YOD group versus $18.1(\mathrm{SD}=4.5, \mathrm{n}=158)$ in the LOD group $(\mathrm{F}(1)=10.9, \mathrm{p}=.001)$.

\subsection{Discussion}

\section{Main findings}

This study investigated the estimated time that elapses between the appearance of the first symptoms of dementia and moment of diagnosis using a relatively large sample of both YOD and LOD patients. The group comparisons showed that the duration of this period in the YOD group took an average of 1.6 years longer than 
in the LOD group. The average time from symptom onset to diagnosis in the YOD group exceeded that of the LOD group for every dementia subtype; it was statistically significant for $\mathrm{AD}$ and $\mathrm{VaD}$.

The regression analysis showed that having YOD was the most important predictor for an extended period between symptom onset and diagnosis. A diagnosis of FTD independent of age at onset was also associated with a longer time to diagnosis. These findings can be explained by the typical clinical characteristics of FTD, which include behavioural and personality changes and an early loss of insight, both of which can delay help-seeking behaviour ${ }^{12,13,30}$. Vascular dementia appeared to be diagnosed earlier in comparison to Alzheimer's disease. This trend may be a function of the more abrupt onset of the disease when it was due to cerebrovascular accidents (CVAs), the presence of neurological symptoms, or its potentially more fluctuating course ${ }^{31}$. Higher education levels and female gender were hypothesised to facilitate earlier recognition and help-seeking behaviour ${ }^{15-17}$. These effects may be masked by other factors such as the failure of physicians to recognise dementia or their providing a diagnosis other than one of dementia ${ }^{13}$. This may have resulted in a prolonged time from symptom onset to diagnosis, while presenting to services could have taken place early. Unfortunately, the moment presenting to services was not assessed in our study. Contrary to our expectations, family history of dementia and living situation did not predict time to diagnosis. The finding that living situation is not related to time to diagnosis may be explained by the small number of patients living alone in the YOD group.

\section{The age at onset effect}

Although the findings regarding time to diagnosis in YOD patients are supported by the previous literature, this period was much longer than that previously found: 3.3 years in the YO-AD group, 4.9 years in the YO-FTD group and 1 year in the LOD group ${ }^{3,12}$. This discrepancy could be a result of country-related differences or differences in the way the time between symptom onset and diagnosis was estimated. Whereas the former study ${ }^{12}$ used medical records to determine both the moment of first symptoms and the diagnosis, the latter ${ }^{3}$ based the data regarding both the moment of symptom onset and the moment of diagnosis on caregiver recollections. In the current study, the information on the moment of symptom onset was collected through caregivers and the moment of diagnosis from medical records. The most important and inevitable drawback of all these studies is the retrospective estimation of moment of symptom onset, which alone could account for heterogeneous study outcomes.

Dementia is a slowly progressing condition, in which pathological changes generally occur over extended periods before clinical presentation ${ }^{32}$. The fact that it takes time to recognize symptoms and diagnose them is therefore an inherent feature of many dementia aetiologies. There are several possible explanations for the longer time between estimated symptom onset and diagnosis in YOD. The finding that disease severity at time of diagnosis was comparable between groups 
indicates that YOD caregivers may notice the dementia symptoms in an earlier stage of the disease process. This may be due to the higher demands and expectations of younger people, for example at work. However, when it concerns VaD, one may expect symptoms to be picked up by YOD and LOD caregivers in a roughly similar stage of the disease process, because of the more obvious signs of VaD. Still, for the YO-VaD patients in the present study the time from symptom onset to diagnosis was substantially longer than in the LO-VaD group. Previous studies showed equivocal results. Similar or higher MMSE-scores in YOD as compared to LOD around the time of diagnosis have been reported ${ }^{33,34}$. However, this difference may be explained by the higher proportion of YO-FTD-patients in these studies. A similar study, comparing only AD patients showed the YOD group was more cognitively impaired than the LOD group at time of diagnosis ${ }^{35}$, while another study assessing only FTD patients showed YO-FTD patients to be less cognitively impaired than LO-FTD patients ${ }^{36}$.

An additional explanation of the predictive value of younger age at onset may be that people are less inclined to consider dementia as a possible source of symptoms in young patients as has been suggested by other researchers ${ }^{37-39}$. YOD also has a vastly differential diagnosis: it includes not only atypical dementias and rare sporadic or hereditary diseases ${ }^{9,}{ }^{40}$, but also psychiatric conditions. Some studies have reported the relatively frequent occurrence of behavioural and personality changes as a presenting sign in patients with $\mathrm{YOD}^{8,12,13}$. Whereas behavioural problems in older patients seem to trigger the timely and adequate recognition of dementia by caregivers ${ }^{41}$, in younger people they may be more likely to be explained in psychosocial terms. Our previous qualitative report on the experiences of YOD caregivers in the period prior to diagnosis showed that most caregivers did not attribute symptoms to dementia and were likely to link behavioural changes to psychological causes ${ }^{13}$. In line with this notion, FTD- caregivers have been found to experience the failure to recognize the early stages of illness as dementia, as a major troublesome aspect ${ }^{42}$.

Our previous report also showed that initial non-dementia diagnoses in YOD cases are relatively common and several caregivers felt that the medical profession was not responsive when they sought help ${ }^{13}$. Another survey on YOD found that a higher number of diagnostic problems, such as misdiagnosis, was related to patient and caregiver's younger age ${ }^{14}$. Persons suffering from FTD are more likely to receive an initial diagnosis indicating that they have burnout or depression than patients with $\mathrm{AD}$, probably because FTD is characterised by behavioural changes in conjunction with a relatively preserved memory capacity ${ }^{12}$. These factors may therefore also contribute to a longer duration from symptom onset to diagnosis in YOD. In our study, the effect of age of onset on time to diagnosis was relatively small, with only $10 \%$ of the variance explained. We acknowledge that the model proposed does not nearly describe the interplay of all possible factors influencing time to diagnosis. Aspects such as denial among patients ${ }^{13}$, type of diagnostic service $^{43}$, patient residing in a rural versus an urban area ${ }^{44}$, contact rate with the primary care physician ${ }^{45}$, and primary care physician attitude towards the early 
detection of dementia are important ${ }^{46}$ but unfortunately have not been assessed in the current study. Furthermore, it could be that age does not explain a greater part of the variance because distinct factors related to older age can cause a diagnostic delay in this age group. For example, patients and their relatives as well as GPs may be more likely to put down memory problems to 'old age' in the elderly than in younger people, or attribute them to comorbidities or medication use ${ }^{47,48}$.

\section{Strengths and limitations}

The strength of the present study is that the factors related to time to diagnosis were investigated and compared in a relatively large sample of both YOD and LOD subjects, whereas previous studies presented qualitative data and/or data from smaller studies focusing on one age-group. Furthermore, different diagnostic groups were compared. Some potential limitations, however, also exist. Since dementia commonly has an insidious onset, it is by definition difficult to accurately time the onset ${ }^{49}$. Therefore we used the year of symptom onset of which estimation variation will average out over groups. Memory bias could be a factor in our study given that the moment when symptoms first appeared was assessed retrospectively. The recruitment strategy for the YOD patients differed from the strategy that was used in the MAASBED study, which may have also biased our results. While the LOD group from the MAASBED study was recruited both from a University Memory Clinic (MUMC) and from the Regional Institute for Community Mental Health Care (RIAGG) in Maastricht, the YOD patients were recruited through more diverse settings across the Netherlands including specialized day care centres in 115 cases. The interval between diagnosis and study-entry was larger in the YOD group, therefore recall bias could be more pronounced in this group. By contrast, memory problems in the LOD caregivers associated with their advanced age could have led to recall bias in this group. Also the fact that older people may be more likely to dismiss memory problems as 'just ageing' may be a confounding factor. In addition, the dementia diagnoses were not standardised, although regular criteria were used. Finally, the FTD and other-dementia subgroups were rather small and may not be representative of the intended populations. Especially in the LOD group these subsamples were small, because of the inherent differences between YOD and LOD, with more heterogeneous aetiologies and a higher prevalence of FTD at a younger age. This may have resulted in an overestimation of the effect of FTD on time to diagnosis. In addition, the outcomes of the other-dementia subgroup are by definition hard to compare between YOD and LOD, because this is not a homogeneous category. The results regarding time to diagnosis of these categories should therefore be interpreted with caution and replicated in larger homogeneous samples. However, a post-hoc analysis showed that including these categories did not unduly influence our results of the impact of age at onset and having a diagnosis of $\mathrm{VaD}$ on time to diagnosis. 


\section{Implications}

As yet, it is not clear which factors cause the extended time from symptom onset to diagnosis in YOD. Future studies should therefore assess the influence of factors such as denial among patients, misattribution of symptoms, type of diagnostic service and primary care physician attitude towards the early detection of dementia on time to diagnosis in YOD and LOD within large homogeneous diagnostic samples. In addition, our finding that FTD, independent of age at onset, results in a delay in diagnosis should be replicated in a larger sample of YOD as well as LOD patients. This would provide important issues to focus on when supporting patients and caregivers in the diagnostic trajectory as well as starting points to facilitate timelier diagnoses in both groups. The present study together with our previous qualitative report shows that the time from symptom onset to diagnosis is relatively long in YOD, that it is considered too long by many caregivers and that this delay may result in detrimental psychosocial consequences for YOD families ${ }^{13}$. In addition, it shows that not only in YO-FTD patients time to diagnosis is longer than in their older counterparts, but also in other YOD diagnostic categories. Hence, we advocate that efforts should be made to reach a more timely diagnosis in YOD patients. Therefore, our recommendations are aimed at promoting public awareness of YOD as well as increasing knowledge among the medical profession. In addition, the development and/or enhancement of specialised diagnostic centres for the diagnosis of YOD are important, because a structured approach based on all clinical features is required to diagnose $\mathrm{YOD}^{10}$. These centres may in turn increase awareness of this subgroup in primary care ${ }^{12}$. However, in promoting awareness of YOD we must be alert to the risk of false positive diagnoses, which may even lead to more harmful consequences. Previous research indicated that erroneously diagnosing psychiatric disease as dementia, is common in YOD ${ }^{50,51}$, therefore we emphasize the urgent need to maintain, or better still, improve the standards of diagnostic accuracy of YOD.

In conclusion, the present study underlines the need to raise special awareness of young-onset dementia, to facilitate a timely diagnosis, but also demonstrates that more research is necessary focussing on the mechanisms that cause an extended time between symptom onset and diagnosis. 


\section{References}

1. Salloway S, Correia S. Alzheimer disease: time to improve its diagnosis and treatment. Cleveland Clinic Journal of Medicine 2009;76:49-58.

2. Vernooij-Dassen MJFJ, Moniz-Cook ED, Woods RT, et al. Factors affecting timely recognition and diagnosis of dementia across Europe: From awareness to stigma. International Journal of Geriatric Psychiatry 2005;20:377-386.

3. Wilkinson D, Stave C, Keohane D, Vincenzino O. The role of general practitioners in the diagnosis and treatment of Alzheimer's disease: a multinational survey. The Journal of International Medical Research 2004;32:149-159.

4. Bamford C, Eccles M, Steen N, Robinson L. Can primary care record review facilitate earlier diagnosis of dementia? Family Practice 2007;24:108-116.

5. Holzer C, Warshaw G. Clues to early Alzheimer dementia in the outpatient setting. Archives of Family Medicine 2000;9:1066-1070.

6. Iliffe S, Wilcock J, Haworth D. Obstacles to Shared Care for Patients with Dementia: A qualitative study. Family Practice 2006;23:353-362.

7. Iliffe S, Manthorpe J. The hazards of early recognition of dementia: A risk assessment. Aging \& Mental Health 2004;8:99-105.

8. Mendez MF. The accurate diagnosis of early-onset dementia. International Journal of Psychiatry in Medicine 2006;36:401-412.

9. Ridha B, Josephs KA. Young-onset dementia: a practical approach to diagnosis. The neurologist 2006;12:2-13.

10. Rossor MN, Fox NC, Mummery CJ, Schott JM, Warren JD. The diagnosis of young-onset dementia. Lancet Neurology 2010;9:793-806.

11. Kelley BJ, Boeve BF, Josephs KA. Cognitive and noncognitive neurological features of young-onset dementia. Dementia and Geriatric Cognitive Disorders 2009; 27:564-571.

12. Rosness TA, Haugen PK, Passant U, Engedal K. Frontotemporal dementia-A clinically complex diagnosis. International Journal of Geriatric Psychiatry 2008;23: 837-842.

13. van Vliet D, de Vugt ME, Bakker C, et al. Caregivers' perspectives on the prediagnostic period in early onset dementia: a long and winding road. International Psychogeriatrics 2011:1-12.

14. Luscombe G, Brodaty H, Freeth S. Younger people with dementia: Diagnostic issues, effects on carers and use of services. International Journal of Geriatric Psychiatry 1998;13:323-330.

15. Tedstone Doherty D, Kartalova-O'Doherty Y. Gender and self-reported mental health problems: predictors of help seeking from a general practitioner. British Journal of Health Psychology 2010;15:213-228.

16. Tijhuis MA, Peters L, Foets M. An orientation toward help-seeking for emotional problems. Social Science \& Medicine 1990;31:989-995. 
17. Mackenzie CS, Gekoski WL, Knox VJ. Age, gender, and the underutilization of mental health services: The influence of help-seeking attitudes. Aging 83 Mental Health 2006;10:574-582.

18. Ramakers IHGB, Visser PJ, Bittermann AJN, Ponds RWHM, van Boxtel MPJ, Verhey FRJ. Characteristics of help-seeking behaviour in subjects with subjective memory complaints at a memory clinic: A case-control study. International Journal of Geriatric Psychiatry 2009;24:190-196.

19. Pentzek M, Wollny A, Wiese B, et al. Apart from nihilism and stigma: What influences general practitioners' accuracy in identifying incident dementia. The American Journal of Geriatric Psychiatry 2009;17:965-975.

20. van Vliet D, Bakker C, Koopmans RT, Vernooij-Dassen MJ, Verhey FR, de Vugt ME. Research protocol of the NeedYD-study (Needs in Young onset Dementia): a prospective cohort study on the needs and course of early onset dementia. BMC Geriatrics 2010;10:13.

21. American Psychiatric Association. Diagnostic and Statistical manual of mental Disorders- Text revision: DSM-IV-TR. American Psychiatric Association 2000.

22. CBO: Guideline diagnosis and pharmacological treatment of dementia. 2005. Available at: http://www.cbo.nl/Downloads/387/rldement2005.pdf. Accessed: March 3, 2012.

23. McKhann G, Drachman D, Folstein M, Katzman R, Price D, Stadlan EM. Clinical diagnosis of Alzheimer's disease: report of the NINCDS-ADRDA Work Group under the auspices of Department of Health and Human Services Task Force on Alzheimer's Disease. Neurology 1984;34:939-944.

24. McKeith IG. Consensus guidelines for the clinical and pathologic diagnosis of dementia with Lewy bodies (DLB): report of the Consortium on DLB International Workshop. Journal of Alzheimer's Disease 2006;9:417-423.

25. Neary D, Snowden JS, Gustafson L, et al. Frontotemporal lobar degeneration: a consensus on clinical diagnostic criteria. Neurology 1998;51:1546-1554.

26. Mesulam MM, Grossman M, Hillis A, Kertesz A, Weintraub S. The core and halo of primary progressive aphasia and semantic dementia. Annals of Neurology 2003;54 Suppl 5:S11-14.

27. Erkinjuntti T. Clinical criteria for vascular dementia: The NINDS-AIREN criteria. Dementia 1994;5:189-192.

28. Aalten P, van Valen E, de Vugt ME, Lousberg R, Jolles J, Verhey FRJ. Awareness and behavioral problems in dementia patients: A prospective study. International Psychogeriatrics 2006;18:3-17.

29. Reisberg B, Ferris SH, de Leon MJ, Crook T. The Global Deterioration Scale for assessment of primary degenerative dementia. American Journal of Psychiatry 1982;139:1136-1139.

30. Passant U, Elfgren C, Englund E, Gustafson L. Psychiatric Symptoms and Their Psychosocial Consequences in Frontotemporal Dementia. Alzheimer Disease and Associated Disorders 2005;19:S15-S18. 
31. Reilly J, Rodriguez AD, Lamy M, Neils-Strunjas J. Cognition, language, and clinical pathological features of non-Alzheimer's dementias: an overview. Journal of Communication Disorders 2010;43:438-452.

32. Braak H, Braak E. Frequency of stages of Alzheimer-related lesions in different age categories. Neurobiology of Aging 1997;18:351-357.

33. Papageorgiou SG, Kontaxis T, Bonakis A, Kalfakis N, Vassilopoulos D. Frequency and causes of early-onset dementia in a tertiary referral center in Athens. Alzheimer Disease and Associated Disorders 2009;23:347-351.

34. McMurtray A, Clark DG, Christine D, Mendez MF. Early-Onset Dementia: Frequency and Causes Compared to Late-Onset Dementia. Dementia and Geriatric Cognitive Disorders 2006;21:59-64.

35. Picard C, Pasquier F, Martinaud O, Hannequin D, Godefroy O. Early onset dementia: Characteristics in a large cohort from academic memory clinics. Alzheimer Disease and Associated Disorders 2011;25:203-205.

36. Shinagawa S, Toyota Y, Ishikawa $\mathrm{T}$, et al. Cognitive function and psychiatric symptoms in early- and late-onset frontotemporal dementia. Dementia and Geriatric Cognitive Disorders 2008;25:439-444.

37. Werner P, Stein-Shvachman I, Korczyn AD. Early onset dementia: clinical and social aspects. International Psychogeriatrics 2009:1-6.

38. Harris PB, Keady J. Living with early onset dementia: exploring the experience and developing evidence-based guidelines for practice. Alzheimer's Care Quarterly 2004;5:111-122.

39. Williams T, Cameron I, Deardon T. From pillar to post-a study of younger people with dementia. Psychiatric Bulletin 2001;25:384-387.

40. Sampson EL, Warren JD, Rossor MN. Young onset dementia. Postgraduate Medical Journal 2004;80:125-139.

41. Eustace A, Bruce I, Coen R, et al. Behavioural disturbance triggers recognition of dementia by family informants. International Journal of Geriatric Psychiatry 2007;22:574-579.

42. Chow TW, Pio FJ, Rockwood K. An international needs assessment of caregivers for frontotemporal dementia. The Canadian Journal of Neurological Sciences 2011;38:753-757.

43. Cordery R, Harvey R, Frost C, Rossor M. National survey to assess current practices in the diagnosis and management of young people with dementia. International Journal of Geriatric Psychiatry 2002;17:124-127.

44. Koller D, Eisele M, Kaduszkiewicz H, et al. Ambulatory health services utilization in patients with dementia - Is there an urban-rural difference? International Journal of Health Geographics 2010;9:59.

45. Eefsting JA, Boersma F, Van Den Brink W, Van Tilburg W. Differences in prevalence of dementia based on community survey and general practitioner recognition. Psychological Medicine: A Journal of Research in Psychiatry and the Allied Sciences 1996;26:1223-1230. 
46. Hansen EC, Hughes C, Routley G, Robinson AL. General practitioners' experiences and understandings of diagnosing dementia: factors impacting on early diagnosis. Social Science \& Medicine 2008;67:1776-1783.

47. Cahill S, Clark M, Walsh C, O'Connell H, Lawlor B. Dementia in primary care: the first survey of Irish general practitioners. International Journal of Geriatric Psychiatry 2006;21:319-324.

48. Leung KK, Finlay J, Silvius JL, et al. Pathways to diagnosis: Exploring the experiences of problem recognition and obtaining a dementia diagnosis among Anglo-Canadians. Health \& Social Care in the Community 2011;19:372-381.

49. Ratnavalli E, Brayne C, Dawson K, Hodges JR. The prevalence of frontotemporal dementia. Neurology 2002;58:1615-1621.

50. Ron MA, Toone BK, Garralda ME, Lishman WA. Diagnostic accuracy in presenile dementia. The British Journal of Psychiatry 1979;134:161-168.

51. Marsden CD, Harrison MJ. Outcome of investigation of patients with presenile dementia. British Medical Journal 1972;2:249-252. 



\section{Chapter 5}

\section{Caregivers' perspectives on the pre-diagnostic period in young-onset dementia}

Based on: van Vliet D, de Vugt ME, Bakker C, Koopmans RTCM, Pijnenburg YAL, Vernooij-Dassen MJFJ, Verhey FRJ (2011) Caregivers' perspectives on the pre-diagnostic period in early-onset dementia: a long and winding road. International Psychogeriatrics. 


\begin{abstract}
Background Recognizing and diagnosing young-onset dementia (YOD) can be complex and often takes longer than for late-onset dementia. The objectives of this study are to investigate the barriers to diagnosis and to develop a typology of the diagnosis pathway for YOD caregivers.
\end{abstract}

Methods Semi-structured interviews with 92 YOD caregivers were analyzed using constant comparative analysis and grounded theory. A conceptual model was formed based on 21 interviews and tested in 29 additional transcripts. The identified categories were quantified in the whole sample.

Results Seven themes emerged: (1) changes in the family member, (2) disrupted family life, (3) misattribution, (4) denial and refusal to seek advice (5) lack of confirmation from social context, (6) non-responsiveness of a general practitioner (GP) and (7) misdiagnosis. Cognitive and behavioral changes in the person with YOD were common and difficult to understand for caregivers. Marital difficulties, problems with children and work/financial issues were important topics. Confirmation of family members and being aware of problems at work were important for caregivers to notice deficits and/or seek help. Other main issues were a patient's refusal to seek help resulting from denial and inadequate help resulting from misdiagnosis.

Conclusion YOD caregivers experience a long and difficult period before diagnosis. We hypothesize that denial, refusal to seek help, misattribution of symptoms, lack of confirmation from the social context, professionals' inadequate help and faulty diagnoses prolong the time before diagnosis. These findings underline the need for faster and more adequate help from health-care professionals and provide issues to focus on when supporting caregivers of people with YOD. 


\subsection{Introduction}

The detection and diagnosis of dementia that begins before the age of 65 is often a challenge for clinicians and takes longer than in the elderly ${ }^{1}$. Factors causing a delay include the lower prevalence and the broader differential diagnosis compared with late- onset dementia (LOD) ${ }^{2-4}$. In addition, the clinical presentation of many types of young-onset dementia (YOD) is marked by neuropsychiatric symptoms instead of cognitive disturbances ${ }^{3,5}$. Therefore, it is common for there to be a psychiatric diagnosis preceding the dementia diagnosis in $\mathrm{YOD}^{2,3,6}$.

As a consequence, people with YOD and their caregivers are at risk of undergoing a large number of referrals and may feel like they are being 'passed from pillar to post' in the period prior to diagnosis ${ }^{7}$. It has also been found that being younger and appearing to be physically fit and healthy makes it more difficult for some people with YOD to receive a correct and early diagnosis ${ }^{8}$. The prolonged time to reach an accurate diagnosis often leads to frustration about the medical profession in caregivers ${ }^{8}$. Other problems that caregivers may experience during this period are: inadequate help, lack of professionals' knowledge and feeling responsible for finding available help themselves. These issues are distressing to people suffering from YOD and their caregivers ${ }^{6-9}$.

It is not only diagnostic uncertainty or misdiagnosis that can cause a delay in diagnosing dementia. Younger people and their relatives are also less likely to even consider the possibility of dementia, leading to a delay in seeking help ${ }^{10}$. It has been reported that caregivers of individuals with YOD thought the initial symptoms were temporary exacerbations of character or that they were due to stress or depression. They only contacted their GP after a significant life event ${ }^{7}$. In a study of young-onset Alzheimer's disease and vascular dementia, Sperlinger and Furst (1994) reported that work-related problems were the primary initial symptoms, whereas Newens et al. (1994) reported memory deficits, disorientation and lack of energy in another study on young- onset Alzheimer's disease ${ }^{11}$.

None of the studies reported the main concerns of YOD caregivers within the period from symptom onset to diagnosis. It is important to explore the experiences of caregivers within this period, because it could give further insight into the factors and dynamics that contribute to the prolonged time before diagnosis. Such studies have been conducted in dementia ${ }^{12-14}$, but to the best of our knowledge, none of them have focused on YOD. Therefore, the goal of this paper is to investigate the barriers to obtaining a dementia diagnosis for caregivers of people with YOD and to develop a typology of the diagnosis pathway from the perspectives of these caregivers. 


\subsection{Methods}

\section{Participants}

The present study is part of an ongoing prospective cohort study, the NeedYDstudy (Needs in Young-onset Dementia). More details have been described elsewhere ${ }^{15}$. In short, the NeedYD-study is a prospective cohort study in which people with YOD and their caregivers $(\mathrm{N}=215)$ are followed up for two years and undertake measurements every six months, including semi-structured and structured interviews, questionnaires and cognitive tests. The main objectives are to investigate the (un)met needs of individuals with YOD and their family members and to investigate the course of neuropsychiatric symptoms and their possible risk factors. An additional aim is to explore the experiences and feelings of people with YOD and their caregivers during the diagnostic period. The NeedYD-study consists of two community-dwelling cohorts: one in the diagnostic phase (i.e., those not yet receiving specialized day care) and one receiving specialized day care. For the present study, the data from the cohort of people with YOD and their caregivers in the diagnostic phase were selected $(\mathrm{N}=100)$ because they were expected to be included shortly after receiving the diagnosis. The caregivers were recruited through the memory clinics of the Maastricht University Medical Center+ (MUMC+) and the VU University Medical Center (VUMC) in the Netherlands, regional hospitals and regional mental health services in the southern and central parts of the Netherlands. In addition, some caregivers applied individually to participate.

\section{Data collection}

Written informed consent was obtained before participation in the study. A semistructured interview was administered to the caregivers. The interviews were administered and audio taped at the caregivers' homes. Each section started with an open question followed by more specific sub-questions. When needed, the interviewer asked caregivers to clarify or to expand their answers. Caregivers were also encouraged to explain their feelings or thoughts about issues they brought forward. For the present study, we selected the parts of the interview that addressed topics concerning the period prior to diagnosis (Box 1). Apart from this interview, caregivers were asked in which year the first symptoms occurred. In addition, the patients' medical files were consulted to obtain the year of diagnosis and medical history. Researchers had internal access to 55 medical records from the VUMC and MUMC+. The remaining medical information from external hospitals was requested by mail. Furthermore, the Mini-Mental State Examination (MMSE) ${ }^{16}$ was administered to the patients. 
Box 1. Questions asked at the interview with YOD caregivers concerning the period prior to diagnosis

1. When did you first notice something was wrong with your family member?

a) What did you notice?

2. Can you describe the period prior to diagnosis?

a) What did you think was wrong?

b) Which problems did you experience during this period?

c) Which problems did the children encounter?

d) When did you seek help?

e) Where did you seek help?

f) Can you tell me how you were helped from that point onwards?

\section{Analysis}

The interviews were completely transcribed verbatim. The transcripts were analyzed using ATLAS.ti software ${ }^{17}$. We used constant comparative analysis and applied a grounded theory approach ${ }^{18,19}$ to assess four steps in the diagnostic process: (1) problems experienced, (2) suspicion and interpretation, (3) seeking help and (4) the referral trajectory. A three-step procedure was used in the analysis.

First, two researchers ( $\mathrm{DvV}$ and $\mathrm{MdV}$ ) independently analyzed a subset of interviews in consecutive order until, after 21 transcripts, saturation of data occurred (i.e., no new information was obtained). During this analysis, the researchers coded the transcripts thoroughly and constantly compared the content of pieces of transcript with the codes that were established. After establishing the codes, they were grouped into categories and these categories were then grouped into major themes. Based on this first analysis, a framework for a theory was established.

Second, the theory was tested in an additional 29 interviews by constant comparison by one researcher $(\mathrm{DvV})$. The theory was then verified by another researcher $(\mathrm{AD})$, who read all the pieces of transcripts per category. She looked at the interrelations between codes and categories, in particular, and identified the most important issues per category. If there were different views on the results of the analysis, these were discussed and incorporated in the results. This step is necessary because in this type of study, the existence of inter-coder differences should be recognized ${ }^{20}$.

Third, the categories that resulted from the first two steps were identified and quantified in the whole sample by one researcher (DvV). Another researcher analyzed a random sample of 20 interviews in the same way (AD).

In addition to the quantification of the identified categories, the codes making 
up the categories concerning the initial symptoms and the referral trajectory were also quantified. Results regarding the referral trajectory were verified by assessing the patients' medical histories.

\subsection{Results}

\section{The sample}

Data from eight caregivers were missing. Table 5.1 presents the characteristics of the included group $(\mathrm{N}=92)$ of people with YOD and their caregivers.

The diagnoses of the people with YOD were: Alzheimer's disease (AD) (68\%), frontotemporal lobar degeneration (FTLD) (17\%), vascular dementia or mixed dementia (VaD/AD) (10\%) or another cause (5\%). In the group of people with FTLD, eight suffered from frontotemporal dementia and seven from primary progressive aphasia. The mean MMSE score of the whole group (minus the missing / non-reliable scores) was $20.1(\mathrm{SD}=6.8 ; \mathrm{N}=74)$. Time from symptom onset to diagnosis was calculated to compare the group for which we did not have interviews $(\mathrm{N}=8)$ with the group for which we had interviews $(\mathrm{N}=92)$. In the group with interviews, it took an average of 4.6 years $(\mathrm{SD}=3.2)$ to obtain a diagnosis and in the missing group 4.1 years $(\mathrm{SD}=3.8)$. The group without interviews $(\mathrm{N}=8)$ had a mean MMSE score of $21.0(\mathrm{SD}=5.8)$ at baseline. The group with interviews and the group without did not differ in terms of MMSE score $(U=386.5, z=-0.149$, $\mathrm{p}=.882)$ or time to diagnosis $(U=309.0, z=-0.756, \mathrm{p}=.449)$.

\section{Findings}

The analysis of the four steps in the diagnostic process resulted in the identification of the following themes: (1) changes in the family member, (2) disrupted family life, (3) misattribution of symptoms, (4) denial, (5) lack of confirmation from social context, (6) lack of responsiveness of the GP and (7) faulty diagnosis.

\section{Changes in the family member}

This theme relates to the experience of the caregiver noticing changes in the person who is eventually diagnosed with dementia, ranging from feelings of uneasiness to a clear suspicion that something is wrong. Apart from cognitive changes, caregivers also frequently reported behavioral changes.

Most caregivers did not recognize the changing behavior of their spouse or family member as deviant at the time it first occurred. Only in retrospect, once the diagnosis of dementia was established, were they able to understand the initial subtle changes in cognition, behavior and/or daily functioning. Some caregivers mentioned more pronounced symptoms as first signs, such as delusional behavior.

One caregiver described the first symptoms displayed by his 61 -year-old wife with AD: "I did not really give it a name at first, but I noticed that she got less 
Table 5.1: Characteristics of the participants $(\mathrm{N}=92)$

\begin{tabular}{lcc}
\hline & $\begin{array}{c}\text { YOD } \\
\text { patients }\end{array}$ & $\begin{array}{c}\text { YOD } \\
\text { caregivers }\end{array}$ \\
\hline Mean duration from symptom onset to diagnosis (year) & $4.6 \pm 3.2(0-18)$ & \\
$\pm \mathrm{SD}$ (range) & & \\
Mean duration from diagnosis to baseline assessment & $1.8 \pm 2.0(0-11)$ & \\
(year) $\pm \mathrm{SD}$ (range) & $45 / 47$ & $44 / 48$ \\
Sex male/female (N) & $60.6 \pm 4.8$ & $59.3 \pm 8.7$ \\
Mean age \pm SD (range) & $(43.4-68.8)$ & $(25.2-78.3)$ \\
& & $88|1| 3$ \\
Caregiver is partner| sibling| child (N) & 7 & 48 \\
Working at baseline assessment (N) & 84 & \\
Caregiver and patient live together (N) & $23($ of 17 patients) \\
Children living at home at baseline assessment (N) & $54.3 \pm 5.8$ & $53.0 \pm 9.3$ \\
Mean age at disease onset \pm SD (range) & $(38.4-64.8)$ & $(16.2-76.3)$ \\
& & \\
Childrens' age at disease onset (N) & 1 & \\
Unborn & 7 & \\
0-10 years & 30 & \\
11-20 years & 77 & \\
$21-30$ years & 48 & \\
Above 30 years & 28 & \\
Stopped working in period prior to diagnosis, because & & \\
of dementia (N) & & \\
\hline
\end{tabular}

interested in the newspaper or television programs she used to watch. I noticed that there was only interest for a music or fun television program, but for a conversation there was no interest at all. That was nonsense in her opinion."

Behavioral changes were quite common as a first symptom (43\%). Apathy and lack of social reciprocity were highly prevalent. Forty percent of caregivers mentioned only cognitive problems. These symptoms were mentioned in combination with behavioral symptoms in $17 \%$ of the cases and with functional impairment in $14 \%$. Eighteen percent of the caregivers reported only behavioral disturbances as the first symptoms. In some cases, the changes only became noticeable after a patient lost his/her job and was at home more often.

As time progressed, cognitive and behavioral changes and a decline in activities of daily living were reported to become more profound during the period prior to diagnosis, with accumulating problems within the family and incomprehension of the caregiver. Within this period most caregivers (85\%) noticed cognitive changes, especially memory problems.

A caregiver described the early memory problems of her 60-year-old husband with AD: "My husband became forgetful, forgetting appointments or where he put his keys. He did not just forget things, but he wasn't even capable of imagining 
places where he could have left his keys. Normally when you forget something, you remember again when someone reminds you. But he did not have that; it just didn't come back."

Personality changes and problem behaviors were mentioned by $57 \%$ of the caregivers. Loss of interest/apathy, loss of social reciprocity and irritability/agitation were the most prevalent.

One caregiver mentioned the lack of responsiveness to her needs by her 60year-old husband with dementia NOS (not otherwise specified): "The behavior of my husband changed. He just did not respond when I needed help or when I needed an arm around my shoulder."

In some cases, caregivers blamed their family member for their apathy or for being self-centered and reacted strongly.

This was expressed by a caregiver about her 62-year-old husband with $\mathrm{VaD}$ in the following way: "His behavior changed; he lost interest in us. Yes, I worried, but I was also angry with him."

\section{Disrupted family life}

This theme expresses the problems within the family life of YOD patients. The changes in the patient may disrupt the basic daily routines in family life and can cause tension.

Family/marital conflict Problems within the relationship were mentioned by $33 \%$ of the caregivers. Most of these caregivers described the period prior to diagnosis as tense or full of anger, misunderstanding and conflicts between them and their spouse. Memory problems led to tension and quarrels between the caregiver and their spouse. Caregivers were confronted by their family member denying something had happened, sometimes blaming the caregiver for making things up.

One caregiver expressed how his 59-years-old wife with $\mathrm{VaD} / \mathrm{AD}$ reacted as a result of her memory problems: "She forgot a lot of things and blamed me. If I did not remember something she said I had not listened, according to her. If she did not remember something I said, I had not told her."

Another caregiver described how she reacted to the memory problems displayed by her 62-year-old husband with $\mathrm{AD}$ : "The memory problems caused friction and impatience. From my side, of course, especially in the beginning there was a lot of impatience. I frequently reacted with: 'I already told you, we just talked about that."”

The personality changes and behavioral problems were most often mentioned with regard to major conflicts and serious disruptions to the marital relationship. Five caregivers even reported being on the verge of leaving their spouse. They emphasized the importance of knowing the diagnosis.

One caregiver stated she was on the verge of leaving her 67-year-old husband with AD: "In that period he constantly went wandering on the streets at night. 
After that there was a period he was stalking me. At a certain point I thought, 'this is it, now I am going to leave him.', but my children prevented me from doing that."

Twenty-eight percent of the caregivers with children $(\mathrm{N}=74)$ reported that their children suffered from difficulties in the period prior to diagnosis. The children, especially those who were living at home at that time or were in their adolescence, came into conflict with their ill parent, because of irritation and misunderstandings.

Two caregivers described what it was like in the period prior to diagnosis for them and their children:

"It just was difficult in the household. Potatoes were boiled, but no water was in the pan. Dinner was ready, but the meat was still in the freezer and so on. Such things happened. You don't want to sit at the table with teenagers and dinner is not ready. That always caused tension. In that sense the diagnosis was a pleasure."

"It was a terrible period in which you would rather stay away from home because of the tension my husband brought. He was very nasty to the children. And a child cannot think about that rationally. A child of 13 does not think like an adult."

Other problems caregivers mentioned were, for example, children noticing tension between their parents or receiving less attention from their parents.

Work and financial issues Of the patients who still worked at the time their first symptoms occurred $(\mathrm{N}=65), 52 \%$ of the caregivers reported problems with regard to work or financial issues. Caregivers mentioned several difficulties, such as patients being less productive in their jobs, being unable to manage their previous routine tasks or having conflicts at work. Caregivers had to deal with the emotional impact on their family member and support them by, for example, talking to their employers. For caregivers, it was hard to understand why their family member encountered these problems.

A caregiver described the problems at work of his 58-year-old wife with AD: "She experienced a lot of grief; she did not understand anything about all the accusations she received at work. They accused her of neglecting things at work and I had to go out of my way to comfort her."

Another problem that was frequently mentioned concerned the patients not informing their caregivers about the problems they experienced at work. Some caregivers reported that patients lost their jobs, sometimes leading to a decrease in income. At this stage, patients were often obliged to apply for a new job, although they were mostly not capable of doing that. One patient did find a new job, but was dismissed again.

This caregiver mentioned the importance of knowing the diagnosis of her 61year-old husband with frontotemporal dementia: "He got fired constantly, one dismissal after another. I was happy they found out what was wrong with my husband. I only had a small income and then he got declared disabled and we got money from the health insurance. That made a difference." 


\section{Misattribution of symptoms}

This theme describes how the occurring symptoms were interpreted by caregivers and which factors were involved in the decision-making process leading them to seek help.

The majority of caregivers were not aware that their family member suffered from dementia when initial symptoms occurred. Some caregivers attributed symptoms to aging.

For instance a caregiver stated about his 68 year old wife suffering from $\mathrm{AD}$ that he thought her memory problems were normal: "To me her forgetfulness was not abnormal. People tend to get more forgetful when they get older. I also noted that she was not able to calculate the scores anymore when we played cards and she did not like to do that anymore. I noticed that, but did not think it was important."

Some caregivers did not notice any changes for a while and looking back on that period they tried to explain why they did not notice the changing behavior of their spouse:

One caregiver related it to characteristics that his 61-year-old wife with $\mathrm{AD}$ had always had: "After my sister-in-law pointed out my wife suffered from memory problems, I noticed that she forgot more appointments than she used to. My wife always was a forgetful person, so these problems were not obvious to me before."

Another caregiver explained not noticing changes in his 64-year-old wife with dementia NOS because they spent so much time together: "Close family knew beforehand something was wrong. I mean, my two daughters are pharmacists with a PhD degree, so they know the story. They came home once in a while and they saw things much clearer. I mean, I saw my wife every day, so I didn't see the nuances that clear any more. My daughters said, listen dad; 'when I come in and walk through the house and look in the closet I see what happens here."

There was also a group of caregivers who did not have any idea of what could be wrong.

For example one caregiver described noticing that his 64-year-old wife with $\mathrm{AD}$ changed, but did not know why this happened: "If your wife always has been active, but she stops doing that all of a sudden, you think: what is going on? However, I never knew what to make of it; I was never able to label this behavior. I only thought it was very annoying."

The largest proportion of caregivers thought the cause of the changes in their family member was psychological - e.g. burnout, depression or stress. The main reasons for this were problems at work or losing their jobs. Most of the caregivers attributing symptoms to psychological causes mentioned behavioral problems next to cognitive changes as the first sign of the disease. If potentially influential events occurred, these were also easily seen as possible causes of stress and changes in behavior.

A caregiver mentioned several reasons that would explain the changes in her 60-year-old husband with AD: "When he had to stay home, because of a burnout, 
I first noticed something was different. At that time I had recovered from cancer twice, so everything came all at once. I noticed he behaved strangely, but I thought this was because he could not take it anymore and his work was too burdensome for him."

Sometimes the reason was not found in concurrent events, but rather in the past.

For instance, a caregiver thought the changes could be due to the difficult childhood of his 64-year-old wife with AD: "I noticed a certain strain between us and the input in the relationship was not equal any more. But after several years of marriage you think you can get through it. I thought: 'this is a dark period, she suffered from a difficult childhood and things will work out eventually'. But everything just got worse."

There were also caregivers who thought of a neurological cause other than dementia, such as a brain tumor or vascular problems. Caregivers who mentioned these concerns either had a spouse with a family history of vascular disease running in the family or their family member suffered from physical complaints, such as headaches, hyperventilation or sleep apnea.

Eventually, because of worsening symptoms, some caregivers did suspect the cause could be dementia. Those caregivers mostly recognized symptoms because they were familiar with this condition in their own family. A small minority of caregivers did suspect dementia in the initial stage of the disease. In this group cognitive changes were commonly mentioned as presenting sign in the patient without behavioral changes.

\section{Seeking help}

Generally, it took a while before caregivers thought something was sufficiently wrong that they decided to consult a GP. In nearly every case, the caregiver rather than the person with dementia was the one to seek help. Patient-related factors that contributed to the feeling that something was wrong and help was necessary were the development of more pronounced memory complaints and the occurrence of a significant limitation in functioning, such as getting completely lost or losing the ability to cook.

An important turning point mentioned was the return from holidays. A break from the normal routines made caregivers more aware of the limitations of their family member. While on holidays the limitations were attributed to the change of environment, after returning home the changes were even more striking.

Further, the social context played an important role for caregivers in triggering the idea something was wrong or confirming their worries. Confirmation of others helped them in the decision to seek help. Family members, including children often pointed out the changes in behavior to caregivers and sometimes were able to convince the caregiver that they should consult a GP. Also, the interference of an 'outsider' (e.g. child, district nurse) sometimes helped to convince the patients themselves to find professional help. 
A caregiver pointed out that it was not until one of his children convinced him it was necessary, that he sought help for his 59-year-old wife with AD: "I first thought my wife was too busy at work, but later on one of my children said to me, 'let's go ask the GP.' That set things in motion."

Although family members commonly played an important role in recognizing symptoms, sometimes they were not aware of these symptoms, because patients were able to cover up their deficits to the outside world.

A caregiver of a 59-year-old male with primary progressive aphasia underlined the need to be understood by her family and friends: "That period was very tiring for me because I could not convince anyone that something was wrong. My husband, a giant of a man, knew very well how to manage in specific situations, so nobody noticed anything. He could really fool people."

A lack of understanding from family or friends, including children, made the period prior to diagnosis more difficult for caregivers and made it harder to seek medical advice.

\section{Hiding problems and denial}

A major barrier for caregivers to realize something was wrong and to seek help was the fact that patients were denying and hiding their problems.

Possible denial (33\%) showed itself in several different behaviors, namely: denying mistakes, denying something was wrong, trivializing difficulties, covering up cognitive deficits and hiding problems experienced, such as conflicts at work.

For example, one caregiver described how her 63-year-old husband with AD denied something was wrong with him and hid things from her: "I noticed his functioning declined, but he denied it. Finally he went to the GP, alone, because I was not allowed to go with him. When he came back he told me the GP said nothing was wrong. However, it appeared that my husband had received a referral letter, but he just ignored it."

Looking back, the first impairments in daily living usually started at work. However, often caregivers did not get any signals about these problems by their spouses nor were they informed by the employer. They commonly only became aware of the severity of problems when their spouse lost his/her job or, in a few cases, when caregivers contacted their spouses' employer. Awareness of these workrelated problems was an important factor contributing to the feeling something was wrong and help was necessary.

One caregiver described how her 47-year-old husband with frontotemporal dementia did not tell her about his difficulties at work: "When he got fired I heard afterwards what had happened. He appeared to have had weekly appointments with his superior and received a report every week. He never brought those reports home, but left them in his locker at work. And they stayed there, so I didn't know anything about his problems." 
Other caregivers had to rely on the accounts of their spouses, usually not representing an accurate picture of reality, because they were not able to reflect on their own functioning.

Once caregivers had decided to seek help, a major problem was resistance or refusal of their family member to consult a GP, because their family member did not think there was anything wrong.

A caregiver described how the refusal of his 61-year-old wife suffering from $\mathrm{AD}$ resulted in a delayed diagnosis, although he had already consulted the GP: "I mentioned it to the GP. He said 'if you come together, I can help you, but if she refuses to see me, there is nothing I can do for you'. So it took several years before I convinced my wife to go to the GP. She refused and said it wasn't true what I said. That was the problem."

\section{Non-responsiveness, misdiagnosis and inadequate help}

This theme focuses on the period after the patient and caregiver have decided to seek help. We found that YOD caregivers and patients encounter a considerable number of problems with professional health care.

A number of issues were brought forward by the caregivers about the referral trajectory. They felt that their GP did not take them seriously.

For instance, a caregiver described the non-responsiveness of the GP of her 65-year-old husband with AD: "I went to the GP several times, but he just did not respond, and I suffered from the consequences. He thought it was a depression and referred my husband to the regional mental health service. At a certain point I got frightened because of the memory disturbances, so I went to the GP again. I told him: 'I want you to do something, because I can no longer keep it up. You don't know what is happening and pass me from pillar to post. I demand a medical examination'."

Of the patients and their caregivers for whom a GP was the first clinician they consulted $(\mathrm{N}=79), 22 \%$ of the caregivers stated that their GP neglected to refer them after their first consultation. In the case of the other patients the situation was different, because they were under regular control for somatic conditions and got referred to a memory clinic by their specialists, or the caregiver asked the advice of an acquainted specialist directly, which could have influenced the decision of the GP to refer.

Furthermore, one of the main issues was the feeling of being passed 'from pillar to post'. Thirty-one caregivers $(34 \%)$ reported that their family member received an erroneous diagnosis prior to the dementia diagnosis either by their GP or a specialist. When patients received a psychological diagnosis they were sent home or were referred and received counseling for the established psychological conditions.

Overall, the referral trajectory was considered too long and some caregivers stated that they had to fight to get attention or a medical examination. Fifteen 
Table 5.2: Types of earlier diagnoses $(\mathrm{N}=92)$.

\begin{tabular}{lcc}
\hline Diagnosis & N & \% \\
\hline Based on interviews & & \\
Psychological & 12 & 13 \\
$\quad$ Burnout/depression & 11 & 12 \\
$\quad$ Other psychological (psychological NOS, & \\
$\quad$ marital problems, PTSD, anxiety) & \\
Somatic & 2 & 2 \\
$\quad$ Other neurological (infarctions) & 1 & 1 \\
$\quad$ COPD & 1 & 1 \\
$\quad$ Due to medication & 4 & 4 \\
Nothing wrong/No dementia diagnosis & & \\
Additional based on medical records & 9 & 10 \\
$\quad$ Burnout/depression * & 1 & 1 \\
$\quad$ Psychological NOS & 41 & 45 \\
Total & & \\
\hline * In two cases medical history showed chronic conditions. COPD, chronic obstructive pulmonary \\
disease; PTSD, post traumatic stress disorder; NOS, not otherwise specified.
\end{tabular}

percent of the caregivers explicitly reported that they felt relief after the dementia diagnosis was established.

A 26-year-old caregiver felt she and her mother, a 58-year-old with AD, did not get proper help before the dementia diagnosis was established: "The neurologist concluded my mother suffered from post-traumatic stress disorder, so he advised us not to worry and not to pay attention to the memory problems. My sister and I managed to do that until we began to worry again. Finally she got diagnosed with Alzheimer's disease. As soon as this word 'Alzheimer's' appeared, which of course is terrible to hear, we received help. It is almost a magical word, although it has a terrible meaning. Medical doctors take us serious for the first time. Thus, all the people who are still out there without a proper diagnosis don't get any help."

\section{Verification of other diagnoses based on medical history}

A total of 41 cases (45\%) were found to have received a diagnosis other than dementia, prior to their dementia diagnosis. These diagnoses are all listed in Table 5.2.

Twenty-six of the reported diagnoses in the interviews were consistent with the medical records, whereas fifteen cases of misdiagnosis were inconsistent in the interview and the medical record. Five diagnoses were mentioned by caregivers, but not found in the medical files. According to the caregivers these diagnoses were established by GPs. It is often the case that not all information about GP consultations is documented in hospital medical records, which can explain the fact that five diagnoses were not found. Ten diagnoses were found in the medical 


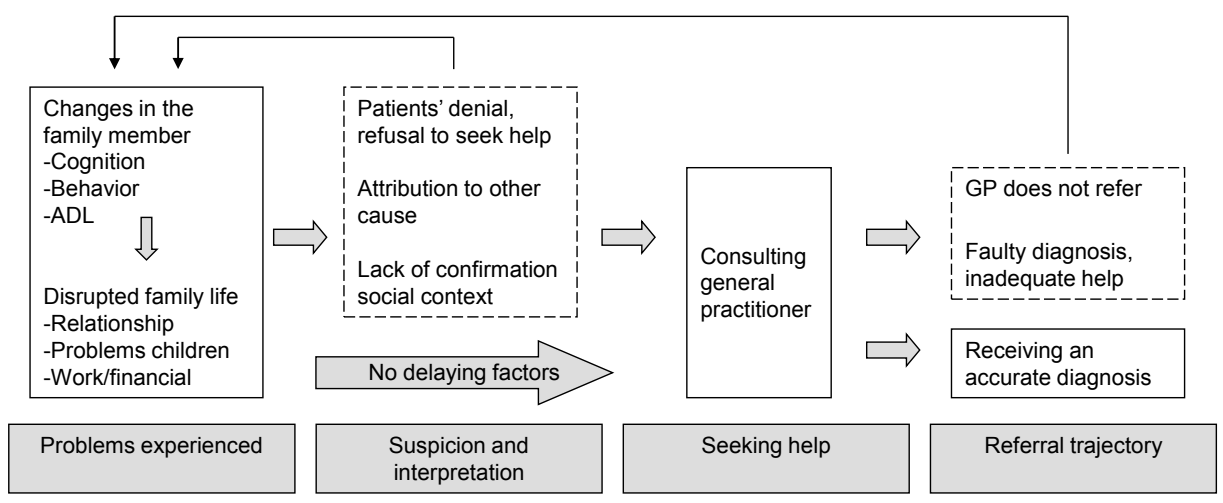

Figure 5.1: Graphic summary of results. Different possible trajectories from symptom onset to diagnosis emerging from the qualitative analysis of interviews with YOD caregivers. The dashed boxes represent possible factors prolonging the period before diagnosis.

records, but not mentioned by caregivers. Suspicions leading to wrong referrals were also taken into account; for example, a neurologist referred a patient to the regional mental health service because of suspected depression. Psychological diagnoses were the most common (36\%). From the medical records it appeared that in $15 \%$ of these cases, the diagnoses were maintained when the dementia diagnosis was established, indicating comorbidity. In one case, the diagnosis "mild cognitive impairment (MCI), no dementia" was established. The caregiver did not agree and obtained the dementia diagnosis through a second opinion. Furthermore, in three patients there were suspicions of autism in addition to another established psychological condition.

\section{Integration of themes}

The themes and categories that emerged from the qualitative analysis are integrated and presented in Figure 5.1. The different possible trajectories that YOD patients and caregivers follow are presented. In the model, there is a specific focus on which factors could be involved in the delay in receiving a diagnosis (boxes with dashed lines). The process starts with changes in the person with YOD, leading to the decision to seek help, which is often preceded by problems within the family. However, when the individual with YOD denies and refuses to seek medical advice, when symptoms are not accurately attributed or when caregivers do not receive any confirmation of their suspicions from others, the decision to seek help may be postponed. Meanwhile, symptoms may worsen and possible disruption to family life worsens/remains until people actively seek help. Similarly, if a medical doctor is consulted and inadequate help is offered (e.g. not being referred, erro- 
neous diagnosis), the unstable situation continues. Accurate diagnosis can break this vicious cycle. It should be noted that there were also people who did not experience these problems, as is indicated in the figure.

\subsection{Discussion}

The goal of this study was to investigate the barriers to obtaining a dementia diagnosis for caregivers of people with YOD and to develop a typology of the diagnosis pathway for these caregivers, by investigating four steps in the diagnostic process: (1) problems experienced, (2) suspicion and interpretation, (3) seeking help and (4) the referral trajectory.

We theorize that the following themes play a role in the delay in diagnosing YOD: (1) "misattribution of symptoms by caregivers", (2) "denial of the individual with YOD and the refusal to seek medical advice", (3) "lack of confirmation from the social context", (4) "lack of responsiveness of GP" and (5) "misdiagnosis of people with YOD leading to inadequate advice/help". Two other themes characterize the problems experienced by YOD caregivers in the period prior to receiving a diagnosis: (6) "changes to the family member" and (7) "disrupted family life". These findings partly overlap with existing literature on YOD as well as on LOD, but there are also factors distinguishing the experiences of YOD caregivers from those of the elderly.

An additional possible barrier to obtaining a diagnosis in YOD is the occurrence of behavioral symptoms. Overall, behavioral changes were mentioned frequently $(57 \%)$. The same was true when looking only at the initial symptoms $(43 \%)$. The general idea that behavioral disturbances and problems in social functioning only present in later stages of $\mathrm{AD}^{2}$ may therefore be unwarranted. Misattribution of symptoms appears to be common in YOD and our findings suggest behavioral changes play an important role in this process. While in LOD behavioral symptoms trigger recognition of dementia ${ }^{21}$, in our group, caregivers related them to psychological causes.

Further factors leading to attributions to psychological causes were difficulties at work, dismissal from work or other potentially influential events, such as the caregiver becoming ill. Normalizing behavior by understanding it in relation to a stressful event is common before a dementia diagnosis is given, because in such events it is acceptable to behave differently ${ }^{14}$. Explaining symptoms in the light of problems at work is therefore obviously common in young people and is also demonstrated by the establishment of diagnoses of burnout. Although potentially leading to misinterpretation, it is important for caregivers to know about the work related problems of their family member. This helps them to become aware that something is wrong and decide to seek help. These findings suggest a role for employers and company doctors in facilitating recognition of dementia in younger people.

The denial of symptoms by the person with YOD was an additional factor 
making it hard for caregivers to deal with their changing family member and, in several cases, led to refusal and delay in help seeking. This finding is in line with other studies on dementia in different age groups ${ }^{11,13}$. Denial is common in dementia and may serve as a protective mechanism, especially when the concept of self is under threat, which is the case with the onset of dementia ${ }^{22}$. The social context, including the relationship with the partner is likely to have an impact on the way the person with early stage dementia understands what is happening ${ }^{22}$. From the present data it appears that, in the case of refusal to seek advice, someone other than the spouse can play a key role in persuading a person with YOD to seek medical advice. Also when the caregiver was not aware of changes in the patient, the social context played an important role in triggering this awareness, which has also been established in $\mathrm{LOD}^{14}$.

Other factors leading to the prolongation of the period prior to diagnosis were lack of responsiveness from GPs and the establishment of erroneous diagnoses leading to inadequate support or treatment. Therefore, not only GPs, but also therapists should have knowledge about YOD, enabling them to pick up early dementia signs. The referral trajectory was experienced as problematic and too long. The diagnosis came as a relief in some cases, illustrating the magnitude of the struggles the YOD caregivers experienced. In studies on LOD, similar reactions after diagnostic disclosure have been reported ${ }^{23,} 24$. In LOD, it has also been reported that caregivers experience resistance from physicians when trying to convince them something is wrong $^{24}$ and dementia appears to be commonly under-diagnosed ${ }^{25}$.

Besides the factors prolonging the period prior to diagnosis, several aspects have been identified impacting on the client system. Behavioral symptoms are one of the major risk factors for caregiver burden ${ }^{26,27}$ and are hard for caregivers to understand, even when a diagnosis has been given ${ }^{28}$. Our finding that behavioral symptoms had a high impact on the spousal relationship is in line with literature showing that behavioral problems independent of cognitive or functional status are associated with deterioration in the quality of the marital relationship. Apathy in particular appears to diminish the amount and reciprocity of interactions between spouses $^{29}$ and this was a relatively frequent symptom in the present study.

The other issues from the theme "disrupted family life", - e.g. problems with children and work and financial issues - are likely to be more specific to people with YOD because of their different phase in life compared to those with LOD. These problems have repeatedly been reported in the literature to be important within YOD families ${ }^{30}$. This finding is in line with a study on the impact of having a father with YOD, which showed that a delay in diagnosis and misdiagnosis instilled uncertainty and confusion in the children. The same study showed that diagnosis was important for acquiring the appropriate financial support ${ }^{31}$. Other studies have attempted to explore the unique challenges people with YOD and their caregivers experience. However, their main focus was not on the period prior to diagnosis ${ }^{8}$ or they focused only on the referral trajectory ${ }^{7}, 11$.

The present study provides the first detailed overview of the specific experi- 
ences of a unique sample of YOD caregivers in the period before diagnosis. The strength of qualitative research is that it is suitable to study complex issues, it illuminates context and identifies areas to be explored in future research. Combining this method with quantitative research methods provides us with a deeper understanding of the experiences of YOD caregivers as well as an indication of how these are distributed in the YOD group. Considering the fact that the analyses are mainly based on spontaneously mentioned problems, the reported percentages are most likely an underestimation of the total proportion of caregivers experiencing these problems.

Although qualitative research is interpretative in itself, researchers need to be reflexive on their own influence on data analysis. The strengths of this study are that separate analyses have been done by different researchers and there were continuous discussions between them, which is important for increasing theoretical sensitivity and the trustworthiness of the data ${ }^{18,20}$.

A limitation of the present study is that the "erroneous" diagnoses reported here sometimes actually represent comorbidity, the suspicion of a physician and, in one case, MCI. However, the study shows how these are experienced by caregivers and how symptoms are labelled by the medical profession. It should also be noted that we did not have access to all complete medical records. Portions of medical files were received from external hospitals in 37 cases.

In addition, the results are restricted to the YOD group because no comparison group was used. Therefore, it is not clear whether the themes revealed result in a longer delay in diagnosing YOD than LOD. Misattribution of symptoms for example is also common in LOD. The older people are, the more they tend to attribute memory problems to normal ageing. Furthermore, although older people may suspect dementia sooner, this does not necessarily mean they seek help earlier. Fear of facing the possibility of dementia has been found to delay help seeking ${ }^{13}$, which may be related to fear of admission to residential care. Likewise, being employed may facilitate recognition in younger people, because it is more evident there is a problem and more difficult to deny. Further, possible genetic links in presenile dementia and senile dementia with early onset, can make family members more conscious of symptoms ${ }^{32}$. It has been found that YOD patients were less severely impaired on presentation than LOD patients ${ }^{33}$.

However, the factors and dynamics causing the delay in obtaining a diagnosis are probably different in the YOD than in the LOD group, and the impact on family life seems to be higher in YOD than in LOD.

In conclusion, this study provides some insight into the factors prolonging the time to diagnosis. The denial and the refusal to seek help by the person with YOD, the misattribution of symptoms, professionals' inadequate help and faulty diagnoses are key issues for future support for YOD caregivers and should be studied further. Furthermore, YOD patients encounter a high number of problems in the period prior to diagnosis, which cause strain and insecurity in several life domains. These problems seem specifically related to the situation of people with YOD. Although a timely diagnosis is not always considered useful by $\mathrm{GPs}^{34,35}$, 
these findings show the specific importance for younger people to receive an earlier diagnosis. Knowing the nature/background of difficulties experienced may help caregivers and patients better understand and cope with their increasingly difficult situation; it may prevent loss of their jobs, divorce or disrupted relationships with children.

These findings underline the need for faster and more adequate support from healthcare professionals. It should be noted that establishing a dementia diagnosis will remain a challenge for clinicians until unique differentiating markers are found. The risk of erroneously diagnosing psychiatric conditions as dementia is also present, as shown in previous studies ${ }^{36,37}$. Recommendations, therefore, are mainly aimed at improving clinicians' alertness to the possibility of dementia at young age, improving responsiveness to individual needs and ensuring the regular follow-up of people presenting with cognitive and/or behavioral changes. In addition, society needs to be more sensitive about dementia and YOD symptoms. 


\section{References}

1. van Vliet D, de Vugt ME, Bakker C, et al. How do caregivers of early onset dementia patients perceive the period prior to diagnosis. In: Abstract book of the international meeting of the International Psychogeriatric Association: 26-29 September, 2010, Santiago de Compostela, 2010: 2.

2. Sampson EL, Warren JD, Rossor MN. Young onset dementia. Postgraduate Medical Journal 2004;80:125-139.

3. Mendez MF. The accurate diagnosis of early-onset dementia. International Journal of Psychiatry in Medicine 2006;36:401-412.

4. Bryan G, Martin CM. Unique problems of dementia in the younger patient. The Consultant Pharmacist 2005;20:468-479.

5. Kelley BJ, Boeve BF, Josephs KA. Cognitive and noncognitive neurological features of young-onset dementia. Dementia and Geriatric Cognitive Disorders 2009; $27: 564-571$.

6. Luscombe G, Brodaty H, Freeth S. Younger people with dementia: Diagnostic issues, effects on carers and use of services. International Journal of Geriatric Psychiatry 1998;13:323-330.

7. Williams T, Cameron I, Deardon T. From pillar to post-a study of younger people with dementia. Psychiatric Bulletin 2001;25:384-387.

8. Harris PB, Keady J. Living with early onset dementia: exploring the experience and developing evidence-based guidelines for practice. Alzheimer's Care Quarterly 2004;5:111-122.

9. Sperlinger D, Furst M. The service experiences of people with presenile dementia: A study of carers in one London borough. International Journal of Geriatric Psychiatry 1994;9:47-50.

10. Werner P, Stein-Shvachman I, Korczyn AD. Early onset dementia: clinical and social aspects. International Psychogeriatrics 2009:1-6.

11. Newens AJ, Forster DP, Kay DW. Referral patterns and diagnosis in presenile Alzheimer's disease: implications for general practice. The British Journal of General Practice 1994;44:405-407.

12. Leung KK, Finlay J, Silvius JL, et al. Pathways to diagnosis: exploring the experiences of problem recognition and obtaining a dementia diagnosis among AngloCanadians. Health 85 Social Care in the Community 2011;Epub ahead of print.

13. Clark PC, Kutner NG, Goldstein FC, et al. Impediments to timely diagnosis of Alzheimer's disease in African Americans. Journal of the American Geriatrics Society 2005;53:2012-2017.

14. Krull AC. First signs and normalizations: Caregiver routes to the diagnosis of Alzheimer's disease. Journal of Aging Studies 2005;19:407-417.

15. van Vliet D, Bakker C, Koopmans RT, Vernooij-Dassen MJ, Verhey FR, de Vugt ME. Research protocol of the NeedYD-study (Needs in Young onset Dementia): a prospective cohort study on the needs and course of early onset dementia. BMC Geriatrics 2010;10:13. 
16. Folstein MF, Folstein SE, McHugh PR. Mini-mental state: A practical method for grading the cognitive state of patients for the clinician. Journal of Psychiatric Research 1975;12:189-198.

17. Muhr T. ATLAS.ti 5.0 [Version 5:]. Berlin, Germany: ATLAS.ti Scientific Software Development GmbH. Available from http://www.atlasti.com/. 2004.

18. Corbin J, Strauss A. Grounded Theory Research: Procedures, Canons, and Evaluative Criteria: Springer Science \& Business Media B.V., 1990.

19. Glaser BG, Strauss AL. The discovery of grounded theory: strategies for qualitative research. Chicago: Aldine Pub. Co, 1967.

20. Endacott R. Clinical research 4: qualitative data collection and analysis. International Emergency Nursing 2008;16:48-52.

21. Eustace A, Bruce I, Coen R, et al. Behavioural disturbance triggers recognition of dementia by family informants. International Journal of Geriatric Psychiatry 2007;22:574-579.

22. Clare L. Developing awareness about awareness in early-stage dementia: The role of psychosocial factors. Dementia: The International Journal of Social Research and Practice 2002;1:295-312.

23. Derksen E, Vernooij-Dassen M, Gillissen F, Rikkert MO, Scheltens P. Impact of diagnostic disclosure in dementia on patients and carers: Qualitative case series analysis. Aging \& Mental Health 2006;10:525-531.

24. Connell CM, Boise L, Stuckey JC, Holmes SB, Hudson ML. Attitudes toward the diagnosis and disclosure of dementia among family caregivers and primary care physicians. Gerontologist 2004;44:500-507.

25. Iliffe S, Robinson L, Brayne C, et al. Primary care and dementia: 1. diagnosis, screening and disclosure. International Journal of Geriatric Psychiatry 2009;24:895-901.

26. Ballard C, Lowery K, Powell I, O'Brien J, James I. Impact of behavioral and psychological symptoms of dementia on caregivers. International Psychogeriatrics 2000;12:93-105.

27. de Vugt ME, Riedijk SR, Aalten P, Tibben A, van Swieten JC, Verhey FR. Impact of behavioural problems on spousal caregivers: a comparison between Alzheimer's disease and frontotemporal dementia. Dementia and Geriatric Cognitive Disorders 2006;22:35-41.

28. Paton J, Johnston K, Katona C, Livingston G. What causes problems in Alzheimer's disease: Attributions by caregivers. A qualitative study. International Journal of Geriatric Psychiatry 2004;19:527-532.

29. de Vugt ME, Stevens F, Aalten P, et al. Behavioural disturbances in dementia patients and quality of the marital relationship. International Journal of Geriatric Psychiatry 2003;18:149-154.

30. van Vliet D, de Vugt ME, Bakker C, Koopmans RT, Verhey FR. Impact of early onset dementia on caregivers: a review. International Journal of Geriatric Psychiatry 2010;25:1091-1100. 
31. Allen J, Oyebode JR, Allen J. Having a father with young onset dementia: The impact on well-being of young people. Dementia: The International Journal of Social Research and Practice 2009;8:455-480.

32. Miyoshi K. What is early onset dementia? Psychogeriatrics 2009;9:67-72.

33. McMurtray A, McMurtray A, Clark DG, Christine D, Mendez MF. Early-Onset Dementia: Frequency and Causes Compared to Late-Onset Dementia. Dementia and Geriatric Cognitive Disorders 2006;21:59-64.

34. Hansen EC, Hughes C, Routley G, Robinson AL. General practitioners' experiences and understandings of diagnosing dementia: factors impacting on early diagnosis. Social Science \& Medicine 2008;67:1776-1783.

35. Cahill S, Clark M, O'Connell H, Lawlor B, Coen RF, Walsh C. The attitudes and practices of general practitioners regarding dementia diagnosis in Ireland. International Journal of Geriatric Psychiatry 2008;23:663-669.

36. Ron MA, Toone BK, Garralda ME, Lishman WA. Diagnostic accuracy in presenile dementia. The British Journal of Psychiatry 1979;134:161-168.

37. Marsden CD, Harrison MJ. Outcome of investigation of patients with presenile dementia. British Medical Journal 1972;2:249-252. 


\section{Chapter 6}

\section{Prevalence of neuropsychiatric symptoms in young-onset compared to late-onset Alzheimer's disease}

Based on: van Vliet D, de Vugt ME, Aalten P, Bakker C, Pijnenburg YAL, Vernooij-Dassen MJFJ, Koopmans RTCM, Verhey FRJ (2012) Prevalence of neuropsychiatric symptoms in young-onset compared to late-onset Alzheimer's disease. Part 1: findings of the two-year longitudinal NeedYD-study. Dementia and Geriatric Cognitive Disorders Accepted for publication. 


\section{Abstract}

Background/aims Knowledge about neuropsychiatric symptoms in young onset Alzheimer's disease (YO-AD) is scarce, but essential to establish a prognosis and plan care for YO-AD patients. The aim of this study is to assess frequency parameters of neuropsychiatric symptoms in YO-AD over two years and investigate whether there are differences compared with late onset Alzheimer's disease (LO-AD).

Methods 98 YO-AD-and 123 LO-AD-patients and caregivers from two prospective cohort studies were included and assessed every six months for two years, using the Neuropsychiatric Inventory (NPI) to evaluate neuropsychiatric symptoms.

Results Over the course of two years, the incidence, prevalence and persistence of neuropsychiatric symptoms were in general lower in YO-AD than in LO-AD, specifically for delusions, agitation, depression, anxiety, apathy, irritability and aberrant motor behavior. Frequency of individual symptoms showed large variability in both groups. Within the group of YO-AD patients, apathy, was the most prevalent symptom.

Conclusion Neuropsychiatric symptoms, notably apathy, should be paid appropriate attention to in the diagnosis and treatment of YO-AD patients. Further research is needed to gain insight into the mechanisms underlying the differences in neuropsychiatric symptoms between YO-AD and LO-AD. 


\subsection{Introduction}

Neuropsychiatric symptoms in dementia are a significant contributor to caregiver burden $^{1}$; they reduce the quality of life of patients ${ }^{2}$ and are the main cause of institutionalization ${ }^{3,4}$. Nearly every patient with dementia develops neuropsychiatric symptoms $s^{5,6}$, and these problems usually continue over longer periods of time $^{5,7}$. The evaluation of neuropsychiatric symptoms in patients with the most common cause of dementia, Alzheimer's disease (AD), has mainly focused on older patients. Hence, little is known about the neuropsychiatric profile in young-onset $\mathrm{AD}(\mathrm{YO}-\mathrm{AD})$ patients, with an onset before age 65. Acquiring more insight into the nature and course of neuropsychiatric symptoms in YO-AD is important to direct treatment, to provide psycho-education and to plan care and support for patients and their caregivers. This information will contribute to the quality of life of both patients and caregivers and may even postpone institutionalization, which comprises the greatest component of long-term care expenditures ${ }^{8}$.

It has been suggested that younger dementia patients may display more neuropsychiatric problems, specifically affective symptoms, because a dementia diagnosis at a young age can result in more unexpected loss of independence and isolation $^{9,10}$. However, the few studies comparing YO-AD with late onset AD (LO-AD) have shown equivocal results. One study did not find any differences between these two groups in the prevalence of depression and anxiety, while they did find fewer and less severe delusions, hallucinations, agitation, disinhibition, aberrant motor behavior and total problem behavior in $\mathrm{YO}-\mathrm{AD}$ than in $\mathrm{LO}-\mathrm{AD}^{11}$. By contrast, another study found more inappropriate behavior in YO-AD compared with LO-AD ${ }^{12}$. Further, the severity of depressive symptoms was found to be higher in YO-AD in one study ${ }^{13}$ and the prevalence lower in another ${ }^{14}$. These inconsistencies are difficult to interpret because of the heterogeneity of these studies. Additionally, the reported studies included rather small samples of YO-AD patients (ranging from 21 to 51).

The current study is the first to assess the point prevalence, cumulative prevalence, incidence and persistence of a broad range of neuropsychiatric symptoms in YO-AD patients over two years in comparison with LO-AD. Differences in frequency and patterns of neuropsychiatric symptoms between $\mathrm{YO}-\mathrm{AD}$ and $\mathrm{LO}-\mathrm{AD}$ would suggest the existence of age-related subgroups or a strong influence of different occupational and social circumstances on the neuropsychiatric manifestations. Based on the hypothesis that a dementia diagnosis at a younger age is more unexpected and has a greater impact, we expect a higher prevalence of depression and anxiety in YO-AD than in LO-AD. 


\subsection{Methods}

\section{Subjects}

This study is part of a Dutch prospective cohort study, the NeedYD-study (Needs in Young onset Dementia), which has been described in more detail elsewhere ${ }^{15}$. In short, 215 participants with young-onset dementia (YOD) with an age of onset before 65 were recruited. Assessments with patients and their primary caregivers were performed at baseline and repeated every six months for two years. Patients were sampled from specialized daycare facilities $(n=115)$, consecutive referrals from University Medical Centers in Maastricht and Amsterdam $(\mathrm{n}=56)$, regional hospitals $(\mathrm{n}=10)$ or regional community mental health services $(\mathrm{n}=20)$, or they individually applied to participate $(\mathrm{n}=14)$. Inclusion criteria were age at onset younger than 65 (thus, age at inclusion could be over 65), a dementia diagnosis and availability of an informant who has regular contact with the patients (defined as minimally once per week). The diagnosis of dementia was made according to the criteria from the $4^{\text {th }}$ edition of the Diagnostic and Statistical Manual of Mental Disorders, text revision $(2000)^{16}$ and the Dutch consensus guidelines ${ }^{17}$, which use internationally accepted criteria for diagnosing dementia subtypes ${ }^{18-22}$. Clinical diagnoses were checked against the clinical patient files for each patient. Exclusion criteria were (1) lack of informed consent of the participant; (2) living in a nursing home; or (3) dementia caused by HIV, traumatic brain injury, Down's syndrome, Huntington's disease or alcohol-related dementia. The latter disorders were excluded as they were not part of the traditional memory clinic populations, and they are accompanied by specific social aspects that would make the two cohorts not comparable.

A comparison group of patients with LOD was selected from a 2-year followup cohort study with LOD patients, the MAASBED study (MAAstricht Study of Behavior in Dementia) ${ }^{23}$, which had a similar design, assessment measures and diagnostic criteria as the NeedYD-study. In the MAASBED study, patients with dementia were included irrespective of the diagnostic subtype. Patients from the MAASBED study were consecutively enrolled from the memory clinic of the Maastricht University Medical Center or the Community Mental Health Care Team (RIAGG) in Maastricht. In the present study, patients were selected from these cohorts if they met the criteria for $\mathrm{AD}$, if an MMSE score was available and if the informant was an informal caregiver to obtain homogeneous comparison groups. Because we adopted these inclusion criteria, the original samples of $142 \mathrm{YO}-\mathrm{AD}$ and $126 \mathrm{LO}-\mathrm{AD}$ patients were reduced in sample size, due to missing MMSE scores. The MAASBED cohort originally included 20 YO-AD patients who were added to the YO-AD group. Based on these criteria, 98 patients with young onset $\mathrm{AD}(\mathrm{YO}-\mathrm{AD})$ and 123 patients with late onset $\mathrm{AD}(\mathrm{LO}-\mathrm{AD})$ were included in the present study. The study protocol was approved by the Medical Ethics Committee of the Maastricht University Medical Center. Written informed consent of all subjects or their legal representatives was obtained prior to the investigation. 


\section{Assessment of neuropsychiatric symptoms}

Neuropsychiatric symptoms were assessed by means of the Neuropsychiatric Inventory $(\mathrm{NPI})^{24}$. The NPI is a structured interview with the primary caregiver and is the most widely used instrument to evaluate neuropsychiatric problems in dementia, assessing ten neuropsychiatric and two neurovegetative symptoms of which the frequency and severity are rated on a five- and four-point Likert scale, respectively. These scores are multiplied to obtain total item scores ranging from $0-12$. The total NPI score is the summed symptom score. The reliability and validity of the Dutch version of the NPI have been demonstrated ${ }^{25}$.

\section{Assessment of secondary outcome measures}

Age, sex, level of education (ranging from (1) primary school to (8) university degree), disease duration (years from symptom onset to study entry) and patients' medication use were gathered from the primary caregivers. The Global Deterioration Scale (GDS) was used as a measure of dementia severity. Cognitive functioning was measured using the Mini Mental State Examination (MMSE) ${ }^{26}$. The reliability and validity of these instruments have been established ${ }^{27}$.

\section{Analysis}

Analyses were performed using the Statistical Package for Social Sciences (SPSS) version 19.0. Differences between the YO-AD and LO-AD group in baseline characteristics were tested with t-tests for continuous and $\chi^{2}$-tests for categorical variables. If the assumptions for parametric testing were not met, Mann-Whitney $\mathrm{U}$ tests were performed instead.

We investigated the following frequency parameters: point prevalence, cumulative prevalence, cumulative incidence and persistence of symptoms, all calculated using SPSS macro syntax. The presence of a symptom was expressed as an NPI item score $>3$, indicating a clinically relevant neuropsychiatric symptom ${ }^{5}$. The cumulative prevalence was defined as the proportion of patients developing a specific symptom on at least one assessment over the 2-year study period. The cumulative incidence was rated as the proportion of patients who were symptom free at baseline but developed the specific symptom at follow-up assessments. The persistence of symptoms was calculated as the percentage of patients showing a specific symptom on either two, three, four or five successive assessments, regardless of the time of first manifestation of the symptom. The cumulative persistence was defined as the percentage of patients who displayed a specific symptom during at least two subsequent assessments (i.e., the summed percentage of previously mentioned persistence scores), regardless of the time of first manifestation of the symptom. These frequency parameters were compared between YO-AD and LO$\mathrm{AD}$ patients who completed the 2-year follow-up using logistic regression analyses, corrected for gender, education and disease duration. 


\subsection{Results}

\section{Sample characteristics}

In total, 221 patients (98 YO-AD, $123 \mathrm{LO}-\mathrm{AD}$ ) were included in the study, of whom $147(66.5 \%)$ (79 YO-AD, 68 LO-AD) could be followed up for all assessments during the 2-year period. During this period, 17 participants refused (9 YO$\mathrm{AD}, 8 \mathrm{LO}-\mathrm{AD})$ to participate, 24 participants were lost to follow-up (4 YO-AD, 20 LO-AD) and 33 patients died (6 YO-AD, 27 LO-AD). The completers did not differ from non-completers in terms of gender $\left(\chi^{2}(1)=0.059, p=.807\right)$, education $\left(\chi^{2}(1)=2.329, p=.312\right)$, stage of dementia $\left(\chi^{2}(1)=5.217, p=.074\right)$, overall medication use (using any of the psychotropic drugs as shown in table 6.1) $\left(\chi^{2}(1)=2.932, p=.087\right)$ or NPI total score at baseline $(t(215)=0.651, p=.516)$. Non-completers were older $(t(219)=3.310, p=.001)$, had a lower MMSE score $(t(219)=-2.205, p=.029)$ and had a shorter disease duration $(U=3820.0, p=$ $.004)$.

Table 6.1 shows the baseline characteristics of the total group and the patients with complete follow-up. In the total YO-AD group, patients were on average more frequently male, with a higher education, longer disease duration and more advanced stage of dementia than the total LO-AD group. Furthermore, patients in the total YO-AD group used less anxiolytics and more antidementia medications. These findings were similar for patients who completed the study, except that the disease severity was similar between the total YO-AD and LO-AD groups.

\section{Point prevalence}

Table 6.2 shows the point prevalence (prevalence at each assessment) of the NPI symptoms separated for the YO-AD and LO-AD group who completed follow-up. Overall, neuropsychiatric symptoms were less common in the YO-AD group. In $54.4 \%$ of YO-AD patients and $80.9 \%$ of LO-AD patients, at least one neuropsychiatric symptom was present at baseline, which then increased in both groups during the study period to $74.7 \%$ in YO-AD and to $88.2 \%$ in LO-AD. Apathy $(38.0 \%)$ and aberrant motor behavior $(25.3 \%)$ were the most common symptoms in YO-AD at study onset, showing an increase in prevalence over time. Depression $(45.6 \%)$ and apathy $(39.7 \%)$ were the most prevalent at study onset in LO-AD, with depression showing a decrease over time, and apathy, an increase. The next most frequent symptoms in YO-AD were irritability, agitation and eating changes. In LO-AD, delusions, agitation, anxiety, irritability, aberrant motor behavior and eating changes were the next most prevalent. In both groups, apathy and aberrant motor behavior showed the highest increase in prevalence over time. 


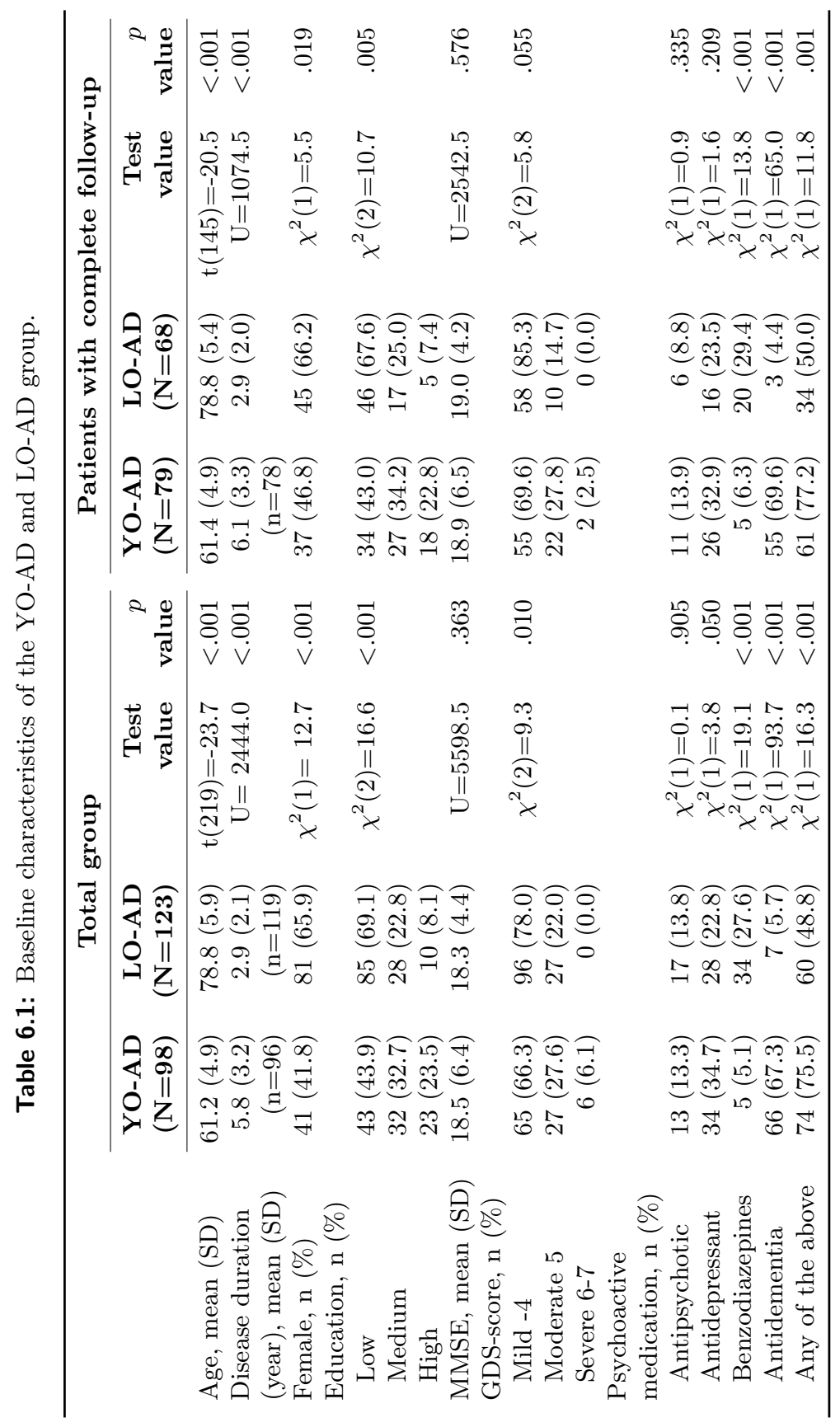


Table 6.2: Point prevalence of neuropsychiatric symptoms for the YO-AD and LO-AD group.

\begin{tabular}{|c|c|c|c|c|c|c|c|c|c|c|}
\hline \multirow[b]{2}{*}{ Prevalence (\%) } & \multicolumn{5}{|c|}{ YO-AD $(n=79)$} & \multicolumn{5}{|c|}{ LO-AD $(n=68)$} \\
\hline & T0 & $\mathrm{T} 1$ & $\mathrm{~T} 2$ & T3 & $\mathrm{T} 4$ & T0 & $\mathbf{T 1}$ & $\mathrm{T} 2$ & T3 & $\mathbf{T 4}$ \\
\hline Delusions & 7.6 & 3.8 & 3.8 & 6.3 & 3.8 & 23.5 & 25.0 & 25.0 & 35.3 & 20.6 \\
\hline Hallucinations & 2.5 & 2.5 & 2.5 & 2.5 & 5.1 & 5.9 & 7.4 & 10.3 & 8.8 & 8.8 \\
\hline Agitation & 21.5 & 13.9 & 15.2 & 12.7 & 15.2 & 25.0 & 25.0 & 26.5 & 20.6 & 23.5 \\
\hline Depression & 16.5 & 17.7 & 19.9 & 15.2 & 12.7 & 45.6 & 32.4 & 29.4 & 27.9 & 26.5 \\
\hline Anxiety & 16.5 & 11.4 & 13.9 & 17.7 & 8.9 & 26.5 & 20.6 & 23.5 & 17.6 & 19.1 \\
\hline Euphoria & 2.5 & 3.8 & 5.1 & 6.3 & 1.3 & 2.9 & 1.5 & 2.9 & 2.9 & 2.9 \\
\hline Apathy & 38.0 & 35.4 & 49.4 & 41.8 & 51.9 & 39.7 & 47.1 & 66.2 & 50.0 & 66.2 \\
\hline Disinhibition & 11.4 & 8.9 & 7.6 & 13.9 & 8.9 & 5.9 & 7.4 & 10.3 & 11.8 & 17.6 \\
\hline Irritability & 24.1 & 15.2 & 19.0 & 17.7 & 12.7 & 26.5 & 29.4 & 30.9 & 30.9 & 26.5 \\
\hline Aberrant motor behavior & 25.3 & 25.3 & 39.2 & 36.7 & 41.8 & 22.1 & 38.2 & 47.1 & 44.1 & 45.6 \\
\hline Nighttime behavior & 7.6 & 8.9 & 16.5 & 10.1 & 15.2 & 16.2 & 14.7 & 13.2 & 5.9 & 11.8 \\
\hline Eating changes & 20.3 & 24.1 & 15.2 & 29.1 & 24.1 & 25.0 & 17.6 & 26.5 & 11.8 & 22.1 \\
\hline NPI total & 54.4 & 63.3 & 79.7 & 72.2 & 74.7 & 80.9 & 79.4 & 92.6 & 83.8 & 88.2 \\
\hline
\end{tabular}

\section{Cumulative incidence, prevalence and persistence}

In table 6.3, frequency estimates of neuropsychiatric symptoms are shown for the members of the YO-AD and LO-AD group who competed the follow-up period. The cumulative incidence and prevalence were overall lower in the YO-AD group. The incidence rate of delusions was over three times lower $(\mathrm{OR}=0.16,95 \% \mathrm{CI}=0.06$ $0.42, \mathrm{p}<.001)$ in $\mathrm{YO}-\mathrm{AD}$, and the cumulative incidences of agitation $(\mathrm{OR}=0.34$, $95 \% \mathrm{CI}=0.13-0.91, \mathrm{p}=.031)$, apathy $(\mathrm{OR}=0.07,95 \% \mathrm{CI}=0.01-0.37, \mathrm{p}=.002)$ and irritability $(\mathrm{OR}=0.26,95 \% \mathrm{CI}=0.09-0.75, \mathrm{p}=.012)$ were also significantly lower in YO-AD than in LO-AD. In the YO-AD group $78.8 \%$ of symptom free patients $(\mathrm{n}=33)$ at baseline developed a clinically relevant symptom during the two-year period and in the LO-AD group $100.0 \%$ of symptom free patients $(n=13)$. The two-year prevalence of delusions $(\mathrm{OR}=0.17,95 \% \mathrm{CI}=0.07-0.40, \mathrm{p}<.001)$, agitation, $(\mathrm{OR}=0.45,95 \% \mathrm{CI}=0.20-0.99, \mathrm{p}=.049)$, apathy $(\mathrm{OR}=0.12,95 \% \mathrm{CI}=0.03-0.42$, $\mathrm{p}=.001)$ and irritability $(\mathrm{OR}=0.35,95 \% \mathrm{CI}=0.15-0.79, \mathrm{p}=.011)$ were also significantly lower in YO-AD. In addition, significantly fewer patients in the YO-AD group $(40.5 \%)$ had depressive symptoms $(\mathrm{OR}=0.23,95 \% \mathrm{CI}=0.10-0.53, \mathrm{p}=.001)$ or anxiety $(40.5 \%)(\mathrm{OR}=0.40,95 \% \mathrm{CI}=0.18-0.89, \mathrm{p}=.025)$ compared with the LOAD group (72.1\% for depression, $55.9 \%$ for anxiety). In both groups, apathy was among the most and euphoria and hallucinations were among the least incident and prevalent symptoms over the two year period. 


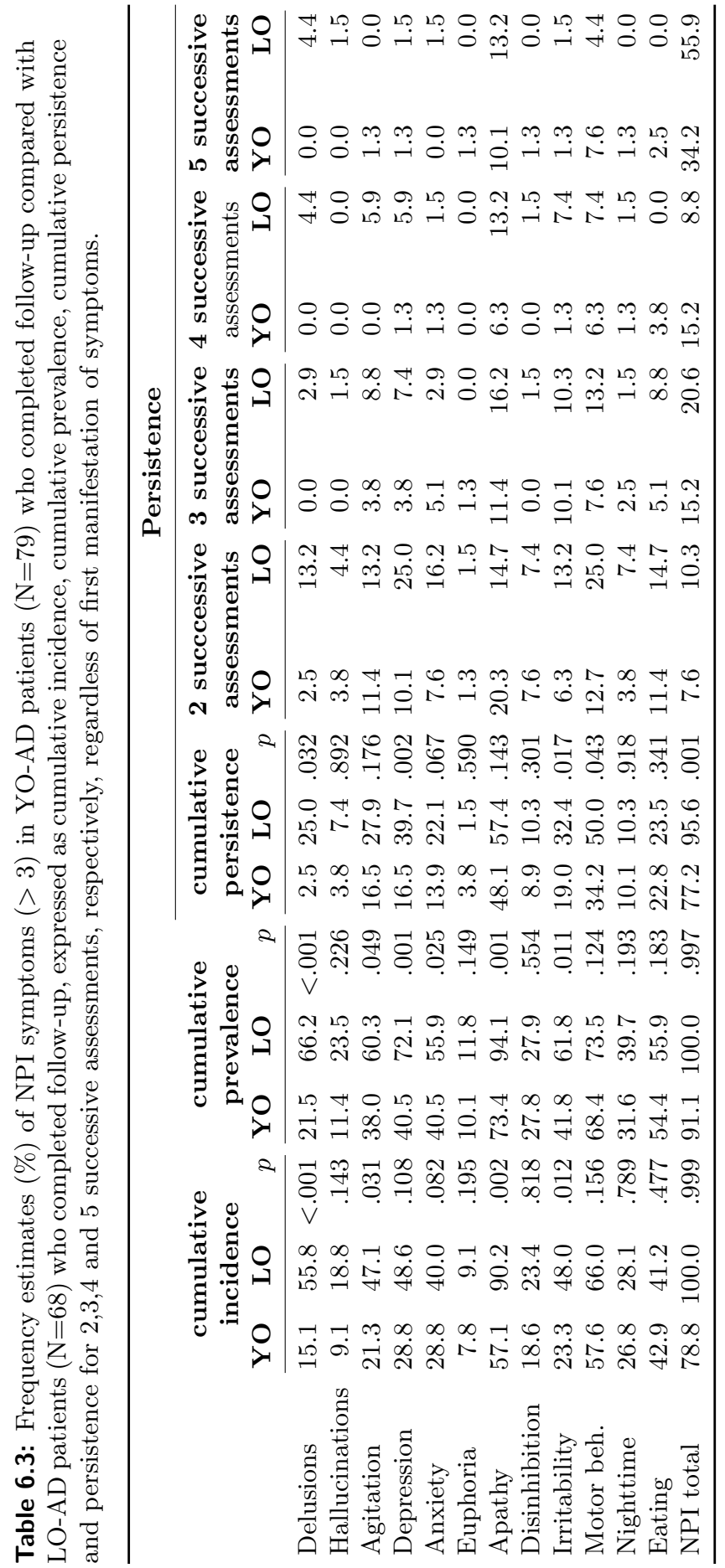


Table 6.4: Point prevalence of neuropsychiatric symptoms for the YO-AD and LO-AD group.

\begin{tabular}{lllll}
\hline & YO-AD & & LO-AD & \\
\hline 1 & Apathy & 73.4 & Apathy & 94.1 \\
2 & Aberrant motor & 68.4 & Aberrant motor & 73.5 \\
3 & Eating changes & 54.4 & Depression & 72.1 \\
4 & Irritability & 41.8 & Delusions & 66.2 \\
5 & Depression & 40.5 & Irritability & 61.8 \\
6 & Anxiety & 40.5 & Agitation & 60.3 \\
7 & Agitation & 38.0 & Eating changes & 55.9 \\
8 & Nighttime behavior & 31.6 & Anxiety & 55.9 \\
9 & Disinhibition & 27.8 & Nighttime behavior & 39.7 \\
10 & Delusions & 21.5 & Disinhibition & 27.9 \\
11 & Hallucinations & 11.4 & Hallucinations & 23.5 \\
12 & Euphoria & 10.1 & Euphoria & 11.8 \\
\hline
\end{tabular}

Further, the number of patients showing neuropsychiatric symptoms on two or more subsequent assessments was significantly lower in the YO-AD group $(77.2 \%$ versus $95.6 \%$ in $\mathrm{LO}-\mathrm{AD}(\mathrm{OR}=0.08,95 \% \mathrm{CI}=0.02-0.34, \mathrm{p}=.001)$. Just over half of the LO-AD patients and around one-third of the YO-AD patients displayed one or more neuropsychiatric symptom during all five assessments of the study period. However, individual symptoms did not persist for longer periods in most patients. Delusions $(\mathrm{OR}=0.71,95 \% \mathrm{CI}=0.03-0.86, \mathrm{p}=.032)$, depression $(\mathrm{OR}=0.22$, $95 \% \mathrm{CI}=0.08-0.58, \mathrm{p}=.002)$, irritability $(\mathrm{OR}=0.30,95 \% \mathrm{CI}=0.11-0.81, \mathrm{p}=.017)$ and aberrant motor behavior $(\mathrm{OR}=0.43,95 \% \mathrm{CI}=0.19-0.97, \mathrm{p}=.043)$ were less persistent in YO-AD than in LO-AD. Over the whole study period, again apathy was the most persistent and euphoria and hallucinations were the least persistent symptoms in both groups.

\section{Ranking of frequency parameters}

To gain further insight into the possible differences in the profile of neuropsychiatric symptoms between $\mathrm{YO}-\mathrm{AD}$ and $\mathrm{LO}-\mathrm{AD}$, the frequency estimates were ranked in descending order for each group. The profiles for the cumulative prevalence estimates were broadly similar between groups (table 6.4). The largest differences in rank between groups were found for eating changes, which was one of the most frequent symptoms in $\mathrm{YO}-\mathrm{AD}$, and delusions, which was one of the least frequent symptoms in this group; by contrast, in the LO-AD group, a reverse pattern was present. Similar results were obtained for cumulative incidence and persistence but were not shown to aid in clarity. 


\subsection{Discussion}

In this study, the frequency of neuropsychiatric symptoms in YO-AD was investigated longitudinally for the first time and was compared with LO-AD. Over the course of two years, the incidence, prevalence and persistence of neuropsychiatric symptoms were overall lower in YO-AD than in LO-AD. Significantly less YO-AD patients who were symptom free at baseline developed delusions, agitation, apathy and irritability. These symptoms, as well as depression and anxiety also appeared to be overall less common over the whole study-period. In addition, there were less YO-AD patients who displayed delusions, depression, irritability, aberrant motor behavior or any symptom on two or more consecutive assessments. Furthermore, the frequency parameters of individual symptoms showed large variability in both groups. The profiles of neuropsychiatric symptoms were roughly similar between groups, but eating changes and delusions ranked differently in both groups.

\section{Overall neuropsychiatric symptoms}

In contrast to our expectations, the YO-AD group showed overall less neuropsychiatric symptoms both at baseline, and during the follow-up period of two years, even though disease duration was longer in YO-AD. In addition, a higher proportion of YO-AD patients, were symptom free at baseline (41.8\%, versus $19 \%)$, and during the two-year period (10\% versus $0 \%$ ) although this difference was not significant. Persistence was significantly lower in YO-AD, with less patients displaying symptom(s) on two or more consecutive assessments. Around one third of YO-AD patients showed neuropsychiatric symptoms the entire study period versus approx. $55 \%$ in LO-AD. We therefore cannot conclude that neuropsychiatric symptoms are overall chronically present, contrary to other studies on $\mathrm{LOD}^{5}, 28,29$.

Previous studies on YO-AD reported higher prevalence rates of neuropsychiatric symptoms, i.e. $45 \%$ for depression, $25 \%$ for anxiety ${ }^{30}, 37.5 \%$ for delusions and $28.1 \%$ for hallucinations ${ }^{31}$, but these were non-comparative studies. Although difficult to compare, their higher prevalence rates may be due to the smaller sample size (i.e. 32 and 39), different assessment methods or to the fact that they also included institutionalized patients. This may indicate that the prevalence of neuropsychiatric symptoms in YO-AD shows a larger increase over the course of the illness than in LO-AD, resulting in more neuropsychiatric symptoms in the later stages of the dementia. Our findings are indeed suggestive of a higher increase of neuropsychiatric symptoms in YO-AD, with a $20 \%$ point prevalence increase in $\mathrm{YO}-\mathrm{AD}$ and a $7 \%$ increase in LO-AD over two years.

Our results did support the only similar comparative study to date of Toyota et al. They also only included outpatients and reported overall lower prevalence rates of neuropsychiatric symptoms in YO-AD than in LO-AD. In line with their study we showed delusions and anxiety to have lower prevalence rates. However, contrary to their study, we did not find significant differences between groups for hallucinations and disinhibition, while we did find differences for agitation, 
depression, apathy and irritability. These inconsistencies may result from our use of cumulative prevalence over a 2-year period, while the other authors used data from a single time point with less stringent NPI symptom criteria ( $>0$ instead of $>3)$.

\section{Neuropsychiatric profile}

The fact that apathy was the most common and euphoria the least common symptom in $\mathrm{YO}-\mathrm{AD}$, is consistent with previous findings in both YOD and $\mathrm{LOD}^{5}, 11,32$. Because apathy is one of the most burdensome neuropsychiatric problems for LOD caregivers $^{33}$ and the one with the strongest impact on the spousal relationship ${ }^{34}$, its impact should be investigated in YOD as well. The neuropsychiatric profile of YO-AD showed a relative lower frequency of delusions and a higher frequency of eating changes in this group.

We found an increasing point prevalence of apathy and aberrant motor behavior in YO-AD as well as LO-AD, consistent with previous studies on $\mathrm{LOD}^{5,32}$. The point prevalence of depression showed a stable course in YO-AD but decreased in LO-AD. A decrease of depression in LOD has been previously found ${ }^{5,35}$ and may be explained by a worsening ability of patients to communicate their feelings. Depressive symptoms may also decrease because of decreasing levels of awareness as the disease progresses. The higher awareness of YO-AD patients over time ${ }^{36}$ may cause a milder decrease of depression in this group.

\section{Explaining differences between YO-AD and LO-AD}

An explanation for the lower frequency parameters in YO-AD may be the presence of a specific subgroup of YO-AD patients. Approximately one third of YO-AD patients seem to present with an atypical non-memory phenotype ${ }^{37}$. This phenotype has been associated with less severe atrophy of the medial temporal lobe ${ }^{38}$ and the absence of a homozygous $\varepsilon 4 / \varepsilon 4$ genotype. Neuropsychiatric symptoms, such as wandering, agitation, aggression and psychosis ${ }^{39,40}$ as well as $\beta$ amyloid deposition in frontal cortical areas ${ }^{41}$ seem related to carrying the APOE $\varepsilon 4$ allele, and psychotic symptoms seem related to medial temporal lobe pathology ${ }^{38}$. These associations might explain why several neuropsychiatric symptoms were less frequent in the YO-AD group.

In addition, several alternative explanations for our results need to be discussed. For instance, factors associated with psychosis that are commonly absent at younger ages, such as vision loss and medical causes ${ }^{42}$, may explain the lower occurrence of delusions and hallucinations in YO-AD. Similarly, contextual factors related to older age, such as death of a loved one and physical disability ${ }^{43}$, may increase the risk for depression in this group. Furthermore, the higher levels of depression in LO-AD may relate to the higher prevalence of cerebrovascular pathology in this group ${ }^{44}$ because these factors seem to play a role in developing depression $^{45,46}$. 
The lower frequency of depression in the YO-AD group may have resulted in lower frequencies of irritability and apathy because these symptoms can be viewed as symptoms of depression ${ }^{47-49}$. The lower frequency of apathy in YO-AD is an interesting finding in light of our previous study that showed levels of awareness to be higher in $\mathrm{YO}-\mathrm{AD}^{36}$. Apathy seems less common at younger ages and in $\mathrm{AD}$ patients with high levels of awareness ${ }^{50,51}$. This finding may be related to a specific pattern of neuropathology; for example, both apathy and anosognosia have been related to frontal lobe dysfunction. However, patients with high levels of awareness may also be better at adjusting their activities to changes in level of functioning ${ }^{50}$ and staying motivated to engage in activities; hence, they may show lower levels of apathy.

\section{Strengths and limitations}

The current study is the first longitudinal comparison of the frequency estimates of neuropsychiatric symptoms between YO-AD and LO-AD in a large sample. The follow-up period was long, and we employed commonly accepted methods for assessing neuropsychiatric symptoms. However, some potential limitations need to be considered. The YO-AD and LO-AD samples came from different studies using slightly different recruitment strategies. While both were sampled from memory clinics and institutes for community mental health using essentially the same design, assessment measures and diagnostic criteria, only the MAASBED study included consecutive referrals after diagnosis, while the NeedYD-study also included patients using specialized daycare. One may argue that the depression scores in $\mathrm{YO}-\mathrm{AD}$ were lower because these patients were seen longer after diagnosis than the LO-AD group. However, when re-analyzing the data of the YO-AD patients (not reported here) who were included in the study shortly after diagnosis $(\mathrm{n}=50)$, this argument was not supported. In addition, we did not correct for medication use at baseline, changes in medication over time or the possible use of psychosocial interventions. The LO-AD patients were drawn from a historical cohort, possibly explaining differences in medication prescription. The lower prevalence of behavioral symptoms in the YO-AD group may be partly explained by the higher frequency of antidementia medication use in this group, although cholinesterase inhibitors only have a modest positive effect on behavioral disturbances ${ }^{52-54}$. In addition, more patients in the LO-AD group used benzodiazepines, which may have reduced anxiety and sleeping disturbances in this group ${ }^{55}$, but this would mean an even larger difference between groups than currently found. It should be noted that benzodiazepine use has also been associated with a more rapid increase in NPI-total ${ }^{56}$. The sample characteristics also limit generalizability to the entire YO-AD and LO-AD population. 


\section{Implications}

Our hypothesis that the psychosocial context of YO-AD leads to a higher frequency of neuropsychiatric symptoms, in particular depression and anxiety was not confirmed. Instead, these symptoms were unexpectedly lower in YO-AD, which may be due to biological differences between YO-AD and LO-AD. Further research is needed to gain more insight into the mechanisms underlying these differences between groups. In particular, research should focus on the identification of subtypes of YO-AD, which eventually could lead to the development of targeted interventions.

Still, neuropsychiatric symptoms should be paid appropriate attention to in the diagnosis, treatment, support and psycho-education of YO-AD patients and caregivers, especially since previous research indicated that YOD caregivers perceive greater difficulties due to neuropsychiatric disturbances than do LOD caregivers ${ }^{57}$. Similar to LO-AD, apathy is the most important symptom to focus on in YO-AD. Future studies should focus on the impact of neuropsychiatric symptoms, notably apathy, on YOD caregivers as well as on children of YOD patients, because this would yield important information on how to provide them with adequate support. 


\section{References}

1. Rocca P, Leotta D, Liffredo C, et al. Neuropsychiatric symptoms underlying caregiver stress and insight in Alzheimer's disease. Dementia and Geriatric Cognitive Disorders 2010;30:57-63.

2. Samus QM, Rosenblatt A, Steele C, et al. The Association of Neuropsychiatric Symptoms and Environment With Quality of Life in Assisted Living Residents With Dementia. The Gerontologist 2005;45:19-26.

3. Cuijpers P. Depressive disorders in caregivers of dementia patients: a systematic review. Aging \&3 Mental Health 2005;9:325-330.

4. de Vugt ME, Nicolson NA, Aalten P, Lousberg R, Jolle J, Verhey FRJ. Behavioral Problems in Dementia Patients and Salivary Cortisol Patterns in Caregivers. Journal of Neuropsychiatry \&5 Clinical Neurosciences 2005;17:201-207.

5. Aalten P, de Vugt ME, Jaspers N, Jolles J, Verhey FRJ. The course of neuropsychiatric symptoms in dementia. Part I: Findings from the two-year longitudinal Maasbed study. International Journal of Geriatric Psychiatry 2005;20:523-530.

6. Ballard CG, Margallo-Lana M, Fossey J, et al. A 1-year follow up study of behavioral and psychological symptoms in dementia among people in care environments. Journal of Clinical Psychiatry 2001;62:631-636.

7. Wetzels RB, Zuidema SU, de Jonghe JFM, Verhey FRJ, Koopmans RTCM. Course of neuropsychiatric symptoms in residents with dementia in nursing homes over 2-year period. The American Journal of Geriatric Psychiatry 2010;18:1054-1065.

8. Leon J, Moyer D. Potential cost savings in residential care for Alzheimer's disease patients. The Gerontologist 1999;39:440-449.

9. Reed J, Cantley C, Clarke CL, Stanley D. Services for younger people with dementia: Problems with differentiating needs on the basis of age. Dementia: The International Journal of Social Research and Practice 2002;1:95-112.

10. Rosness TA, Barca ML, Engedal K. Occurrence of depression and its correlates in early onset dementia patients. International Journal of Geriatric Psychiatry 2010;25:704-711.

11. Toyota Y, Ikeda M, Shinagawa S, et al. Comparison of behavioral and psychological symptoms in early-onset and late-onset Alzheimer's disease. International Journal of Geriatric Psychiatry 2007;22:896-901.

12. Hori K, Oda T, Asaoka T, et al. First episodes of behavioral symptoms in Alzheimer's disease patients at age 90 and over, and early-onset Alzheimer's disease: comparison with senile dementia of Alzheimer's type. Psychiatry and Clinical Neurosciences 2005;59:730-735.

13. Lawlor BA, Ryan TM, Schmeidler J, Mohs RC, Davis KL. Clinical symptoms associated with age at onset in Alzheimer's disease. The American Journal of Psychiatry 1994;151:1646-1649.

14. Rubin EH, Kinscherf DA, Morris JC. Psychopathology in younger versus older persons with very mild and mild dementia of the Alzheimer type. The American Journal of Psychiatry 1993;150:639-642. 
15. van Vliet D, Bakker C, Koopmans RT, Vernooij-Dassen MJ, Verhey FR, de Vugt ME. Research protocol of the NeedYD-study (Needs in Young onset Dementia): a prospective cohort study on the needs and course of early onset dementia. BMC Geriatrics 2010;10:13.

16. American Psychiatric Association. Diagnostic and Statistical manual of mental Disorders- Text revision: DSM-IV-TR. American Psychiatric Association 2000.

17. CBO: Guideline diagnosis and pharmacological treatment of dementia. 2005. Available at: http://www.cbo.nl/Downloads/387/rldement2005.pdf. Accessed: March 3, 2012.

18. McKhann G, Drachman D, Folstein M, Katzman R, Price D, Stadlan EM. Clinical diagnosis of Alzheimer's disease: report of the NINCDS-ADRDA Work Group under the auspices of Department of Health and Human Services Task Force on Alzheimer's Disease. Neurology 1984;34:939-944.

19. McKeith IG. Consensus guidelines for the clinical and pathologic diagnosis of dementia with Lewy bodies (DLB): report of the Consortium on DLB International Workshop. Journal of Alzheimer's Disease 2006;9:417-423.

20. Neary D, Snowden JS, Gustafson L, et al. Frontotemporal lobar degeneration: a consensus on clinical diagnostic criteria. Neurology 1998;51:1546-1554.

21. Mesulam MM, Grossman M, Hillis A, Kertesz A, Weintraub S. The core and halo of primary progressive aphasia and semantic dementia. Annals of Neurology 2003;54 Suppl 5:S11-14.

22. Erkinjuntti T. Clinical criteria for vascular dementia: The NINDS-AIREN criteria. Dementia 1994;5:189-192.

23. de Vugt ME, Jolles J, van Osch L, et al. Cognitive functioning in spousal caregivers of dementia patients: findings from the prospective MAASBED study. Age and Ageing 2006;35:160-166.

24. Cummings JL, Mega M, Gray K, Rosenberg-Thompson S. The Neuropsychiatric Inventory: Comprehensive assessment of psychopathology in dementia. Neurology 1994;44:2308-2314.

25. Kat MG, de Jonghe JFM, Aalten P, Kalisvaart CJ, Dres RM, Verhey FRJ. Neuropsychiatrische symptomen bij dementie: Psychometrische aspecten van de Nerlandse Neuropsychiatric Inventory (NPI). Tijdschrift voor Gerontologie en Geriatrie 2002;33:150-155.

26. Reisberg B, Ferris SH, de Leon MJ, Crook T. The Global Deterioration Scale for assessment of primary degenerative dementia. American Journal of Psychiatry 1982;139:1136-1139.

27. Folstein MF, Folstein SE, McHugh PR. Mini-mental state: A practical method for grading the cognitive state of patients for the clinician. Journal of Psychiatric Research 1975;12:189-198.

28. Steinberg M, Shao H, Zandi P, et al. Point and 5-year period prevalence of neuropsychiatric symptoms in dementia: The Cache County Study. International Journal of Geriatric Psychiatry 2008;23:170-177.

29. Steinberg M, Tschanz JT, Corcoran C, et al. The persistence of neuropsychiatric symptoms in dementia: the Cache County Study. 2004;19:19-26. 
30. Haupt M, Kurz A. Predictors of nursing home placement in patients with Alzheimer's disease. 1993;8:741-746.

31. Ferran J, Ferran J, Wilson K, Doran M, Ghadiali E. The early onset dementias: A study of clinical characteristics and service use. International Journal of Geriatric Psychiatry 1996;11:863-869.

32. Harvey RJ, Rossor MN, Skelton-Robinson M, Garralda E. Young onset dementia: epidemiology, clinical symptoms, family burden, support and outcome. Imperial college of science, technology and medicine Dementia research group 1998.

33. de Vugt ME, Riedijk SR, Aalten P, Tibben A, van Swieten JC, Verhey FR. Impact of behavioural problems on spousal caregivers: a comparison between Alzheimer's disease and frontotemporal dementia. Dementia and Geriatric Cognitive Disorders 2006;22:35-41.

34. de Vugt ME, Stevens F, Aalten P, et al. Behavioural disturbances in dementia patients and quality of the marital relationship. International Journal of Geriatric Psychiatry 2003;18:149-154.

35. Payne JL, Sheppard J-ME, Steinberg M, et al. Incidence, prevalence, and outcomes of depression in residents of a long-term care facility with dementia. International Journal of Geriatric Psychiatry 2002;17:247-253.

36. van Vliet D, de Vugt ME, Khler S, et al. Awareness and its association with affective symptoms in young-onset and late-onset Alzheimer's disease: A prospective study. Submitted for publication 2012.

37. Koedam EL, Lauffer V, van der Vlies AE, van der Flier WM, Scheltens P, Pijnenburg YA. Early-versus late-onset Alzheimer's disease: more than age alone. Journal of Alzheimer's Disease 2010;19:1401-1408.

38. van der Flier WM, Pijnenburg YAL, Fox NC, Scheltens P. Early-onset versus lateonset Alzheimer's disease: The case of the missing APOE E4 allele. The Lancet Neurology 2010;10:280-288.

39. van der Flier WM, Staekenborg S, Pijnenburg YA, et al. Apolipoprotein E genotype influences presence and severity of delusions and aggressive behavior in Alzheimer disease. Dementia and Geriatric Cognitive Disorders 2007;23:42-46.

40. Savva GM, Zaccai J, Matthews FE, Davidson JE, McKeith I, Brayne C. Prevalence, correlates and course of behavioural and psychological symptoms of dementia in the population. British Journal of Psychiatry 2009;194:212-219.

41. Drzezga A, Grimmer T, Henriksen G, et al. Effect of APOE genotype on amyloid plaque load and gray matter volume in Alzheimer disease. Neurology 2009;72:14871494.

42. Cohen-Mansfield J. Nonpharmacologic Interventions for Psychotic Symptoms in Dementia. Journal of Geriatric Psychiatry and Neurology 2003;16:219-224.

43. Brown EL, Raue P, Halpert KD, Adams S, Titler MG. Evidence-Based Guideline Detection of Depression in Older Adults with Dementia. Journal of Gerontological Nursing 2009;35:11-15.

44. Richards M, Brayne C. What do we mean by Alzheimer's disease? British Medical Journal 2010;341:c4670. 
45. Kohler S, Verhey FR. Cognitive deficits in late-life depression. Tijdschrift voor Psychiatrie 2011;53:601-607.

46. Brunoni AR, Bensenor IM, Alves TC. Therapeutic interventions for vascular depression: a systematic review. Revista Brasileira de Psiquiatria 2011;33:400-409.

47. Olin JT, Schneider LS, Katz IR, et al. Provisional diagnostic criteria for depression of Alzheimer disease. The American Journal of Geriatric Psychiatry 2002;10:125128.

48. Engedal K, Barca ML, Laks J, Selbaek G. Depression in Alzheimer's disease: specificity of depressive symptoms using three different clinical criteria. International Journal of Geriatric Psychiatry 2011;26:944-951.

49. Starkstein SE, Ingram L, Garau ML, Mizrahi R. On the overlap between apathy and depression in dementia. Journal of Neurology, Neurosurgery $\&$ Psychiatry 2005;76:1070-1074.

50. Starkstein SE, Brockman S, Bruce D, Petracca G. Anosognosia is a significant predictor of apathy in Alzheimer's disease. The Journal of Neuropsychiatry and Clinical Neurosciences 2010;22:378-383.

51. Starkstein SE, Jorge R, Mizrahi R, Robinson RG. A prospective longitudinal study of apathy in Alzheimer's disease. Journal of Neurology, Neurosurgery, and Psychiatry 2006;77:8-11.

52. Cummings JL, Mackell J, Kaufer D. Behavioral effects of current Alzheimer's disease treatments: A descriptive review. Alzheimer's \& Dementia 2008;4:49-60.

53. Wynn ZJ, Cummings JL. Cholinesterase inhibitor therapies and neuropsychiatric manifestations of Alzheimer's disease. Dementia and Geriatric Cognitive Disorders 2003;17:100-108.

54. Aalten P, Verhey FR, Boziki M, et al. Consistency of neuropsychiatric syndromes across dementias: results from the European Alzheimer Disease Consortium. Part II. Dementia and Geriatric Cognitive Disorders 2008;25:1-8.

55. Bourgeois J, Elseviers MM, Azermai M, Van Bortel L, Petrovic M, Vander Stichele RR. Benzodiazepine use in Belgian nursing homes: a closer look into indications and dosages. European Journal of Clinical Pharmacology 2011.

56. Rosenberg PB, Mielke MM, Han D, et al. The association of psychotropic medication use with the cognitive, functional, and neuropsychiatric trajectory of Alzheimer's disease. International Journal of Geriatric Psychiatry 2012.

57. Arai A, Matsumoto T, Ikeda M, Arai Y. Do family caregivers perceive more difficulty when they look after patients with early onset dementia compared to those with late onset dementia? International Journal of Geriatric Psychiatry 2007;22:1255-1261. 


\section{Chapter 7}

\section{Awareness and its association with affective symptoms in young-onset and late-onset Alzheimer's disease}

Based on: van Vliet D, de Vugt ME, Khöler S, Aalten P, Bakker C, Pijnenburg YAL, Vernooij-Dassen MJFJ, Koopmans RTCM, Verhey FRJ (2012). Awareness and its association with affective symptoms in young-onset and late-onset Alzheimer's disease: A prospective study. Alzheimer's Disease \&6 Associated Disorders. In press. 


\begin{abstract}
Background It is unknown whether there are differences between young-onset dementia and late-onset dementia in awareness levels and whether awareness is differentially associated with affective symptoms in both groups. The present study assesses possible differences between young-onset (YO-AD) and late-onset Alzheimer's disease (LO-AD) in awareness levels and the association between awareness and affective symptoms.
\end{abstract}

Methods This study included 142 YO-AD and 126 LO-AD patients and their caregivers from two prospective studies. The participants were assessed three times during one year. Awareness was assessed using the Guidelines for the Rating of Awareness Deficits, and affective symptoms were assessed using the anxiety and depression items of the Neuropsychiatric Inventory. Population-averaged logistic regressions were used to analyze awareness and its association with affective symptoms.

Results The odds for impaired awareness in LO-AD were more than double the odds in YO-AD. Intact awareness was associated with depressive symptoms but not with anxiety. This effect was more pronounced in YO-AD compared with LO-AD at baseline. High awareness at baseline did not predict incident affective symptoms.

Conclusions Caregivers and clinicians should be prepared for affective symptoms in YO-AD patients with high awareness. The higher awareness in the YO-AD group also has potential positive implications for this group. 


\subsection{Introduction}

Awareness, which can be defined as the capability of an individual to accurately evaluate and report about his abilities and limitations, is often impaired in dementia ${ }^{1}$ and has important associations with behavioral and psychological symptoms $^{2,3}$. The investigation of awareness is hampered due to varying terminology and conceptualizations (e.g., lack of insight, denial of illness or anosognosia) and a lack of standardized assessment methods ${ }^{4,5}$. Awareness is a complex construct because it can be impaired differently in several domains (e.g., cognitive, functional $)^{6}$. Furthermore, a dissociation exists between implicit and explicit awareness ${ }^{7}$. Research into this phenomenon has mainly focused on dementia in old age and has shown that high levels of awareness are associated with dysthymia and anxiety but not with major depression ${ }^{4,8}$. Gaining insight into these mechanisms in young-onset dementia (YOD) would provide important information to determine which patients are at risk for developing affective symptoms and to adequately support YOD patients and their caregivers.

Anecdotal evidence suggests that YOD patients have more insight into their condition than do late-onset dementia (LOD) patients ${ }^{9,}{ }^{10}$. Several empirical studies have found younger age (at symptom onset) to be associated with increased deficit awareness, but no studies have specifically focused on YOD ${ }^{8,11,12}$. The idea that people with YOD have higher levels of awareness is plausible. In addition to neurological factors, the degree of awareness is dependent on psychosocial aspects, such as defense mechanisms, social factors and coping abilities ${ }^{7}$. For instance, it has been hypothesized that dementia patients with high cognitive reserve are more likely to participate in cognitively challenging activities and thereby increase their awareness of deficits ${ }^{2}$. Similarly, awareness levels in YOD patients may be increased because these patients are confronted with more challenging activities, a more demanding environment and losses in roles and status related to their life phase. They fulfill active social roles (e.g., worker, main income provider, parent, road user) when the first symptoms emerge. These losses in role and status are higher than in LOD and have a major impact on YOD patients ${ }^{13}$. Therefore, awareness may lead to a higher risk of emotional reactions and decreases quality of life for YOD more than for LOD.

The present study aims to assess whether there are differences between the level of awareness in YOD and LOD and to test its association with affective symptoms (anxiety and depression). It is hypothesized that 1) YOD patients show higher levels of awareness than LOD patients, 2) intact awareness is predictive of developing depression and anxiety and 3) the association between awareness and affective symptoms is stronger in YOD patients than in LOD patients. These findings may provide important information for clinicians to determine which patients are at risk and may contribute to the knowledge base on the etiology of awareness and affective symptoms in dementia. 


\subsection{Methods}

\section{Subjects}

This study is part of a Dutch prospective cohort study, the NeedYD study (Needs in Young-onset Dementia), which has been described in detail elsewhere ${ }^{14}$. Beginning in 2007, 215 participants with YOD with symptom onset before the age of 65 were recruited. Assessments with patients and their primary caregivers were undertaken every six months for two years. Patients were recruited from YODspecialized day care facilities $(\mathrm{n}=115)$, consecutive referrals from University Medical Centers in Maastricht and Amsterdam $(n=56)$, regional hospitals $(n=10)$, regional community mental health services $(n=20)$ or they applied individually to participate $(\mathrm{n}=14)$. Inclusion criteria were symptom onset before the age of 65 , a dementia diagnosis and availability of an informant who had regular contact with the patient (once per week minimum). Dementia diagnoses were made on the basis of clinical, neuropsychological and brain imaging findings according to the criteria from the $4^{\text {th }}$ edition of the Diagnostic and Statistical Manual of Mental Disorders, text revision $(2000)^{15}$ and the Dutch consensus guidelines ${ }^{16}$, which use internationally accepted criteria for diagnosing dementia subtypes ${ }^{17-21}$. Clinical diagnoses were checked against clinical files for every patient and against medical hospital records, if available. Exclusion criteria were (1) lack of informed consent of the participant, (2) institutionalization and (3) dementia caused by HIV, traumatic brain injury, Down's syndrome, Huntington's chorea or alcohol-related dementia.

A comparison group was selected from a 2-year follow-up cohort study of 199 LOD patients, the MAASBED study (MAAstricht Study of BEhaviour in Dementia $)^{22}$, which used essentially the same design, assessment instruments and diagnostic criteria. In the MAASBED -study, patients were consecutively enrolled from the memory clinic of the Maastricht University Medical Center or the Community Mental Health Care Team (RIAGG) in Maastricht, irrespective of diagnostic subtype. The MAASBED cohort also included 26 YOD patients and these patients were added to the YOD group. For the current study, patients were included if they had Alzheimer's disease (AD), as diagnosed according to the NINCDS-ADRDA criteria ${ }^{17}$ and if the primary caregiver was an informal caregiver. This recruitment resulted in 142 patients with young-onset AD (YO-AD) and 126 patients with late-onset AD (LO-AD). Data from baseline, 6-month and 12-month follow-up assessments were analyzed for the present study. The study protocol was approved by the Medical Ethics Committee of the University Medical Center Maastricht. Written informed consent of the subjects or their legal representatives was obtained prior to the investigation. 


\section{Assessment of awareness}

Awareness was assessed with the Guidelines for the Rating of Awareness Deficits (GRAD), which defines impaired awareness as the absence of explicit knowledge or recognition of one's own cognitive deficits ${ }^{11}$. The GRAD is a reliable (kappa value of 0.70) semi-structured interview in which the degree of awareness is assessed by comparing the patient's and the caregiver's histories of the patient's functioning and complaints ${ }^{11,23}$. Awareness is rated on a four-point scale (4 (intact), 3 (mildly disturbed), 2 (moderately disturbed), 1 (awareness absent)).

\section{Assessment of affective symptoms}

Symptoms of depression and anxiety were assessed by the Neuropsychiatric Inventory $(\mathrm{NPI})^{24}$. The NPI is a structured interview with the primary caregiver and the most widely used instrument to determine neuropsychiatric problems in dementia, assessing ten neuropsychiatric and two neuro-vegetative symptoms. The NPI begins with screening questions to determine whether behavioral changes are present. The frequency and severity of each symptom are rated on a five- and four-point Likert scale, respectively. These scores are multiplied to obtain total item scores ranging from $0-12$. The reliability and validity of the Dutch version of the NPI have been demonstrated ${ }^{25}$. Because the NPI scores were not normally distributed, they were dichotomized. To obtain equal group sizes for patients with absent and present affective symptoms, the cut-off was set at NPI $>0$ instead of NPI $>3$. Prevalent depression and anxiety were defined as an NPI item score $>$ 0 . Incident depression and anxiety were defined as emerging symptoms with NPI item scores $>0$ and the absence of these symptoms at prior assessments.

\section{Assessment of covariates}

Age, sex, level of education (ranging from (1) primary school to (8) university degree), disease duration (years) and patients' medication use were obtained from the primary caregivers. The Global Deterioration Scale (GDS), which is a valid and widely used seven-point rating scale (1-7) ranging from 'no impairment' (1) to 'very severe cognitive impairment' (7), was used to rate dementia severity. For the present study, GDS scores were divided into three categories: mild (scores 3, 4 ), moderate (score 5) and severe (scores 6, 7).

\section{Statistical analysis}

Differences between the YO-AD and LO-AD groups in baseline characteristics were assessed with t-tests for continuous variables and chi-square tests for categorical variables. If the assumptions for parametric testing were not met, Mann-Whitney $\mathrm{U}$ tests were conducted instead.

For the main analyses, data were pooled across assessments to increase power. We then fitted population-averaged logistic regression models using generalized 
estimating equations (GEE) for the binomial family with a logit-link function. This type of analysis accounts for correlations among observations due to repeated measurements (i.e., observations in time nested within individuals).

To assess whether groups had differential associations with impaired awareness across the entire observational period (hypothesis 1), analyses were conducted with awareness as a dependent variable and group as an independent variable. To test for differences between groups in changes in awareness over time, an interaction term (group X time) was added to this model. Analyses were further adjusted for gender, education, GDS score at each assessment and time (baseline, 6-month FU and 12-month FU). For this analysis, the GRAD scores were dichotomized into impaired (GRAD scores 1\&2) versus intact awareness (GRAD scores $3 \& 4$ ).

Next, we investigated whether awareness was related to depression and anxiety (hypothesis 2), both cross-sectionally (prevalent symptoms) and longitudinally (incident symptoms). Again, pooled population-averaged logistic regression analyses were run with prevalent and incident depression and anxiety as dependent variables and GRAD and group as independent variables. A term for the interaction between group and awareness was added to assess whether the association between awareness and affective symptoms was similar across groups (hypothesis 3). These analyses were again corrected for gender, education, GDS score at each assessment and time (baseline, 6-month FU, and 12-month FU). Post-hoc analyses were performed to identify any group-by-awareness interactions at any of the three assessments. Separate logistic regression analyses were performed that controlled for education, gender and dementia severity, with prevalent depression and anxiety as dependent variables and group, GRAD and the GRAD by Group interaction as independent variables. An alpha level of .05 was considered significant, and all tests were two-sided. Analyses were performed in Stata 11.1 for OS $\mathrm{X}$ (StataCorp, Texas).

\section{Missing data}

GEE assumes that missing data are missing completely at random, but in observational studies, observations are generally missing at random. Furthermore, missing covariates present a problem when using GEE because inconsistent GEE estimates may be produced by discarding the incomplete cases. A common solution to these problems is to impute missing observations. Missing data were therefore augmented using multiple imputations (MI). Twenty complete versions of the dataset were constructed provided that i) the reason for missing data was other than death, and ii) the variable was observed in a minimum of $60 \%$ of cases using Stata's MI IMPUTE command. Excluding patients who had died $(\mathrm{N}=18)$, a total of $20.1 \%$ of the cases had either item- or unit-level missing data in the NPI, GRAD or GDS and were therefore imputed twenty times. The imputation model consisted of factors potentially associated with these variables, factors thought to predict missingness in general and all variables used in the intended statistical 
Table 7.1: Baseline characteristics of patients with young-onset (YO-AD) and late-onset (LO-AD) Alzheimer's disease.

\begin{tabular}{|c|c|c|c|c|}
\hline & $\begin{array}{l}\text { YO-AD } \\
(\mathrm{n}=142)\end{array}$ & $\begin{array}{r}\text { LO-AD } \\
(\mathrm{N}=126)\end{array}$ & Test value & $p$-value \\
\hline Age, mean (SD) & $61.6(4.8)$ & $79.1(6.1)$ & $U=17789.5$ & $<.001$ \\
\hline Disease duration in years, mean (SD) & $6.5(3.6)$ & $3.0(2.2)$ & $U=3084.0$ & $<.001$ \\
\hline Female, n $(\%)$ & $73(51.4)$ & $84(66.7)$ & $\chi^{2}(\mathrm{df}=1)=6.4$ & .011 \\
\hline \multicolumn{5}{|l|}{ Education, n (\%) } \\
\hline Low $(1-2)$ & $61(43.0)$ & $88(69.8)$ & $\chi^{2}(\mathrm{df}=2)=22.3$ & $<.001$ \\
\hline Medium (3-5) & $46(32.4)$ & $28(22.2)$ & & \\
\hline High (6-8) & $35(24.6)$ & $10(7.9)$ & & \\
\hline \multicolumn{5}{|l|}{ GDS score, n (\%) } \\
\hline Mild (2-4) & $68(49.6)$ & $97(77.0)$ & $\chi^{2}(\mathrm{df}=2)=34.1$ & $<.001$ \\
\hline Moderate (5) & $42(30.7)$ & $29(23.0)$ & & \\
\hline Severe $(6,7)$ & $\begin{array}{r}27(19.7) \\
(\mathrm{n}=137)\end{array}$ & $0(0.0)$ & & \\
\hline \multicolumn{5}{|l|}{ Psychoactive medication, n (\%) } \\
\hline Antipsychotic & $18(12.7)$ & $18(14.3)$ & $\chi^{2}(\mathrm{df}=1)=0.1$ & .700 \\
\hline Antidepressant & $46(32.4)$ & $28(22.2)$ & $\chi^{2}(\mathrm{df}=1)=3.5$ & .063 \\
\hline Benzodiazepines & $6(4.2)$ & $36(28.6)$ & $\chi^{2}(\mathrm{df}=1)=29.9$ & $<.001$ \\
\hline Antidementia & $90(63.4)$ & $7(5.6)$ & $\chi^{2}(\mathrm{df}=1)=96.7$ & $<.001$ \\
\hline Any of the above & $103(72.5)$ & $62(49.2)$ & $\chi^{2}(\mathrm{df}=1)=15.4$ & $<.001$ \\
\hline \multicolumn{5}{|l|}{ Awareness (GRAD), n (\%) } \\
\hline Severely disturbed & $21(15.6)$ & $26(20.6)$ & $\chi^{2}(\mathrm{df}=1)=4.6$ & .201 \\
\hline Moderately disturbed & $34(25.2)$ & $41(32.5)$ & & \\
\hline Mildly disturbed & $52(38.5)$ & $42(33.3)$ & & \\
\hline Intact & $28(20.7)$ & $17(13.5)$ & & \\
\hline \multicolumn{5}{|l|}{ NPI depression } \\
\hline $\mathrm{n}(\%)$ & $79(55.6)$ & $3(57.9)$ & $\chi^{2}(\mathrm{df}=1)=0.1$ & .753 \\
\hline mean $(\mathrm{sd})$ & $2.0(2.8)$ & $3.5(3.5)$ & $U=7599.0$ & .033 \\
\hline \multicolumn{5}{|l|}{ NPI anxiety } \\
\hline $\mathrm{n}(\%)$ & $63(44.4)$ & $54(42.9)$ & $\chi^{2}(\mathrm{df}=1)=0.1$ & .725 \\
\hline mean (sd) & $1.7(2.8)$ & $2.2(3.6)$ & $U=8657.5$ & .775 \\
\hline
\end{tabular}

analyses, including relevant interaction terms. The statistical analyses were run using Stata's MI ESTIMATE prefix.

\section{$7.3 \quad$ Results}

\section{Sample characteristics}

A total of 268 patients were included in the study. At the six-month follow-up, 10 participants refused (4 YO-AD, 6 LO-AD), 18 participants were lost to follow-up (2 YO-AD, 16 LO-AD) and 9 patients had died (1 YO-AD, 8 LO-AD). After one 
year, 10 participants refused (4 YO-AD, 6 LO-AD) and 9 had died (3 YO-AD, 6 LO-AD). Data for patients who dropped out of the study for reasons other than death were imputed as described above.

Table 7.1 shows the baseline characteristics of the two groups. The YO-AD group consisted of more males, the educational level was higher overall and the dementia stage (GDS) was more severe than in the LO-AD group. The mean disease duration was longer in the YO-AD group. Fewer YOD patients used benzodiazepines, but the use of antidementia medication was more frequent in the YO-AD group than in the LO-AD group. Awareness scores at baseline did not significantly differ between groups. The number of patients with depressive and anxiety symptoms and the mean anxiety score did not differ between groups, but the number of patients with depressive symptoms at baseline was higher in the LO-AD group.

\section{Differences in awareness between YO-AD and LO-AD over the one year-period}

Figure 7.1 shows the distribution of awareness scores for YO-AD and LO-AD across assessment waves. Pooled across assessments, the LO-AD group was more likely to have impaired awareness compared with the YO-AD group $(\mathrm{OR}=2.40$, $95 \% \mathrm{CI}=1.47-3.90, \mathrm{p}=<.001)$. This effect was not moderated by time $\left(\chi^{2}\right.$ for interaction $=0.90, \mathrm{df}=2, \mathrm{p}=0.638)$. Over the one-year period, females appeared to have higher odds for impaired awareness $(\mathrm{OR}=1.63,95 \% \mathrm{CI}=1.04-2.56, \mathrm{p}=$ $.035)$ and awareness decreased with increasing dementia severity (moderate versus mild: $\mathrm{OR}=1.99,95 \% \mathrm{CI}=1.39-2.85, \mathrm{p}<.001$; severe versus mild: $\mathrm{OR}=8.05$, $95 \% \mathrm{CI}=4.11-15.77, \mathrm{p}<.001)$.

\section{Awareness and prevalent affective symptoms over the one year-period}

Analyses pooled across assessments with prevalent depression and anxiety as dependent variables showed that the level of awareness was inversely associated with depression, meaning that patients with intact $(\mathrm{OR}=1.91,95 \% \mathrm{CI}=1.05-3.48$, $\mathrm{p}=.034)$ and mildly disturbed $(\mathrm{OR}=2.15,95 \% \mathrm{CI}=1.30-3.54, \mathrm{p}=.003)$ awareness had greater odds of having depression than patients with severely disturbed awareness over the one-year period (Table 7.2). No significant overall groupby-awareness interaction was found ( $\chi^{2}$ for interaction $=3.98, \mathrm{df}=3, \mathrm{p}=.263$ ), indicating that the strength of the association between awareness and depressive symptoms was similar between the groups over the study period. The pooled analyses over the one-year period did not show a relationship between awareness and anxiety (Table 7.2) or a group-by-awareness interaction for anxiety $\left(\chi^{2}\right.$ for interaction $=0.44, \mathrm{df}=3, \mathrm{p}=.932)$. 

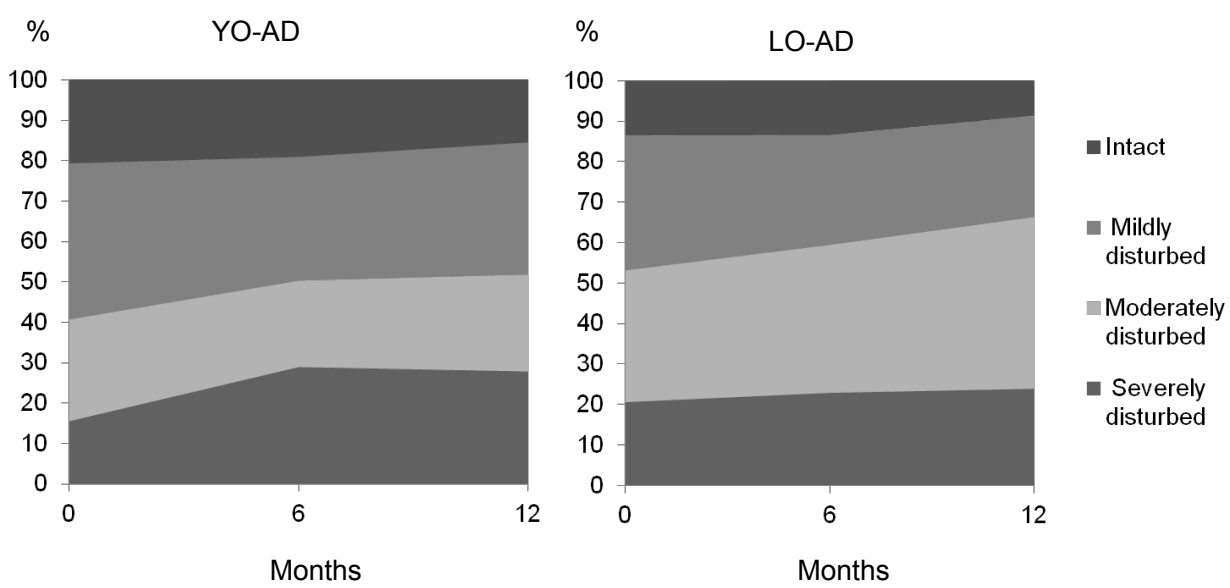

Figure 7.1: Distribution of non-imputed awareness scores over time for YO-AD and LO-AD.

Separate post-hoc analyses for each assessment Because one of our main research aims was to assess whether awareness and affective symptoms were differentially related in YO-AD and LO-AD, we explored whether group-by-awareness interaction effects were present at any of the three assessment waves. One interaction effect was found for depression at baseline $\left(\chi^{2}\right.$ for interaction $=5.13, \mathrm{df}=$ $1, \mathrm{p}=.024$ ), indicating that, relative to patients with impaired awareness, YOAD patients with intact awareness had a higher risk of depressive symptoms than LO-AD patients with intact awareness. Results stratified by group showed a clear relationship of increasing odds for depressive symptoms with increasing awareness in the YO-AD group (moderate versus severe: $\mathrm{OR}=2.11,95 \% \mathrm{CI}=0.62-7.23, \mathrm{p}=$ .233 ; mild versus severe: $\mathrm{OR}=4.08,95 \% \mathrm{CI}=1.11-14.94, \mathrm{p}=0.34$; intact versus severe: $\mathrm{OR}=5.73,95 \% \mathrm{CI}=1.31-25.11, \mathrm{p}=.021)$. In contrast, no significant associations were found in the LO-AD group.

\section{Awareness and incident affective symptoms over the one year-period}

To investigate contributions of the baseline level of awareness and group on incident depression and anxiety over the one-year period, population-averaged logistic regressions were performed, adjusted for education, gender, disease severity and follow-up assessment (to pool effects across assessments). No significant effects were observed for baseline level of awareness or group on the odds of incident depression or anxiety pooled over follow-up (Table 7.2). Interaction effects of group-by-awareness were also not statistically significant for depression $\left(\chi^{2}\right.$ for interaction $=0.40, \mathrm{df}=3, \mathrm{p}=.941$ ) or anxiety $\left(\chi^{2}\right.$ for interaction $\left.=4.16, \mathrm{df}=3, \mathrm{p}=.245\right)$. 
Table 7.2: The effect of awareness and group on prevalent and incident depression and anxiety.

\begin{tabular}{|c|c|c|c|c|c|c|c|c|}
\hline & \multicolumn{4}{|c|}{ Prevalence } & \multicolumn{4}{|c|}{ Incidence } \\
\hline & $\mathbf{N}(\%)$ & OR & $95 \% \mathrm{CI}$ & $p$ & $\mathbf{N}(\%)$ & OR & $95 \% \mathrm{CI}$ & $p$ \\
\hline \multicolumn{9}{|l|}{ Main effects depression } \\
\hline Group: YO-AD a & $213(53.0)$ & 1.00 & & & $36(15.3)$ & 1.00 & & \\
\hline LO-AD & $171(54.5)$ & 1.44 & $0.94-2.23$ & .097 & $20(11.6)$ & 2.12 & $0.99-4.54$ & .053 \\
\hline \multicolumn{9}{|c|}{ Baseline level of awareness } \\
\hline Severely disturbed ${ }^{a}$ & $78(48.1)$ & 1.00 & & & $8(12.7)$ & 1.00 & & \\
\hline Moderately disturbed & $101(49.0)$ & 1.32 & $0.84-2.06$ & .232 & $19(16.0)$ & 1.41 & $0.53-3.71$ & .488 \\
\hline Mildly disturbed & $137(31.7)$ & 2.15 & $1.30-3.54$ & .003 & $14(9.8)$ & 1.46 & $0.47-4.59$ & .516 \\
\hline Intact & $59(53.2)$ & 1.91 & $1.05-3.48$ & .034 & $12(16.7)$ & 1.96 & $0.61-6.31$ & .261 \\
\hline \multicolumn{9}{|l|}{ Main effects anxiety } \\
\hline Group: YO-AD ${ }^{\text {a }}$ & $156(38.9)$ & 1.00 & & & $44(18.6)$ & 1.00 & & \\
\hline LO-AD & $118(37.6)$ & 1.17 & $0.61-1.77$ & .462 & $28(16.2)$ & 1.29 & $0.67-2.52$ & .446 \\
\hline \multicolumn{9}{|l|}{ Baseline level of awareness } \\
\hline Severely disturbed ${ }^{a}$ & $54(33.5)$ & 1.00 & & & $9(14.3)$ & 1.00 & & \\
\hline Moderately disturbed & $76(36.9)$ & 1.1 & $0.68-1.77$ & .698 & $15(12.6)$ & 1.38 & $0.53-3.63$ & .514 \\
\hline Mildly disturbed & $91(41.0)$ & 1.31 & $0.78-2.22$ & .306 & $26(18.2)$ & 1.54 & $0.56-4.24$ & .405 \\
\hline Intact & $47(42.3)$ & 1.29 & $0.70-2.36$ & .410 & $19(26.4)$ & 2.01 & $0.65-6.20$ & .226 \\
\hline
\end{tabular}

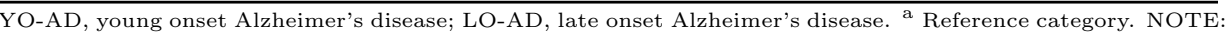
descriptives are shown for non-imputed data.

\subsection{Discussion}

The present study is the first to examine awareness and its association with affective symptoms in YO-AD. The findings support our hypothesis that patients with YO-AD have higher levels of awareness than LO-AD patients. The odds of impaired awareness were more than two times higher in the latter group. Furthermore, our other hypotheses were partly confirmed: intact awareness was associated with depressive symptoms, and this effect was more pronounced in YO-AD compared with LO-AD at baseline. In contrast, we found no support that high awareness at baseline predicted incident affective symptoms.

\section{Awareness and age at onset}

The current findings are in line with previous studies that have found younger age or younger age at onset to be related to higher awareness ${ }^{8,11,12}$. However, other studies have failed to find such a relationship ${ }^{6,26-29}$, which may be due to their smaller sample sizes or differences in the methods used. Overall, weaker correlations have been found between awareness and age at onset than between awareness and age. However, none of these studies specifically focused on YOD. Hence, the present study is the first to show that patients with YO-AD have, on average, less impaired awareness than patients with LO-AD. Future studies are needed to confirm this finding and to explore its potential bio-psychosocial origin. For instance, the different psychosocial context in which YO-AD emerges might 
play an important role, as mentioned previously. The possible higher environmental demands faced by younger people may make them more aware of their limitations. Another explanation might relate to the underlying neuropathology. Some YO-AD patients show an atypical non-memory phenotype with a pattern of more severe atrophy in posterior regions of the brain and less severe atrophy of the medial temporal lobe compared to LO-AD patients ${ }^{30}$. This structure has been linked to awareness ${ }^{31}$. In addition, YO-AD patients commonly do not carry the $\mathrm{APOE} \varepsilon 4$ allele, and the absence of this allele has been associated with lower $\beta$ amyloid deposition in frontal cortical areas ${ }^{32}$. Therefore, the frontal lobes, which are known to be involved in awareness ${ }^{33}$, may be less severely damaged in YO-AD. Furthermore, a partial explanation for the higher awareness in YO-AD may be the atypical cognitive profile with relatively preserved memory capacity in some YO-AD patients. These patients are less likely to 'forget that they forget'. However, the link with memory deficits is unlikely to sufficiently explain impaired awareness, since patients with mild dementia can display impaired awareness and patients with severely disturbed memory retention and consolidation still can have intact awareness ${ }^{3,31}$.

\section{Awareness and prevalent affective symptoms}

The present study found evidence that higher levels of awareness were associated with a higher risk of depressive symptoms. This finding is in line with our previous MAASBED report on $\mathrm{LOD}^{8}$ and with other studies ${ }^{26,34}$. In contrast, a number of studies have not found this relationship ${ }^{11,27,29}$. These inconsistencies have been attributed to the use of varying criteria for determining depression; for example, studies using dysthymia as an outcome showed positive results, whereas studies of major depression were largely negative ${ }^{4}$. This difference has been confirmed in studies directly comparing AD patients with dysthymia with patients with major depression $^{28,35}$. These findings have led to the hypothesis that dysthymia in dementia is an emotional situational response to cognitive decline, whereas major depression is caused by biological factors unrelated to awareness ${ }^{28}$. In the present study, we used a broad definition of depressive symptoms (NPI symptom scores ranging from 1 to 12). Hence, our results may be driven by the specific relationship between awareness levels and depressive symptoms in dysthymic patients only. In fact, a post-hoc analysis in which our cut-off for depressive symptoms was increased from an NPI score $>0$ to a score $>3$ did not show significant associations.

Unlike other studies ${ }^{8,11}$, we did not find an association between awareness and anxiety. This can be explained by the fact that previous studies have investigated this association only at single time points. For example, one study found awareness to be related to anxiety and depression at one out of 4 time points, suggesting that this relationship fluctuates over time or may be a spurious association ${ }^{8}$. It should be noted that depression and anxiety in dementia are highly co-morbid and have overlapping symptoms. In several studies, the association between awareness and anxiety disappeared after controlling for depressive symptoms ${ }^{36}$. 


\section{Awareness and incident affective symptoms}

In contrast to the cross-sectional relationships, no evidence was found that high awareness at baseline is a predictor for developing depressive symptoms or anxiety over time. This study is the first to assess incident affective symptoms; other studies have examined the worsening of prevalent symptoms ${ }^{8,37}$. Therefore, awareness may relate to the course of depressive symptoms without being a risk factor for it. However, a bi-directional relationship is most plausible because high awareness can also be the result of low mood (reverse causality), leading to subjective memory complaints ${ }^{38}$.

\section{Differences between YO-AD and LO-AD}

In exploratory analyses, we found that the relationship between awareness and depressive symptoms was more prominent in YO-AD compared with LO-AD. This finding was only observed at baseline and only when comparing severely impaired awareness with intact awareness (GRAD 1 versus 4). Therefore, it is questionable whether this is a true finding. The fact that this effect was only observed at baseline may suggest that YO-AD patients with high levels of awareness experience more difficulty coping with the dementia diagnosis and their progressive loss of cognitive abilities than LO-AD patients with comparable levels of awareness. Unfortunately, we were unable to study this phenomenon because only a portion of the YO-AD group consisted of consecutive referrals from memory clinics during an early phase of the disease. It may also be that a common biological factor in YO-AD underlies both the atypical brain atrophy and the clinical phenotype that includes higher awareness as well as depressive symptoms. Future studies examining the MRI correlates of awareness and their relation to behavioral symptoms would therefore be desirable.

\section{Strengths and limitations}

The current study is the first longitudinal study to compare the effect of an awareness deficit on affective symptoms between YOD and LOD in a large sample, using a reasonable follow-up period and commonly accepted methods of assessing levels of awareness and neuropsychiatric symptoms. The analyses in this study were not limited to study completers because complete case analyses (listwise deletion) is likely to lead to selection bias. Instead, we used commonly recommended but underutilized statistical techniques to handle missing data.

Nevertheless, some potential limitations must be considered. The YO-AD and LO-AD samples came from different studies with slightly different recruitment strategies. Although both studies sampled from memory clinics and institutes for community mental health and used essentially the same design, assessment measures and diagnostic criteria, the MAASBED study only included consecutive referrals after diagnosis, whereas the NeedYD study also included patients using 
specialized day care. This difference may have influenced the distributions of depressive symptoms and anxiety differentially.

In addition, disease duration was much longer in the YO-AD group. However, most studies found that disease duration was not related to level of awareness and the few studies finding a relationship showed that longer disease duration was related to lower levels of awareness ${ }^{4}$. Therefore, excluding this variable from the analyses may have resulted in an underestimation of the difference in awarenesslevels between YO-AD and LO-AD. We controlled for dementia severity instead, which is more strongly related to level of awareness ${ }^{4}, 33$. Furthermore, the GDS, which we used to measure dementia severity has been found to be a better predictor of impaired insight than duration of memory loss and the Mini Mental State Examination (MMSE), which is a measure of global cognitive impairment ${ }^{39}$.

Similarly, there were differences in medication use between the groups, which may have influenced the results. Antidementia medication use was strikingly low in $\mathrm{LO}-\mathrm{AD}$, probably because the $\mathrm{LO}-\mathrm{AD}$ patients were drawn from a study conducted 8 years earlier, when such medication was not widely available. In addition, more patients in the LO-AD group used benzodiazepines; therefore, the association between awareness and anxiety may have been underestimated in this group. Similarly, overall effects between awareness and depressive symptoms may be partly obscured by the use of antidepressant medication in both groups. The sample characteristics may also limit the generalizability of the findings to the entire YO-AD and LO-AD populations.

Furthermore, the use of a four-point rating scale to measure the level of awareness may not capture the complexity of this construct. The GRAD does not distinguish impaired awareness due to psychological mechanisms from pure anosognosia, and it only focuses on awareness of cognitive deficits. Awareness of cognitive, functional or behavioral deficits can be differentially affected in persons with dementia $^{6}$. Thus, a more meaningful relationship between awareness and affective symptoms may have been found if we had considered all of these aspects of awareness. Finally, it should be noted that the number of patients with incident depressive symptoms was small, and analyses might thus have been underpowered. The gradient effect of awareness on depressive symptoms and the skewed $95 \%$ confidence intervals suggest that we may have missed a true relationship. It must be noted that most patients ( $55 \%$ to $60 \%$ ) showed depressive symptoms at baseline and could not be considered at risk. Hence, larger samples may be needed.

\section{Implications}

Our findings indicate that caregivers and clinicians should be prepared for affective symptoms in YO-AD patients with high awareness. This information should guide clinicians involved in disclosing a diagnosis as well as subsequent counselling of YO-AD patients and their caregivers. The higher level of awareness in the YO$\mathrm{AD}$ group also has potential positive implications for this group. Awareness increases people's ability to recognize their limits and choose activities accordingly ${ }^{40}$. 
Therefore, YO-AD patients may be better able to participate in daily and social activities and to adjust to their level of functioning. Furthermore, treatment compliance is generally higher in patients with high awareness, which, in turn, leads to positive outcomes of cognitive rehabilitation in mild $\mathrm{AD}^{41}$. Further research is needed to test the potential benefits from such interventions for YO-AD patients. YO-AD patients may be better able to take a pro-active role in managing their condition and to make decisions about their care process and their future due to their higher awareness, and they should be given the opportunity to do so. 


\section{References}

1. Snow AL, Graham DP, Molinari VA, et al. Factors Affecting Deficit Awareness in Persons with Dementia. Dementia and Geriatric Cognitive Disorders 2005;20:133139.

2. Spitznagel MB, Tremont G, Brown LB, Gunstad J. Cognitive Reserve and the Relationship Between Depressive Symptoms and Awareness of Deficits in Dementia. The Journal of Neuropsychiatry and Clinical Neurosciences 2006;18:186-190.

3. Starkstein SE, Brockman S, Bruce D, Petracca G. Anosognosia is a significant predictor of apathy in Alzheimer's disease. The Journal of Neuropsychiatry and Clinical Neurosciences 2010;22:378-383.

4. Aalten P, Van Valen E, Clare L, Kenny G, Verhey F. Awareness in dementia: A review of clinical correlates. Aging \& Mental Health 2005;9:414-422.

5. Okonkwo OC, Spitznagel MB, Alosco ML, Tremont G. Associations among measures of awareness of cognitive deficits in dementia. Alzheimer's \& ${ }^{2}$ Dementia 2010;6:312-318.

6. Vasterling JJ, Seltzer B, Foss JW, Vanderbrook V. Unawareness of deficit in Alzheimer's disease: Domain-specific differences and disease correlates. Neuropsychiatry, Neuropsychology, \&3 Behavioral Neurology 1995;8:26-32.

7. Clare L. Developing awareness about awareness in early-stage dementia: The role of psychosocial factors. Dementia: The International Journal of Social Research and Practice 2002;1:295-312.

8. Aalten P, van Valen E, de Vugt ME, Lousberg R, Jolles J, Verhey FRJ. Awareness and behavioral problems in dementia patients: A prospective study. International Psychogeriatrics 2006;18:3-17.

9. Reed J, Cantley C, Clarke CL, Stanley D. Services for younger people with dementia: Problems with differentiating needs on the basis of age. Dementia: The International Journal of Social Research and Practice 2002;1:95-112.

10. Beattie AM, Daker-White G, Gilliard J, Means R. Younger people in dementia care: a review of service needs, service provision and models of good practice. Aging $\mathcal{E}$ Mental Health 2002;6:205-212.

11. Verhey FR, Rozendaal N, Ponds RW, Jolles J. Dementia, awareness and depression. 1993;8:851-856.

12. Lopez OL, Becker JT, Somsak D, Dew MA. Awareness of cognitive deficits and anosognosia in probable Alzheimer's disease. European Neurology 1994;34:277-282.

13. Harris PB, Keady J. Living with early onset dementia: exploring the experience and developing evidence-based guidelines for practice. Alzheimer's Care Quarterly $2004 ; 5: 111-122$.

14. van Vliet D, Bakker C, Koopmans RT, Vernooij-Dassen MJ, Verhey FR, de Vugt ME. Research protocol of the NeedYD-study (Needs in Young onset Dementia): a prospective cohort study on the needs and course of early onset dementia. BMC Geriatrics 2010;10:13.

15. American Psychiatric Association. Diagnostic and Statistical manual of mental Disorders- Text revision: DSM-IV-TR. American Psychiatric Association 2000. 
16. CBO: Guideline diagnosis and pharmacological treatment of dementia. 2005. Available at: http://www.cbo.nl/Downloads/387/rldement2005.pdf. Accessed: March 3, 2012.

17. McKhann G, Drachman D, Folstein M, Katzman R, Price D, Stadlan EM. Clinical diagnosis of Alzheimer's disease: report of the NINCDS-ADRDA Work Group under the auspices of Department of Health and Human Services Task Force on Alzheimer's Disease. Neurology 1984;34:939-944.

18. McKeith IG. Consensus guidelines for the clinical and pathologic diagnosis of dementia with Lewy bodies (DLB): report of the Consortium on DLB International Workshop. Journal of Alzheimer's Disease 2006;9:417-423.

19. Neary D, Snowden JS, Gustafson L, et al. Frontotemporal lobar degeneration: a consensus on clinical diagnostic criteria. Neurology 1998;51:1546-1554.

20. Mesulam MM, Grossman M, Hillis A, Kertesz A, Weintraub S. The core and halo of primary progressive aphasia and semantic dementia. Annals of Neurology 2003;54 Suppl 5:S11-14.

21. Erkinjuntti T. Clinical criteria for vascular dementia: The NINDS-AIREN criteria. Dementia 1994;5:189-192.

22. de Vugt ME, Jolles J, van Osch L, et al. Cognitive functioning in spousal caregivers of dementia patients: findings from the prospective MAASBED study. Age and Ageing 2006;35:160-166.

23. Zanetti O, Vallotti B, Frisoni GB, et al. Insight in dementia: When does it occur? Evidence for a nonlinear relationship between insight and cognitive status. Journals of Gerontology: Series B: Psychological Sciences and Social Sciences 1999:100-106.

24. Cummings JL, Mega M, Gray K, Rosenberg-Thompson S. The Neuropsychiatric Inventory: Comprehensive assessment of psychopathology in dementia. Neurology 1994;44:2308-2314.

25. Kat MG, de Jonghe JFM, Aalten P, Kalisvaart CJ, Dres RM, Verhey FRJ. Neuropsychiatrische symptomen bij dementie: Psychometrische aspecten van de Nerlandse Neuropsychiatric Inventory (NPI). Tijdschrift voor Gerontologie en Geriatrie 2002;33:150-155.

26. Feher EP, Mahurin RK, Inbody SB, Crook TH. Anosognosia in Alzheimer's disease. Neuropsychiatry, Neuropsychology, \&s Behavioral Neurology 1991;4:136-146.

27. Reed BR, Jagust WJ, Coulter L. Anosognosia in Alzheimer's disease: Relationships to depression, cognitive function, and cerebral perfusion. Journal of Clinical and Experimental Neuropsychology 1993;15:231-244.

28. Starkstein SE, Chemerinski E, Sabe L, Kuzis G. Prospective longitudinal study of depression and anosognosia in Alzheimer's disease. British Journal of Psychiatry 1997;171:47-52.

29. Arkin S, Mahendra N. Insight in Alzheimer's patients: Results of a longitudinal study using three assessment methods. American Journal of Alzheimer's Disease 2001;16:211-224. 
30. van der Flier WM, Pijnenburg YAL, Fox NC, Scheltens P. Early-onset versus lateonset Alzheimer's disease: The case of the missing APOE E4 allele. The Lancet Neurology 2010;10:280-288.

31. Stewart G, McGeown WJ, Shanks MF, Venneri A. Anosognosia for memory impairment in Alzheimer's disease. Acta Neuropsychiatrica 2010;22:180-187.

32. Drzezga A, Grimmer T, Henriksen G, et al. Effect of APOE genotype on amyloid plaque load and gray matter volume in Alzheimer disease. Neurology 2009;72:14871494.

33. Rosen HJ. Anosognosia in neurodegenerative disease. Neurocase 2011;17:231-241.

34. Starkstein SE, Sabe L, Chemerinski E, Jason L, Leiguarda R. Two domains of anosognosia in Alzheimer's disease. Journal of Neurology, Neurosurgery, and Psychiatry 1996;61:485-490.

35. Migliorelli R, Tesn A, Sabe L, Petracchi M. Prevalence and correlates of dysthymia and major depression among patients with Alzheimer's disease. The American Journal of Psychiatry 1995;152:37-44.

36. Seignourel PJ, Kunik ME, Snow L, Wilson N, Stanley M. Anxiety in dementia: a critical review. Clinical Psychology Review 2008;28:1071-1082.

37. Derouesne C, Thibault S, Lagha-Pierucci S, Baudouin-Madec V, Ancri D, Lacomblez L. Decreased awareness of cognitive deficits in patients with mild dementia of the Alzheimer type. International Journal of Geriatric Psychiatry 1999;14:10191030 .

38. Dux MC, Woodard JL, Calamari JE, et al. The moderating role of negative affect on objective verbal memory performance and subjective memory complaints in healthy older adults. Journal of the International Neuropsychological Society 2008;14:327-336.

39. Mangone CA, Hier DB, Gorelick PB, Ganellen RJ. Impaired insight in Alzheimer's disease. 1991;4:189-193.

40. Williamson C, Alcantar O, Rothlind J, Cahn-Weiner D, Miller BL, Rosen HJ. Standardised measurement of self-awareness deficits in FTD and AD. Journal of Neurology, Neurosurgery \& Psychiatry 2010;81:140-145.

41. Clare L, Wilson BA, Carter G, Roth I, Hodges JR. Awareness in Early-Stage Alzheimer's Disease: Relationship to Outcome of Cognitive Rehabilitation. Journal of Clinical and Experimental Neuropsychology 2004;26:215-226. 

Chapter 8

\section{General discussion}


The central goal of this thesis was to further our understanding of the impact and clinical characteristics of YOD and to assess whether these differ from LOD. Three aspects in particular were investigated: (1) the psychosocial impact of dementia on caregivers, (2) diagnostic issues and (3) neuropsychiatric symptoms. In this chapter, the main findings will be summarized by addressing these three areas. The methodological and conceptual issues of the NeedYD study are discussed as well as the implications for clinical practice. Recommendations are made for future studies and the chapter ends with a general conclusion.

\subsection{Summary of findings}

\section{The psychosocial impact of YOD on caregivers}

In chapter 3, we assessed the psychosocial impact of YOD on caregivers and investigated whether this impact differs from that in LOD by means of a systematic literature review. The results indicated that YOD caregivers experience high levels of burden and stress, and the majority of caregivers suffered from depression. Only two studies compared the psychological outcome of YOD and LOD caregivers. No firm conclusions could be drawn based on these studies because of contradicting results and methodological limitations. However, important life domains in YOD caregivers were shown to be negatively affected. Common themes were a changing marital relationship, family conflict, problems with workforce participation and finances, diagnostic difficulties and a lack of designated services. In terms of the caregiver stress model as proposed by Pearlin et al. (1990) and discussed in the introduction of this thesis, it can be concluded that differences in caregiver outcome are unclear. The secondary role strains such as job-caregiving conflict, economic problems and conflicts with children living in the same household are more specific for YOD caregivers, at least when spousal caregivers are considered. Diagnostic difficulties and a lack of designated services may be viewed as contextual factors more specific for $\mathrm{YOD}^{1-3}$, but more research on these topics is necessary. None of the reported studies specifically focused on the impact of YOD on children. In summary, the review showed that the psychosocial impact of YOD is high, but whether the impact on caregivers differs between YOD and LOD is still unclear. Nevertheless, YOD caregivers do experience high levels of psychological suffering and specific problems related to their phase in life; therefore, there is an urgent need to expand research on this topic.

\section{Barriers to YOD diagnosis}

In light of the previous and the increasing importance of an early diagnosis, we investigated the time from symptom onset to diagnosis in YOD and LOD patients and attempted to determine the factors that influence this duration. We showed in chapter 4 that it took on average 1.6 years longer from symptom onset to diagnosis in YOD patients than in LOD. A younger age at onset and an FTD diagnosis 
were predictive of an extended time between symptom onset and diagnosis, with younger age at onset being the strongest predictor. To obtain more information regarding the barriers to diagnosis, we analyzed qualitative interviews with 92 YOD caregivers (chapter 5). Five themes emerged relating to a delay in diagnosis: (1) "Misattribution of symptoms by caregivers", (2) "Denial of the individual with YOD and the refusal to seek medical advice", (3) "Lack of confirmation from the social context", (4) "Lack of responsiveness of the general practitioner" and (5) "Misdiagnosis leading to inadequate advice/help". It is unclear in what way these issues differ from the situation in LOD and to what extent each theme actually leads to a diagnostic delay. However, the factors and dynamics leading to a delay are hypothesized to be different between YOD and LOD. In addition, the impact of a delay in diagnosis appeared to be higher in YOD patients than in LOD. In YOD, a lack of knowledge and understanding regarding the cause of changing behavior resulted in early discharge from work, financial hardship, disrupted relationships between spouses or between parents and children, and in some cases, almost to divorce. Diagnosis therefore marks an important turning point because it provides caregivers with an explanatory model and places events in the proper perspective.

\section{Neuropsychiatric symptoms and level of awareness}

Behavioral problems are more common in YOD because of the different etiologies occurring at a younger age ${ }^{4,5}$, but it is yet unknown whether YOD and LOD show different neuropsychiatric profiles within diagnostic categories. Therefore, the frequency parameters of neuropsychiatric symptoms were compared between YO-AD and LO-AD in chapter 6 . We found that over the course of two years, the incidence, prevalence and persistence of neuropsychiatric symptoms was overall lower in YO-AD, with significant differences for delusions, agitation, depression, anxiety, apathy, irritability and aberrant motor behavior. The frequency of individual symptoms showed large variability. The most frequent symptom in YO-AD was apathy. The profiles of neuropsychiatric symptoms were roughly similar between groups, but eating changes ranked higher and delusions ranked lower in YO-AD. Contrary to our expectations, affective symptoms were less frequent in YO-AD compared with LO-AD. Because affective symptoms may be mediated by level of awareness $^{6,7}$, we investigated differences in awareness between both groups and the association with affective symptoms in chapter 7 . We demonstrated that YOAD patients had higher levels of awareness than LO-AD patients over a one-year period. High levels of awareness were associated with depression but not anxiety over the study period. No overall interaction-effect was found, but at baseline, YO-AD patients with high awareness experienced more depressive symptoms compared to LO-AD patients and patients with low awareness. A causal relationship between awareness and affective symptoms could not be established. 


\subsection{Young-onset dementia patients as a separate group}

The rationale behind the NeedYD study was the increasing awareness that YOD patients may represent a specific group, whose needs may differ from LOD patients. We expected differences between these groups based on differences in agerelated contextual factors. Hence, the question arises as to why age is specifically important in dementia care and if YOD is a valid concept. First, a biological argument needs to be considered. In YOD, rare, hereditary, metabolic and treatable causes, FTD and secondary dementias are more common. Furthermore, differences in clinical symptoms, prognosis, genetic factors, neuropathology, and neurochemical deficits between YO-AD and LO-AD have been shown ${ }^{8}$. Currently, evidence is accumulating that cerebrovascular disease plays a causal role in LO$\mathrm{AD}$, which differs from the pure neurodegenerative disease observed in younger $\mathrm{AD}$ patients ${ }^{9,10}$, suggesting the existence of two categories. In addition, within nonfamilial YO-AD, patients with a distinct $\mathrm{AD}$ subtype have been identified with a non-memory phenotype, more widespread atrophy beyond the temporal lobe, greater hypometabolism in the posterior regions and negativity for the APOE $\varepsilon 4$ allele ${ }^{11-13}$. The heterogeneity in YOD etiologies and within diagnostic categories such as YO-AD thus necessitates careful diagnostic investigation and the provision of specific treatment options.

Second, there is a practical basis for a distinction between YOD and LOD. Dementia care services are almost universally age specific, catering for older people with dementia ${ }^{8}$, and, in some countries, these services exclude patients under the age of 65. In addition, dementia is typically viewed as an 'old person's disease', and YOD patients present a minority among the large group of elderly suffering from dementia. Therefore, specialized services will prevent YOD patients from feeling marginalized with regard to their social life and services ${ }^{14,15}$. However, one may argue that services should generally be more responsive to individual needs instead of catering specifically to YOD patients ${ }^{16,17}$. Admittedly, a strictly dichotomous view in clinical practice is somewhat arbitrary, as the experience of someone who is 64 years of age is not necessarily different from that of a person aged 66. However, the evidence arguing for a specific approach in the care for YOD patients and their families is growing. Our findings add to this knowledge base and show differences between YOD and LOD with important clinical implications. Although the young-onset dementias may not have validity as diagnostic categories because age cannot be seen as a defining characteristic between two qualitatively different disease entities in dementia, we argue that the concept of YOD does have utility because it helps clinicians make decisions regarding patient management and treatment ${ }^{18}$. 


\subsection{Methodological considerations}

\section{Sample selection}

Sample bias could have been a factor in our study because patients were recruited through a large range of different institutions instead of the community. Furthermore, the group of patients that consented to the study may have been different from the group of patients that refused to participate. YOD patients and caregivers experiencing higher levels of burden, more severe behavioral disturbances or cognitive impairment may be more likely to refuse. The generalizability of the results to the whole YOD population is therefore limited. In addition, several diagnostic categories were excluded from our study, i.e., dementia due to HIV, traumatic brain injury, Down syndrome, Huntington's disease or alcohol-related dementia, further limiting the generalizability of the results. However, these diagnoses are associated with a range of other specific problems, which would have obscured the problems related to a younger age at onset, and these diagnoses were therefore excluded.

Although we took care in including patients who met the clinical criteria for dementia and had an age of onset before 65, not all etiological diagnoses could be confirmed with medical records. However, in the Netherlands, specific consensus guidelines for establishing a dementia diagnosis ${ }^{19}$ and guidelines for referral ${ }^{20}$ in primary care are commonly used. In these guidelines, referral in the case of suspicion of YOD and the use of neuroimaging and biomarker research is recommended specifically in patients under the age of 65 . Therefore, we expect this to be of minor influence on our results.

Furthermore, defining a sample of YOD and LOD is by nature subject to bias, as the criterion to divide these patients is based on the recollections of patients and caregivers. This is an inevitable drawback of this type of study. We argue that this drawback is not a major influence on our conclusions because we aimed at distinguishing the general experience of YOD patients and their caregivers versus those with LOD.

\section{Study design}

The longitudinal study design of the NeedYD study has the advantage of studying changes over time and allowing causal inferences to be determined. Adding a temporal component to observational studies can provide support for a theorized cause and effect because a causal factor precedes an effect in time. A disadvantage of longitudinal study designs is selective attrition due to early death and dropout. Restricting analyses to participants with complete follow-up potentially leads to biased results because patients in the more severe stages of dementia or caregivers with high burden levels are the most likely to drop out. Therefore, we used multiple imputation of missing values when feasible, which is currently considered the state-of-the-art method ${ }^{21}$. In chapter 6 , multiple imputations of missing data 
were not possible due to the descriptive nature of this study. Therefore, the characteristics of study completers were compared to study dropouts. The patients who dropped out of the study were older and more cognitively impaired. The results were therefore restricted to a selection of patients with mildly disturbed cognitive function and younger age. Consequently, the presence of behavioral disturbances could have been underestimated.

In addition, a naturalistic study design essentially does not allow for interference with the natural setting such as treatment and medication use. Psycho-active medication use appeared to differ between the YOD and LOD group. Service use was not assessed at baseline in MAASBED, but was probably lower because the NeedYD study recruited patients through day-care centers. Furthermore, the use of other psychosocial services by patients and caregivers varied considerably across participants ${ }^{22}$. A possible confounding effect of these factors on the course of behavioral disturbances and the difference between $\mathrm{YO}-\mathrm{AD}$ and $\mathrm{LO}-\mathrm{AD}$ therefore cannot be precluded. The more frequent use of anti-dementia medication, which appears to have a modest positive effect on behavioral disturbances ${ }^{23-25}$, or the more frequent use of psychosocial services in the YOD group ${ }^{26}$ might therefore partly explain the lower NPI scores in this group.

\section{Group comparisons}

The data from the NeedYD study and the cohort of the LOD sample (MAASBED study) were different in several ways, as the participants were not matched. While both studies sampled patients from memory clinics and institutes for community mental health using essentially the same design, assessment measures and diagnostic criteria, the MAASBED study only included consecutive referrals after diagnosis, while the NeedYD study also included patients using specialized day care. The YOD group was, therefore, more heterogeneous in terms of disease severity, disease duration and cognitive functioning. In our analyses, we corrected for the differences in demographics and dementia severity. We included the GDS in our analyses as a measure of global severity of dementia because this accounts for a broad range of relevant domains such as disabilities in daily living, behavioral and cognitive functioning. Moreover, the MMSE had missing values in a large sample of the YOD patients and could therefore not be used in the analyses.

Strictly speaking, a mere comparison of YOD and LOD groups cannot answer the question of whether the consequences of dementia for caregivers and patients are different between groups without the use of healthy control groups. The relative increased risk of affective symptoms in dementia patients compared to controls may be higher in the YOD group than in the LOD group. Affective symptoms may already be higher in $\mathrm{LOD}^{27,28}$, explaining the difference we have found. However, for clinical practice, it is important to explore these potential differences between patient groups because it may provide guidance in clinical practice. 


\section{Assessment methods}

As mentioned previously, the moment of symptom onset in dementia is difficult to determine because of its inevitable retrospective estimation and the insidious onset of dementia. Our studies on the period prior to diagnosis are strengthened by the use of both quantitative and qualitative research methods. While quantitative research methods are generally viewed as more reliable and generalizable, qualitative research methods provide rich insights into the experiences of people and illuminates context.

In the NeedYD study, the Neuropsychiatric Inventory (NPI) was used as a measure of neuropsychiatric symptoms, which is the most widely used rating scale to assess neuropsychiatric symptoms in dementia and has established validity and reliability ${ }^{29}$. Although a wide range of behavioral disturbances is measured by the NPI, it does not cover all behavioral symptoms occurring in dementia. The validity of our results is therefore limited to the symptoms included in the NPI. Furthermore, it is a general instrument, and a more extensive assessment for specific symptoms would have been more appropriate such as the Cohen-Mansfield agitation inventory $^{30}$, the apathy evaluation scale ${ }^{31}$ and the Cornell scale for depression ${ }^{32}$. However, because of the exploratory nature of the studies presented in this thesis and the aim of assessing a broad range of symptoms, the NPI was most appropriate and feasible.

The NPI is generally used to assess specific neuropsychiatric symptoms, which can be grouped into behavioral sub-syndromes. However, the results of factor analyses of the NPI are not consistent across studies and have not been conducted in YOD patients ${ }^{33}, 34$. Furthermore, the sub-syndromes are by definition dependent on the items included in the assessment instrument. The inclusion of, for example, shouting, personality changes and sexual disturbances in the NPI assessment might lead to other sub-syndromes ${ }^{33}$. Because little is known regarding the presence of the neuropsychiatric symptoms and syndromes in YOD, we preferred to use the individual NPI item scores instead of possible sub-syndromes.

One may argue that proxy ratings are not an objective measurement of behavioral problems. Caregiver reports may be influenced by feelings of burden or depression, caregiver denial or educational leve ${ }^{35}$. In addition, the NPI is susceptible to recall bias, as it focuses on the behavioral disturbances occurring within the preceding month. We chose this method because dementia patients gradually become cognitively impaired, may suffer from a lack of awareness and may therefore be unable to report their symptoms. New research methods such as experience sampling methods ${ }^{36}$ or dementia care mapping ${ }^{37}$ might provide a more accurate picture of the occurrence of neuropsychiatric symptoms.

The guidelines for the rating of awareness deficits (GRAD) were used to assess levels of awareness. This is a global rating scale, which has several practical advantages. However, domain-related variations in awareness are not likely to be noted. In addition, the scale relies heavily on explicit verbal responses and therefore does not take into account implicit awareness as displayed through behavior. 
Furthermore, some degree of interpretation of the researcher is necessary, which is likely to influence the results ${ }^{38}$. However, these issues will occur in both the YOD and LOD groups, allowing for a fair comparison between groups. Furthermore, the assessment methods in NeedYD were chosen in line with the study protocol of the MAASBED-study. This gave us the unique opportunity to compare these outcomes in a large group of YOD and LOD patients.

\subsection{Clinical implications}

Our findings underline the specific importance of an earlier diagnosis in YOD. Knowing the nature and background of the difficulties experienced may help YOD caregivers and patients better understand and cope with their burdening situation; this knowledge may prevent discharge from work, divorce or disrupted relationships with children. Because many caregivers felt poorly understood by the medical profession, we believe that efforts should be made to promote awareness of YOD in primary care. Our recommendations are mainly aimed at improving clinician awareness to the possibility of dementia at a young age, so YOD is consequently included in the possible differential diagnoses.

First, awareness of YOD should be promoted among primary care physicians and occupational physicians, as they are the first to be confronted with these patients. Second, community mental health services are an important target because we have shown that symptoms are frequently labeled as psychological or psychiatric, and patients are referred to mental health services for counseling or treatment. Especially in the cases of treatment non-response, alertness to possible YOD is necessary. Furthermore, it is important that clinicians respond to the individual needs of caregivers and ensure the regular follow-up of people presenting with cognitive and/or behavioral changes. In addition, the emphasis should lie on the reports of proxies next to reports of patients, especially when dementia is suspected.

Furthermore, our results showed that behavioral disturbances are overall less common in YO-AD than in LO-AD but are still common in this group, especially apathy. These symptoms are therefore an important part of the diagnostic process, treatment, support and psycho-education of YO-AD patients and caregivers. The results also indicated an overall higher level of awareness in YO-AD patients and a higher prevalence of depressive symptoms in patients with high levels of awareness. Caregivers and clinicians should thus be prepared for affective symptoms in YO$\mathrm{AD}$ patients with high awareness. YO-AD patients will also more often have the capability and willingness to be actively involved in decisions surrounding the care process and should therefore be given that opportunity. Preserved insight further enables patients to take advantage of information provision, peer support groups and psychological treatments when the need exists. In addition, treatment compliance appears higher and cognitive rehabilitation more effective in patients with awareness ${ }^{39}$. 


\section{Implications for services}

Our review showed that the impact and problems experienced by YOD caregivers are of a different nature than those of LOD caregivers. Because of the presence of younger children, patient and caregiver workforce participation and the specific financial issues in YOD families, a family-centered care approach is more important than in LOD. Furthermore, the clinical characteristics are different in YOD (i.e., behavioral disturbances and awareness). Because of the low prevalence of YOD, most health care workers are by definition not well experienced with the specific problems of this patient group. In our view, specialized services for YOD patients and their caregivers with well-educated and trained health care workers will therefore be better suited to support YOD patients and their families than the traditional services.

We argue for the development of specific support and respite services for YOD patients and their caregivers, the inclusion of YOD as a topic in existing educational programs and the development of supplementary training programs for health care workers involved in the care of YOD patients. Respite care is of importance in YOD because of the dual tasks YOD caregivers have of providing care for their spouse while maintaining a job and caring for their children. Our finding that awareness levels are higher in YOD also implies that psychological support, information provision and patient empowerment should be key issues in the service provision for YOD patients. The NeedYD study will soon provide important information on the specific needs of YOD patients and caregivers; this information is necessary to develop more specific guidelines for care services.

In addition, policy should focus on developing and/or enhancing specialized diagnostic centers for the diagnosis of YOD. This in turn will raise awareness of this subgroup among the medical profession and streamline the diagnostic process. Referral to a specialized memory clinic is important ${ }^{40}$ because a structured approach based on all clinical features is required to diagnose YOD ${ }^{41}$. Furthermore, such services will allow expertise to increase and can serve as a gateway to coordinated service provision for YOD patients and their caregivers ${ }^{42}$.

\subsection{Further research}

Our review showed that high-quality research on the differences in impact between YOD and LOD caregivers is scarce. Recommendations for future research include cohort studies that compare functioning, experiences, needs and risk factors for negative caregiver outcome of YOD caregivers with LOD caregivers during the course of the illness. The themes that emerged from the review (i.e., the financial hardship, relational problems, diagnostic process, family conflict, and children's negative experiences) should be investigated in more detail to adequately support caregivers. Because research on LOD showed behavioral disturbances to be a major contributor to patients and caregiver suffering, the impact of these symptoms 


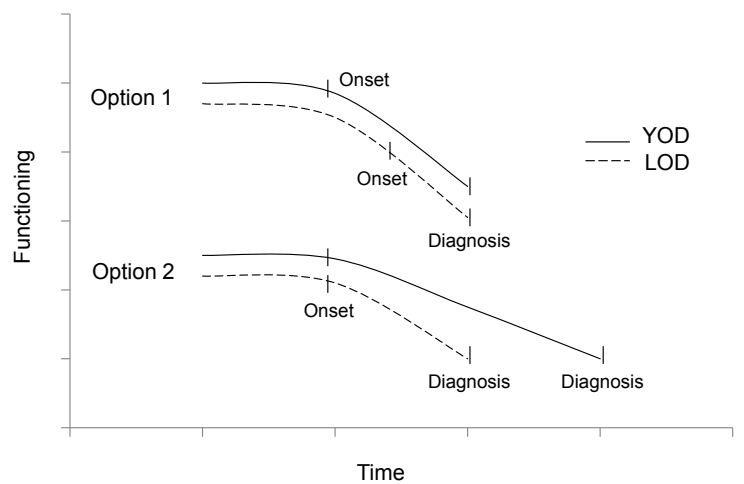

Figure 8.1: Possible course of functioning in YOD and LOD before diagnosis, with a delayed onset as noticed by caregivers in LOD in option 1, and a less progressive decline of functioning in YOD in option 2, explaining the results of a longer time to diagnosis in YOD and a comparable disease severity at the time of diagnosis.

in YOD should be the focus of future research. In this respect, the NeedYD study will provide important insights in the near future.

In addition, with the advances in biomarker research, the etiology of dementia can be established with increasing accuracy. A great deal of research has focused on improving etiologic diagnostics in YOD, as diagnosing YOD is particularly problematic due to the heterogeneity of dementia etiologies at a younger age. However, the psychosocial factors related to delays in help-seeking behavior and diagnosis for YOD should not be overlooked. This thesis illustrated the key issues for future research, i.e., the denial and the refusal to seek help of the YOD patient, the misattribution of symptoms, inadequate professional help and faulty diagnoses. Furthermore, our findings indicated that the duration from symptom onset to diagnosis was longer in YOD than in LOD, but dementia severity was comparable at the time of diagnosis. The possible explanations for our results are depicted in figure 1 and deserve further investigation in more homogeneous YOD and LOD samples. Noting the onset of dementia may be masked by expected age-related cognitive decline in LOD, or subtle initial symptoms may be noted earlier in YOD because of the higher environmental demands (option 1). Another possibility is based on the theory of cognitive reserve, which hypothesizes that anatomical features and neuronal networks can actively compensate after damage to the brain ${ }^{43,44}$. YOD patients may be better able to compensate for their cognitive impairment and therefore show a less progressive decline in functioning during the very early stages of the disease (option 2).

Future studies should also aim at further characterizing the clinical profile of YOD patients, which should expand to institutionalized patients and other diagnostic subtypes than AD. Within YO-AD, currently, a great deal of work focuses 
on the identification of different AD-subtypes, but still little is known about why some patients have an atypical clinical presentation and why this occurs at a younger age of onset ${ }^{45}$. Furthermore, no agreement exists on the terminology and classification of these subtypes ${ }^{45}$. Like other researchers, we have hypothesized that a lower rate of behavioral disturbances and impaired awareness in YO-AD could be related to the presence of these specific AD subtypes based on studies showing typical amyloid deposition patterns and APOE genotypes ${ }^{11}$. However, these findings have been contradicted by others ${ }^{46}$. Hence, investigating the relationship between pathological and genetic features and clinical outcome in YO-AD and defining consensus criteria for each subtype will prove important for diagnostic accuracy, clinical trials, the development of targeted interventions, and clinical management ${ }^{47}$.

The next step would be to perform intervention studies to test the efficacy of specialized services and care approaches for YOD patients and their caregivers. Younger people with dementia possibly have resources beyond those of older persons with dementia. Psychosocial interventions or cognitive training may be more beneficial in younger patients because of the lower rate of behavioral symptoms, their relatively preserved awareness, memory capacity and better general health condition. Furthermore, younger people may have a larger social network and may be psychologically more resilient than the elderly. Research should therefore focus on the possibilities and resources of YOD patients and caregivers and how to optimally employ them and the potential benefits of interventions.

\subsection{General conclusions}

The present thesis shows that there are important differences in impact and clinical characteristics between YOD and LOD. YOD has a large impact on caregivers and negatively affects multiple life domains related to their earlier phase in life. The pre-diagnostic period is longer and appears more problematic in YOD. Furthermore, a delay in diagnosis has a higher detrimental impact on daily life in YOD, indicating that efforts should be made to provide an earlier diagnosis. In addition, YO-AD patients show higher levels of awareness, implying that they are better able to take advantage of psychosocial interventions and can be actively involved in decisions regarding their care process. Behavioral symptoms are less frequent in YO-AD, but these symptoms, notably apathy, should still receive sufficient attention in YO-AD patients. Further research is necessary to investigate the biopsychosocial dynamics underlying the differences in clinical characteristics and to assess the effects of psychosocial interventions in YOD. 


\section{References}

1. Harris PB. The Perspective of Younger People with Dementia: Still an Overlooked Population. Social Work in Mental Health 2004;2:17-36.

2. Barber R. A survey of services for younger people with dementia. International Journal of Geriatric Psychiatry 1997;12:951-954.

3. Werner P, Stein-Shvachman I, Korczyn AD. Early onset dementia: clinical and social aspects. International Psychogeriatrics 2009:1-6.

4. Mendez MF. The accurate diagnosis of early-onset dementia. International Journal of Psychiatry in Medicine 2006;36:401-412.

5. Diehl J, Forstl H, Jansen S, Kurz A. Frontotemporal dementia: specific problems for caregivers. Zeitschrift fur Gerontologie und Geriatrie 2004;37:301-306.

6. Aalten P, van Valen E, de Vugt ME, Lousberg R, Jolles J, Verhey FRJ. Awareness and behavioral problems in dementia patients: A prospective study. International Psychogeriatrics 2006;18:3-17.

7. Aalten P, Van Valen E, Clare L, Kenny G, Verhey F. Awareness in dementia: A review of clinical correlates. Aging \& Mental Health 2005;9:414-422.

8. Harvey RJ, Rossor MN, Skelton-Robinson M, Garralda E. Young onset dementia: epidemiology, clinical symptoms, family burden, support and outcome. Imperial college of science, technology and medicine Dementia research group 1998.

9. Richards M, Brayne C. What do we mean by Alzheimer's disease? British Medical Journal 2010;341:c4670.

10. Richard E, Van Gool WA. Alzheimer's disease/syndrome? There are two types of disease. British Medical Journal 2010;341:6327.

11. van der Flier WM, Pijnenburg YAL, Fox NC, Scheltens P. Early-onset versus lateonset Alzheimer's disease: The case of the missing APOE E4 allele. The Lancet Neurology 2010;10:280-288.

12. Pijnenburg YA, Zeeman-Rebel A, van der Flier WM, et al. Cognitive disorders appearing before the age of 65 in patients of the Alzheimer Centre of the VU Medical Centre: diagnoses and clinical characteristics. Nederlands Tijdschrift voor Geneeskunde 2005;149:2862-2867.

13. Lehmann M, Koedam EL, Barnes J, et al. Posterior cerebral atrophy in the absence of medial temporal lobe atrophy in pathologically-confirmed Alzheimer's disease. Neurobiology of Aging 2012;33:6271-62712.

14. Beattie A, Daker-White G, Gilliard J, Means R. 'They don't quite fit the way we organize our services'--Results from a UK field study of marginalized groups and dementia care. Disability \& Society 2005;20:67-80.

15. Harris PB, Keady J. Living with early onset dementia: exploring the experience and developing evidence-based guidelines for practice. Alzheimer's Care Quarterly 2004;5:111-122.

16. Beattie AM, Daker-White G, Gilliard J, Means R. Younger people in dementia care: a review of service needs, service provision and models of good practice. Aging \& Mental Health 2002;6:205-212. 
17. Reed J, Cantley C, Clarke CL, Stanley D. Services for younger people with dementia: Problems with differentiating needs on the basis of age. Dementia: The International Journal of Social Research and Practice 2002;1:95-112.

18. Kendell R, Jablensky A. Distinguishing between the validity and utility of psychiatric diagnoses. The American Journal of Psychiatry 2003;160:4-12.

19. CBO: Guideline diagnosis and pharmacological treatment of dementia. 2005. Available at: http://www.cbo.nl/Downloads/387/rldement2005.pdf. Accessed: March 3, 2012.

20. NHG Clinical Practice Guidelines M21 Dementia. 2003. Dutch College of General Practitioners. Available at: http://nhg.artsennet.nl.

21. Schafer JL, Graham JW. Missing data: our view of the state of the art. Psychological Methods 2002;7:147-177.

22. Bakker C, de Vugt ME, van Vliet D, et al. The use of formal and informal care in early onset dementia: results from the NeedYD study. American Journal of Geriatric Psychiatry 2012 [Epub ahead of print].

23. Sink KM, Holden KF, Yaffe K. Pharmacological Treatment of Neuropsychiatric Symptoms of Dementia: A Review of the Evidence. Journal of the American Medical Association 2005;293:596-608.

24. Kavanagh S, Gaudig M, Van Baelen B, et al. Galantamine and behavior in Alzheimer disease: Analysis of four trials. Acta Neurologica Scandinavica 2011;124:302308.

25. Cummings JL, Mackell J, Kaufer D. Behavioral effects of current Alzheimer's disease treatments: A descriptive review. Alzheimer's \& Dementia 2008;4:49-60.

26. Droes RM, Meiland F, Schmitz M, van Tilburg W. Effect of combined support for people with dementia and carers versus regular day care on behaviour and mood of persons with dementia: results from a multi-centre implementation study. International Journal of Geriatric Psychiatry 2004;19:673-684.

27. Hoyert DL, Heron MP, Murphy SL, Kung HC. Deaths: final data for 2003. National Vital Statistics Reports 2006;54:1-120.

28. Stordal E, Mykletun A, Dahl AA. The association between age and depression in the general population: A multivariate examination. Acta Psychiatrica Scandinavica 2003;107:132-141.

29. Cummings JL, Mega M, Gray K, Rosenberg-Thompson S. The Neuropsychiatric Inventory: Comprehensive assessment of psychopathology in dementia. Neurology 1994;44:2308-2314.

30. de Jonghe JFM, Kat MG. Factor structure and validity of the Dutch version of the Cohen-Mansfield Agitation Inventory (CMAI-D). Journal of the American Geriatrics Society 1996;44:888-889.

31. Marin RS, Biedrzycki RC, Firinciogullari S. Reliability and validity of the Apathy Evaluation Scale. Psychiatry Research 1991;38:143-162.

32. Alexopoulos GS, Abrams RC, Young RC, Shamoian CA. Cornell Scale for Depression in Dementia. Biological Psychiatry 1988;23:271-284. 
33. Aalten P, Verhey FR, Boziki M, et al. Neuropsychiatric syndromes in dementia. Results from the European Alzheimer Disease Consortium: part I. Dementia and Geriatric Cognitive Disorders 2007;24:457-463.

34. Garre-Olmo J, Batlle DG, del Mar Fernandez M, et al. Incidence and subtypes of early-onset dementia in a geographically defined general population. Neurology 2010;75:1249-1255.

35. de Medeiros K, Robert P, Gauthier S, et al. The Neuropsychiatric InventoryClinician rating scale (NPI-C): Reliability and validity of a revised assessment of neuropsychiatric symptoms in dementia. International Psychogeriatrics 2010;22: 984-994.

36. Brown LH, Strauman T, Barrantes-Vidal N, Silvia PJ, Kwapil TR. An experiencesampling study of depressive symptoms and their social context. Journal of Nervous and Mental Disease 2011;199:403-409.

37. Sloane PD, Brooker D, Cohen L, et al. Dementia care mapping as a research tool. International Journal of Geriatric Psychiatry 2007;22:580-589.

38. Clare L, Markova I, Verhey F, Kenny G. Awareness in dementia: A review of assessment methods and measures. Aging $\&$ Mental Health 2005;9:394-413.

39. Clare L, Wilson BA, Carter G, Roth I, Hodges JR. Awareness in Early-Stage Alzheimer's Disease: Relationship to Outcome of Cognitive Rehabilitation. Journal of Clinical and Experimental Neuropsychology 2004;26:215-226.

40. Sampson EL, Warren JD, Rossor MN. Young onset dementia. Postgraduate Medical Journal 2004;80:125-139.

41. Rossor MN, Fox NC, Mummery CJ, Schott JM, Warren JD. The diagnosis of young-onset dementia. Lancet Neurology 2010;9:793-806.

42. Williams T, Cameron I, Deardon T. From pillar to post-a study of younger people with dementia. Psychiatric Bulletin 2001;25:384-387.

43. Stern Y. Cognitive reserve. Neuropsychologia 2009;47:2015-2028.

44. Fairjones SE, Vuletich EJ, Pestell C, Panegyres PK. Exploring the role of cognitive reserve in early-onset dementia. American Journal of Alzheimer's Disease and Other Dementias 2011;26:139-144.

45. Llado A, Sanchez-Valle R. Focusing on atypical symptoms for improved diagnosis of early-onset Alzheimer's disease. Future Neurology 2011;6:575-578.

46. Crutch SJ, Lehmann M, Schott JM, Rabinovici GD, Rossor MN, Fox NC. Posterior cortical atrophy. Lancet Neurology 2012;11:170-178.

47. Schott JM, Ridha BH, Crutch SJ, et al. Apolipoprotein e genotype modifies the phenotype of Alzheimer disease. Archives of Neurology 2006;63:155-156. 


\section{Summary}

There is increasing awareness that YOD patients represent a specific group in health care, whose needs may differ from those of LOD patients. However, research on this top is scarce. Therefore, the central aim of this thesis was to assess the characteristics and impact of YOD (onset $<65$ ) compared with LOD, in order to gain insight into the specific needs and care requirements of this group, which is important for the development of evidence-based guidelines for practice. Three aspects in particular were investigated: (1) the psychosocial impact of dementia on caregivers (chapter 3), (2) diagnostic issues (chapter $4 \& 5$ ) and (3) the development of neuropsychiatric symptoms during the course of the disease (chapter 6 \& 7).

We have conducted a 2-year prospective cohort study dubbed the Needs in Young-onset Dementia (NeedYD) study that obtained measurements every six months and included 215 YOD patients and their caregivers of which the results are presented in this thesis. For most of the research questions, the results obtained in this study were compared with data from an historical cohort of LOD patients, the MAAstricht Study of BEhaviour in Dementia (MAASBED) study. In the general introduction of this thesis (chapter 1) the rationale and research questions of the study are described and in chapter 2 the participants, assessment methods and procedure of the NeedYD-study are described in further detail.

To investigate whether the psychosocial impact of YOD is different from that of LOD, we conducted a literature review, which is described in chapter 3 . We hypothesized that YOD would have a higher impact on caregivers. Seventeen studies were included. The results indicated that YOD caregivers experience high levels of burden and stress. Several psychosocial problems were reported, such as a changing marital relationship, family conflict, problems with workforce participation and finances, diagnostic difficulties and a lack of designated services. Only two studies compared the impact on caregivers between YOD and LOD. No firm conclusions could be drawn based on these studies because of contradicting results and methodological limitations. However, the review did show that YOD caregivers experience high levels of psychological suffering and specific problems related to their phase in life.

In chapter 4 we investigated the time from symptom onset to diagnosis in YOD and LOD patients and attempted to determine the factors that influence this duration. The following predictors were investigated: age at onset, diagnosis, gender, education, living arrangements (alone vs not alone) and family history of dementia. We showed that it took on average 1.6 years longer from symptom onset to diagnosis in YOD patients than in LOD (2.8 versus 4.4 years). A younger age at onset and a FTD diagnosis were predictive of an extended time between symptom onset and diagnosis, whereas patients with vascular dementia received a diagnosis earlier. To obtain more information regarding the period prior to diagnosis, we analyzed qualitative interviews with 92 YOD caregivers (chapter 5). This period was commonly experienced as long and difficult. Cognitive and behavioral changes 
in the person with YOD were common and difficult to understand for caregivers. Marital difficulties were common and frequently related to behavioral changes, sometimes leading to thoughts about divorce. In addition, problems with children and issues related to work, such as early discharge or financial hardship were important topics. Based on the subjective experience of these caregivers, the following possible barriers to diagnosis were identified: misattribution of symptoms by caregivers (often psychological causes), denial of the individual with YOD and the refusal to seek medical advice, lack of confirmation from the social context (e.g. not knowing about problems at work), lack of responsiveness of the general practitioner, misdiagnosis leading and inadequate advice/help, which happened in $45 \%$ of the cases. The diagnosis burnout or depression was most frequently given prior to the dementia diagnosis. The previous chapters underline the importance of a timely diagnosis in YOD.

The next two chapters provide the first two longitudinal studies investigating the development of neuropsychiatric symptoms in YOD. We found that over the course of two years, the incidence, prevalence and persistence of neuropsychiatric symptoms was overall lower in YO-AD, with significant differences for delusions, agitation, depression, anxiety, apathy, irritability and aberrant motor behavior. The frequency of individual symptoms showed large variability. Like in LO-AD, the most frequent symptom in YO-AD was apathy. The profiles of neuropsychiatric symptoms were roughly similar between groups, but eating changes ranked higher and delusions ranked lower in YO-AD. Future studies should investigate the biopsychosocial mechanisms resulting in the strikingly lower frequency parameters of neuropsychiatric symptoms in YO-AD patients.

In chapter 7 we investigated differences in awareness between YO-AD and LO$\mathrm{AD}$ and the association with affective symptoms. Anecdotal evidence suggested that awareness levels were higher in younger patients, but no empirical studies were conducted. We were the first to demonstrate that YO-AD patients indeed had higher levels of awareness than LO-AD patients over a one-year period. High levels of awareness were associated with depression but not anxiety over the study period and this effect was stronger in the YO-AD group at baseline. A causal relationship between awareness and affective symptoms could not be established. This study showed that alertness to affective symptoms in YO-AD patients with high awareness is important and that YO-AD patients may have greater treatment benefits due to their higher awareness.

In chapter 8 , the results of this thesis are summarized, the theoretical and methodological considerations discussed and implications for future research and directions for clinical practice are addressed. 


\section{Samenvatting}

Jonge mensen met dementie worden steeds meer als een specifieke groep gezien binnen de gezondheidszorg. Deze groep heeft mogelijk andere zorgbehoeften dan de groep ouderen met dementie, maar er is nog weinig wetenschappelijk onderzoek op dit gebied gedaan. De centrale doelstelling van het onderzoek in dit proefschrift was dan ook het in kaart brengen van de klinische kenmerken en impact van dementie op jonge leeftijd (onset $<65$ jaar) en deze te vergelijken met dementie op oudere leeftijd. Deze informatie is belangrijk voor de ontwikkeling van evidence based richtlijnen voor de klinische praktijk. Drie specifieke aspecten werden onderzocht: (1) de psychosociale impact van dementie op mantelzorgers (hoofstuk 3), (2) het diagnostisch proces (hoofdstuk 4 \& 5) en (3) het ontstaan van gedragsproblemen tijdens de ziekte (hoofdstuk $6 \& 7$ ).

Om dit te onderzoeken hebben wij een prospectieve cohort-studie met een follow-up van 2 jaar uitgevoerd, de NeedYD-studie (Needs in Young onset Dementia), met 5 meetmomenten om het half jaar, waarvan de resultaten beschreven zijn in dit proefschrift. In deze studie zijn 215 jong dementerenden en hun mantelzorgers geïncludeerd. Voor de meeste onderzoeksvragen, zijn de gegevens die verkregen zijn in de NeedYD-studie vergeleken met een groep ouderen met dementie uit de MAASBED-studie (MAAstricht Study of BEhaviour in Dementia). In de algemene introductie (hoofdstuk 1) van dit proefschrift worden de rationale en vraagstellingen van het onderzoek beschreven en in hoofdstuk 2 wordt een gedetailleerd overzicht gegeven van de deelnemers, de meetmethoden en analysetechnieken die gebruikt zijn voor de studie.

Om te onderzoeken of de psychosociale impact van dementie op jonge leeftijd op mantelzorgers anders is dan die van dementie op oudere leeftijd, hebben we een systematische literatuur review uitgevoerd, die beschreven is in hoofdstuk 3 . Onze hypothese was dat de impact van dementie op jonge leeftijd op mantelzorgers hoger zou zijn. Zeventien studies werden geïncludeerd. Hieruit kwam naar voren dat mantelzorgers van jonge personen met dementie in hoge mate aan stress en depressieve symptomen lijden. Tevens werden veel psychosociale problemen gerapporteerd, zoals relatieproblemen, conflicten binnen het gezin, werkgerelateerde en financiële moeilijkheden, negatieve ervaringen m.b.t. de diagnostiek en een gebrek aan gespecialiseerde voorzieningen. Slechts 2 studies hadden als doel de impact op mantelzorgers te vergelijken tussen dementie op jonge en oudere leeftijd. Deze studies waren methodologisch beperkt en er werden tegenstrijdige resultaten gevonden. Op basis hiervan kon dan ook geen eenduidige conclusie getrokken worden. Wel werd duidelijk dat mantelzorgers van jonge personen met dementie een verhoogde lijdensdruk ervaren en dat de ziekte specifieke problemen met zich meebrengt die gerelateerd zijn aan de vroegere levensfase.

In hoofdstuk 4 is de tijd tussen het ontstaan van de eerste symptomen en diagnose en de predictoren voor deze tijdsduur vergeleken tussen jongere en oudere personen met dementie. De volgende predictoren werden onderzocht: leeftijd at onset, type dementie, geslacht, opleiding, familiaire belasting en woonsituatie 
(al dan niet alleen). Het bleek dat de duur van onset tot diagnose bij jonge mensen met dementie gemiddeld 1.6 jaar langer was dan bij oudere mensen met dementie (2.8 versus 4.4 jaar). Jongere leeftijd en frontotemporale dementie waren significante predictoren voor een langere duur tot diagnose. Vasculaire dementie bleek gerelateerd te zijn aan een kortere duur. De overige factoren bleken niet van invloed te zijn.

Om verder inzicht te verkrijgen in de periode voorafgaand aan de diagnose is een kwalitatief onderzoek uitgevoerd naar de ervaringen van mantelzorgers van jonge personen met dementie in deze periode (hoofdstuk 5). Hieruit bleek dat dit een lange en moeilijke periode was voor mantelzorgers, waarbij de diagnose soms als een opluchting werd ervaren. Cognitieve en gedragsmatige problemen kwamen veel voor bij jonge mensen met dementie en waren moeilijk te begrijpen voor mantelzorgers. Relatieproblemen werden tevens veelvuldig genoemd en meestal in verband gebracht met deze gedragsveranderingen. In enkele gevallen werd een echtscheiding overwogen voordat de diagnose werd gesteld. Daarnaast werd gerapporteerd dat kinderen tegen problemen aanliepen en dat er problemen op het gebied van werk en/of financiën ontstonden. Op basis van de subjectieve beleving van mantelzorgers, leken de volgende factoren gerelateerd te zijn aan uitstel van de diagnose: misattributie van symptomen (vaak werd gedacht aan psychische problemen), ontkenning en weigering van de jonge persoon met dementie om hulp te zoeken, gebrek aan bevestiging van de sociale context (bijvoorbeeld het niet op de hoogte zijn van problemen op het werk), gebrek aan responsiviteit van de huisarts en het krijgen van een verkeerde diagnose en inadequate zorg. Dit gebeurde in $45 \%$ van de gevallen, waarbij meestal de diagnose burnout of depressie werd gesteld voordat duidelijk werd dat er sprake was van dementie.

De volgende twee hoofdstukken beschrijven de eerste longitudinale studies gericht op het ontstaan van gedragsproblemen bij jonge personen met dementie. In de studie gepresenteerd in hoofdstuk 6 , wordt de cumulatieve prevalentie, incidentie en persistentie van gedragsproblemen onderzocht bij jonge personen met de ziekte van Alzheimer (AD) en vergeleken met oudere personen met AD tijdens de twee-jarige onderzoeksperiode. Gedragsproblemen bleken over het algemeen een lagere incidentie, prevalentie en persistentie te hebben bij jonge personen met $\mathrm{AD}$, waarbij significante verschillen werden gevonden voor wanen, agitatie, depressie, angst, apathie, prikkelbaarheid en bewegingsonrust. De frequenties van individuele symptomen lieten een hoge variabiliteit zien. Het meest voorkomende symptoom in beide groepen was apathie. Relatief gezien kwamen eetproblemen vaker voor in de jongere groep, terwijl wanen minder vaak voorkwamen dan op oudere leeftijd. Verder onderzoek zou uit moeten wijzen welke biopsychosociale mechanismen ten grondslag liggen aan de opvallend lagere frequenties van neuropsychiatrische symptomen bij jonge personen met $\mathrm{AD}$.

In hoofdstuk 7 werd ziekte-inzicht vergeleken tussen vroeg-en laat ontstane $\mathrm{AD}$ en de relatie tussen ziekte-inzicht en affectieve symptomen onderzocht. De indruk bestond dat ziekte-inzicht hoger was bij jonge mensen met dementie dan bij ouderen, maar empirische studies ontbraken. Onze studie was de eerste waarin 
werd aangetoond dat jongere personen met AD inderdaad een beter ziekte-inzicht hebben dan ouderen met AD. Intact ziekte-inzicht was geassocieerd met depressieve symptomen, maar niet met angst en dit effect was sterker in de jongere groep op baseline. Een causale relatie tussen ziekte-inzicht en affectieve symptomen kon niet vastgesteld worden. Deze studie liet zien dat alertheid op affectieve symptomen bij jonge mensen met AD met adequaat ziekte-inzicht belangrijk is en dat behandelings-en begeleidingseffecten mogelijk hoger zijn in deze groep, vanwege het hogere ziekte-inzicht.

In hoofdstuk 8 worden de resultaten samengevat, de theoretische en methodologische aspecten besproken en implicaties van de bevindingen voor de klinische praktijk en toekomstig onderzoek gegeven. 